\title{
Impurity-induced resonant Raman scattering in GaAs below the band gap at low temperature
}

\author{
Dissertation \\ zur Erlangung des Doktorgrades \\ der Mathematisch-Naturwissenschaftlichen Fakultäten \\ der Georg-August-Universität zu Göttingen
}

vorgelegt von

Qing Huang

aus Jiangsu, V.R. China

Göttingen, Juni 2000 
D7

Referent:

Prof. Dr. R. G. Ulbrich

Korreferent:

Prof. Dr. W. Felsch

Tag der mündlichen Prüfung:

21. Juni 2000 


\section{Contents}

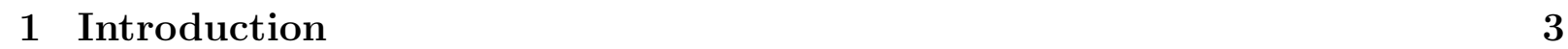

\begin{tabular}{|lll}
\hline 2 & Intrinsic first-order resonance Raman scattering in GaAs & 7
\end{tabular}

$2.1 \quad$ Lattice, electronic band structure and phonons of GaAs . . . . . . . . . . . 7

$2.2 \quad$ First-order resonance Raman scattering (RRS) . . . . . . . . . . . . . . . . 11

2.3 Electron-phonon interactions $\ldots \ldots \ldots \ldots$. . . . . . . . . . . . . . 12

2.3 .1 Interactions between electrons and acoustic phonons . . . . . . . . . 13

2.3 .2 Interactions between electrons and optical phonons . . . . . . . . . 14

2.4 Raman tensor and selection rules . . . . . . . . . . . . . . . . . . . . . 15

2.5 Absolute cross-section for first-order RRS . . . . . . . . . . . . . . . . . . . 16

2.5 .1 Expressions for uncorrelated e-h pairs . . . . . . . . . . . . . 16

2.5 .2 Expressions including the exciton effect . . . . . . . . . . . . . . . . 19

2.6 Measurement of Raman scattering cross-sections . . . . . . . . . . . . . . . 22

3 Experimental

3.1 Set-up . . . . . . . . . . . . . . . . . . . . . . . . . . . . 25

3.2 Procedure . . . . . . . . . . . . . . . . . . . . . . . . . 27

\begin{tabular}{|lll}
4 & Luminescence and absorption of the GaAs sample & 29
\end{tabular}

4.1 Photoluminescence . . . . . . . . . . . . . . . . . . . . . . . . . 29

$4.1 .1 \quad$ Free-to-bound transition $\left(e, A^{0}\right) \ldots \ldots \ldots$

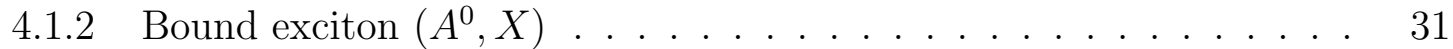

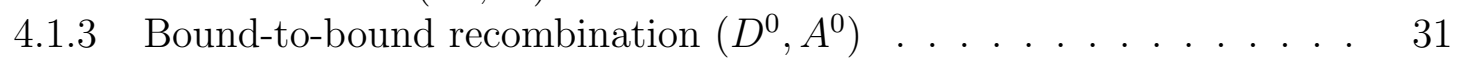

4.1 .4 Phonon replicas . . . . . . . . . . . . . . . . . . . . . . . . 32

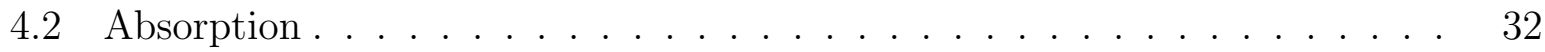

$\begin{array}{lll}5 & \text { Impurity-induced radiative transitions } & 35\end{array}$

5.1 Intrinsic transitions . . . . . . . . . . . . . . . . . . . . . 35

$5.1 .1 \quad$ Uncorrelated e-h pairs . . . . . . . . . . . . . . . . . 36

5.1 .2 Free exciton . . . . . . . . . . . . . . . . . . . . . . . . . . . . . . . . . . . 36

5.2 Extrinsic transitions $\ldots \ldots \ldots \ldots \ldots$

$5.2 .1 \quad$ Acceptor-band recombination $\left(e, A^{0}\right) \ldots \ldots \ldots$

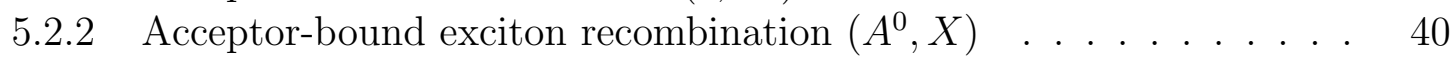


$5.2 .3 \quad$ Pair luminescence $\left(D^{0}, A^{0}\right) \ldots \ldots \ldots$. . . . . . . . . . . 43

6 Impurity-induced RRS below the $E_{0}$ band gap: results and discussions 45

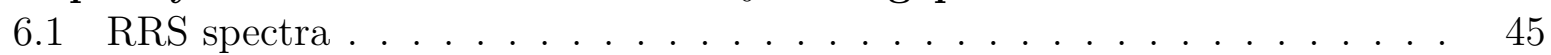

$6.2 \quad$ First-order RRS at the threshold of $\left(e, A^{0}\right) \ldots \ldots \ldots$. . . . . . . . . . 48

6.3 Cascade scattering by acoustic phonons above the $\left(e, A^{0}\right)$ threshold $\ldots . .56$

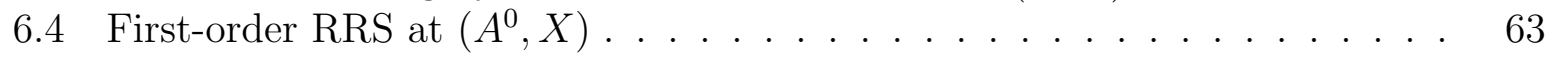

$\begin{array}{llll}7 & \text { Other spectral features due to impurity spatial distribution effect } & 67\end{array}$

7.1 Phonon replicas of luminescence $\ldots \ldots \ldots$. . . . . . . . . . . . 67

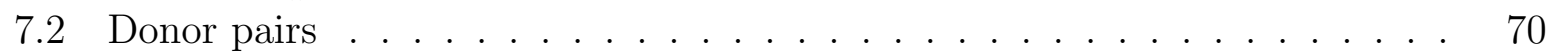

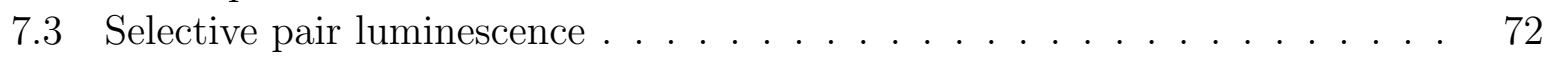

7.4 Up-converted luminescence $\ldots \ldots \ldots$. . . . . . . . . . . . . . . . . 73

$\begin{array}{lll}8 & \text { Summary } & 77\end{array}$

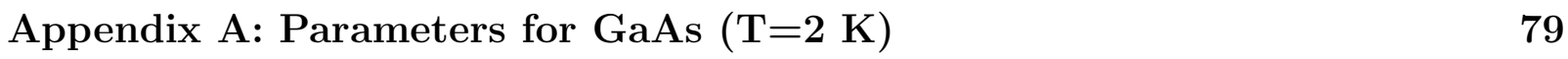

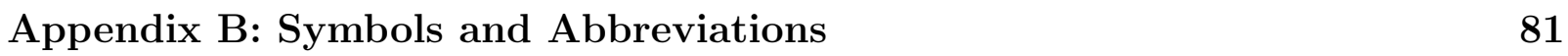

\begin{tabular}{lr}
\hline References & 84
\end{tabular} 


\section{Chapter 1}

\section{Introduction}

In 1930, Chandrasekhara Venkata Raman won Nobel Prize in Physics for the discovery of the phenomenon bearing his name: Raman scattering. Since then, the value of Raman scattering as a tool of investigating the vibrational and optical properties of gases, liquids, and solids has been fully realized. A great impetus of the application of this effect came in the 1960's due to the advent of tunable dye lasers and the availability of improved spectrometers and photon counting electronics of recording weak light. The contributions of Raman scattering as a powerful technique in many research fields are enormous. In solid state physics alone, heavy engagement in research related to light scattering phenomena has been well demonstrated in the series books [CG75-91], which have covered the most exciting and significant developments up to the beginning of the 1990's. In the last decade, as materials research is booming, the demand for understanding of structural, electrical and optical properties of new materials by Raman scattering method grows as well [Icc99.

Raman scattering is a basic experimental method in solid state physics. Particularly, when the exciting energy is resonant with electronic transitions of a studied medium, the Raman cross-sections will increase considerably. This phenomenon is known as resonance Raman scattering (RRS). RRS has proven to be very useful in probing elementary excitations and electron-phonon interactions in semiconductors.

This thesis focuses on the impurity-induced RRS below the band gap $E_{0}$ in GaAs at low temperature.

The intrinsic RRS, i.e., the RRS in ideal crystals, has been intensively studied in GaAs. Earlier, Trommer and Cardona gave a relatively thorough study in GaAs at $E_{0} / E_{0}+\Delta_{0}$, $E_{1} / E_{1}+\Delta_{1}$ [Tro78]. Grimsditch et al. measured the absolute cross-sections in the region $1.8 \mathrm{eV}$ to $2.7 \mathrm{eV}$ [Gri79]. The quantitative interpretation of the absolute cross-sections was comprehensively reviewed by a work of Cardona, where a theory based on uncorrelated electron-hole pairs was formalized [Car82. Later, Sood et al. Soo87] investigated RRS near the lowest direct absorption edge of GaAs at liquid nitrogen temperature. They found that the exciton effect should be included to give a satisfactory agreement with the experimental data. Therefore, a one-phonon RRS theory including the exciton effect has been recently developed [Can89a, Can89b, Tra89]. This theory has been applied to many III-V semiconductor compounds and can explain almost all the absolute values of cross- 
sections measured in extensive experimental studies Kau87a, Kau87b, Kau87c, Kau87d, Men85, Soo87]. These investigations of RRS concern two kinds of intrinsic scattering in terms of dipole-allowed and dipole-forbidden scattering, which are based on the following electron-phonon interaction mechanisms:

- Deformation potential (DP) and eletric-optic (EO) interaction. The latter originates from the interband Fröhlich interaction between electron-hole pairs and longitudinal optical (LO) phonons in polar crystals. These two mechanisms are responsible for the dipole-allowed scattering.

- Intraband Fröhlich interaction $(\mathrm{F})$. The scattering owing to this mechanism is $\boldsymbol{q}$ (phonon wave vector)-dependent, thus dipole-forbidden. It may become dominant near resonance.

Because we deal with the impurity-induced RRS, it is a study of extrinsic scattering processes in a disordered system. The motivation for such a study is twofold:

- For the study of intrinsic RRS at low temperature, it is required to have a correct estimate of the extrinsic contribution. It is well known that the intrinsic RRS near the band gap in direct gap semiconductors at low temperature is often masked by strong impurity luminescence. Even in high-purity materials with impurity concentration less than $10^{14} \mathrm{~cm}^{-3}$, the presence of donor and acceptor levels has profound effects on radiative recombination spectra [Ulb73]. Luminescence phonon replicas may overlap the intrinsic RRS signals seriously under some circumstances. So it is necessary to identify and extract the intrinsic RRS signals from the luminescence background.

- For the extrinsic RRS study, it is important to understand how the scattering proceeds at the impurity sites. Raman scattering is a third-order process where optical recombination processes are involved twice. At low temperature, impurity-related interband optical transitions may contribute significantly to the Raman polarizability. On the other hand, we are interested in the electron-phonon interactions, too. It is worthwhile to investigate these interactions and make it clear which role the interaction plays in the scattering. Impurity scattering involves energy and momentum transfers of electrons. So we have to clarify the energy- and momentum-relaxation of the intermediate states of impurities. In fact, impurity-induced Raman scattering has many properties different from that of intrinsic scattering. For examples, it has been verified that Raman scattering is reinforced by impurity scattering in high-purity materials like CdS [Col70, Gog76]; localized vibration modes have been found in the impurity-induced scattering [Ber87, Rey93; multi-phonon scattering has been observed in the RRS below the $E_{0}$ gap in GaAs [Roe91]; and it has been discovered that interference effects can be destroyed by extrinsic RRS near $E_{0}+\Delta_{0}$ gaps Men85, Kau87d (although it was claimed later that the intrinsic exciton effect predominated in the scattering, the contribution from impurity-induced scattering was still believed to be important under some circumstances [Can89b]). 
Additionally, a study focused on extrinsic scattering processes is also of practical value in determining the nature of impurities and optimizing the efficiency of optical components and devices.

The impurity-related optical transitions below the $E_{0}$ band gap in GaAs at low temperature are generally classified into:

- Free-to-bound recombinations, such as: $\left(e, A^{0}\right)$ and $\left(h, D^{0}\right)$.

- Bound-to-bound recombinations, such as: $\left(A^{0}, X\right),\left(D^{0}, X\right),\left(D^{+}, X\right)$, and $\left(A^{-}, X\right)$.

For the lightly-doped p-type GaAs used in this study, the recombinations of $\left(e, A^{0}\right)$ and $\left(A^{0}, X\right)$ are relatively strong at liquid helium temperature. This thesis focuses on acceptor-induced Raman scattering which has scattering resonances occurring at $\left(A^{0}, X\right)$ and at the threshold of $\left(e, A^{0}\right)$.

Carbon-doped GaAs is chosen to study impurity-induced Raman scattering, since many important parameters are well known (one can find those parameters in Refs. Ada94, Asp83, Bla82, Bog68, Soo87, Ulb73]).

To study the Raman scattering efficiencies, we make comparisons in contrast to the intrinsic scattering in such a manner:

- Scattering at the $\left(e, A^{0}\right)$ resonance $\longleftrightarrow$ scattering of free electron-hole (e-h) pairs at the $E_{0}$ gap;

- Scattering at $\left(A^{0}, X\right) \longleftrightarrow$ scattering of free 1-s excitons.

To evaluate the experimental results from the impurity-induced scattering, the thesis not only analyzes the oscillator strengths of the impurity transitions involved in the scattering, but also considers momentum transfers which lead to the breakdown of the selection rules. Both are important to understand the large scattering efficiency.

Multiple-phonon-scattering is discussed in the thesis, too. In the thesis of Rödle [Roe91] and in this work it is demonstrated that the multi-phonon scattering takes place above the threshold of $\left(e, A^{0}\right)$ and acoustic phonons participate in the scattering. This is a very interesting discovery because on one hand, the multi-phonon RRS by acoustic phonons in semiconductors was observed and reported in the past only via discrete exciton states [Kot79, Mar71a, Mar71b, Oka79, Seg68, Wil70, Yu75, Zey75, so to our knowledge, this is the first claim of scattering by dispersive acoustic phonons via the free-to-bound transition; on the other hand, it is a proof of the energy relaxation of intermediate states by emitting acoustic phonons near the minimum of conduction band. Energy relaxation of hot electrons is a fundamental process. It has been shown that cascade scattering by LO phonons is the dominant phonon-assisted relaxation mechanism when the excitation energy is much higher than the band gap. In the impurity scattering, it was predicted that "quasi-elastic" scattering by acoustic phonons could enhance the Raman scattering efficiency Gog76. Here we confirm the acoustical relaxation mechanism directly by our RRS experiment. And moreover, we show that the impurity scattering can transfer a small amount of energy by acoustic phonons with large $q$ 's, so the scattering is not "quasi-elestic" when the excitation 
is above the $\left(e, A^{0}\right)$ threshold. Therefore, the $\mathrm{RRS}$ study at the $\left(e, A^{0}\right)$ threshold can be regarded as an effective tool to trace the energy relaxation of the impurity-related intermediate states.

The thesis is arranged as follows:

After this introduction, Chapter 2 briefly describes the basic knowledge of band structure and lattice vibrational properties of GaAs, the concept of RRS, the mechanisms of electron-phonon interactions, the selection rules for intrinsic RRS and the measurement of RRS.

Chapter 3 explains the details of our experiments: the set-up and the procedure of doing the RRS experiments.

Chapter 4 investigates the impurity-induced optical transitions in GaAs at $2 \mathrm{~K}$ by recording the PL spectrum and measuring the absorption coefficient.

Chapter 5 is devoted to the quantitative description of impurity-related optical transitions in terms of oscillator strength and absorption coefficient.

Chapter 6 focuses on the analysis of the experimental results. It is divided into 4 sections: first, the RRS spectra are presented; secondly, the scattering with resonance at the threshold of $\left(e, A^{0}\right)$ is treated; thirdly, the problem of multi-phonon scattering above the threshold of $\left(e, A^{0}\right)$ is tackled; and finally, the scattering with resonance at $\left(A^{0}, X\right)$ is discussed.

Chapter 7 concerns some other spectral properties arising from impurity spatial distribution effect. Because the Raman scattering signals overlap the impurity luminescence and its phonon replicas, especially when the laser excites the impurity transitions resonantly, we have to analyze the impurity luminescence and the phonon replicas. The luminescence and its phonon replicas are dependent on both the excitation energy and the spatial distribution of the impurities.

Chapter 8 summarizes the main results of this study.

To facilitate reading, the important parameters of GaAs and the symbols and abbreviations are listed in the appendixes at the end of the thesis. Notice that the symbols and abbreviations listed in Appendix B are "globally" defined. After the explanation of the meanings of these symbols, which is given when they appear for the first time in the text, normally they may not re-explained later. Some symbols, however, are "locally" used; so these symbols are always attached with the definition explanation "in situ" and they are not be listed in Appendix B. 


\section{Chapter 2}

\section{Intrinsic first-order resonance Raman scattering in GaAs}

To understand the impurity-induced RRS in GaAs, a good knowledge of the general theory for intrinsic first-order Raman scattering is indispensable. Hence, this chapter gives an introduction of the intrinsic RRS in GaAs near the $E_{0}$ gap.

\subsection{Lattice, electronic band structure and phonons of GaAs}

GaAs is a zinc-blende-type semiconductor with the face-centered cubic (fcc) lattice. It has $T_{d}$ symmetry. The conventional cubic unit cell is illustrated in Fig. 2.1, where the primitive cell (PC) has also been plotted. The unit cube consists of four GaAs molecules in the volume of $a_{0}{ }^{3}$ ( $a_{0}$ is the lattice constant). At every lattice site there are two atoms shifted relative to each other by one quarter of the body diagonal along the [111] direction. The reciprocal lattice is body-centered cubic (bcc) and its first Brillouin zone (BZ) is drawn in Fig. 2.2, where $\Gamma, \mathrm{X}, \mathrm{L}, \mathrm{W}$, and $\mathrm{K}$ are the high symmetry points in wave vector space ( $\boldsymbol{k}$-space) at zone center and boundary, respectively. The high symmetry axes are denoted as $\Delta, \Lambda$ and $\Sigma$, respectively. These axes are used in short form to describe both phonon and electron band structures in semiconductors.

The electronic band structure of GaAs is shown in Fig. 2.3. It exhibits four empty conduction bands as well as three of four valence bands which are occupied by eight valence electrons in the $\mathrm{PC}$. The lowest conduction band has the energy minimum at the $\Gamma$ point, hence GaAs is a direct-band semiconductor. The transitions near the band gap along some high symmetry directions have been noted in the figure. This band structure was obtained by applying the $\boldsymbol{k} \cdot \boldsymbol{p}$ perturbation method at the $\Gamma$ point [Pol66]. According to the Bloch theorem, the wave functions in the reduced zone scheme are

$$
\psi_{n \boldsymbol{k}}=\exp (i \boldsymbol{k} \cdot \boldsymbol{r}) u_{n \boldsymbol{k}}(\boldsymbol{r})
$$




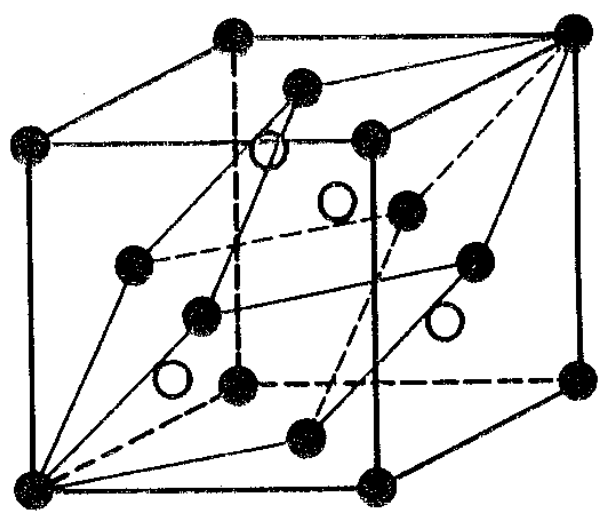

Figure 2.1: Unit cell of GaAs.

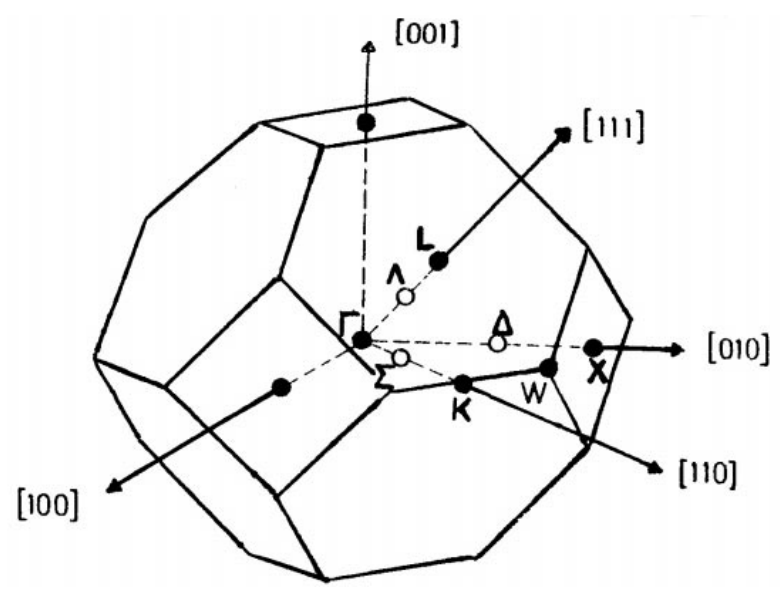

Figure 2.2: Brilloin zone of GaAs.

where $u_{n \boldsymbol{k}}$ is the periodic part of the wave functions with $n$ the band index and $\boldsymbol{k}$ lying within the first BZ.

Conventionally, the eigen-energies around band extrema can be approximated in terms of effective mass $m^{*}$ as

$$
E_{n \boldsymbol{k}}=E_{n 0}+\frac{\hbar^{2} k^{2}}{2 m^{*}}
$$

For electrons, $m^{*}$ is more or less constant near the center of the BZ, therefore Eq. 2.2 gives a parabolic dispersion relation at the $\Gamma$ point. However, for holes $m^{*}$ is very anisotropic. In the following we denote $m_{e}$ and $m_{h}$ as electron effective mass and hole effective mass, respectively.

With the consideration of spin-orbit coupling, the symmetry of the eigen-wave-functions $u_{n \boldsymbol{k}}$, namely $\left|J, m_{J}\right\rangle$ ( $J$ is the total angular momentum), are given by [Kan56]

c: $\quad|i S \uparrow\rangle,|i S \uparrow\rangle$ 


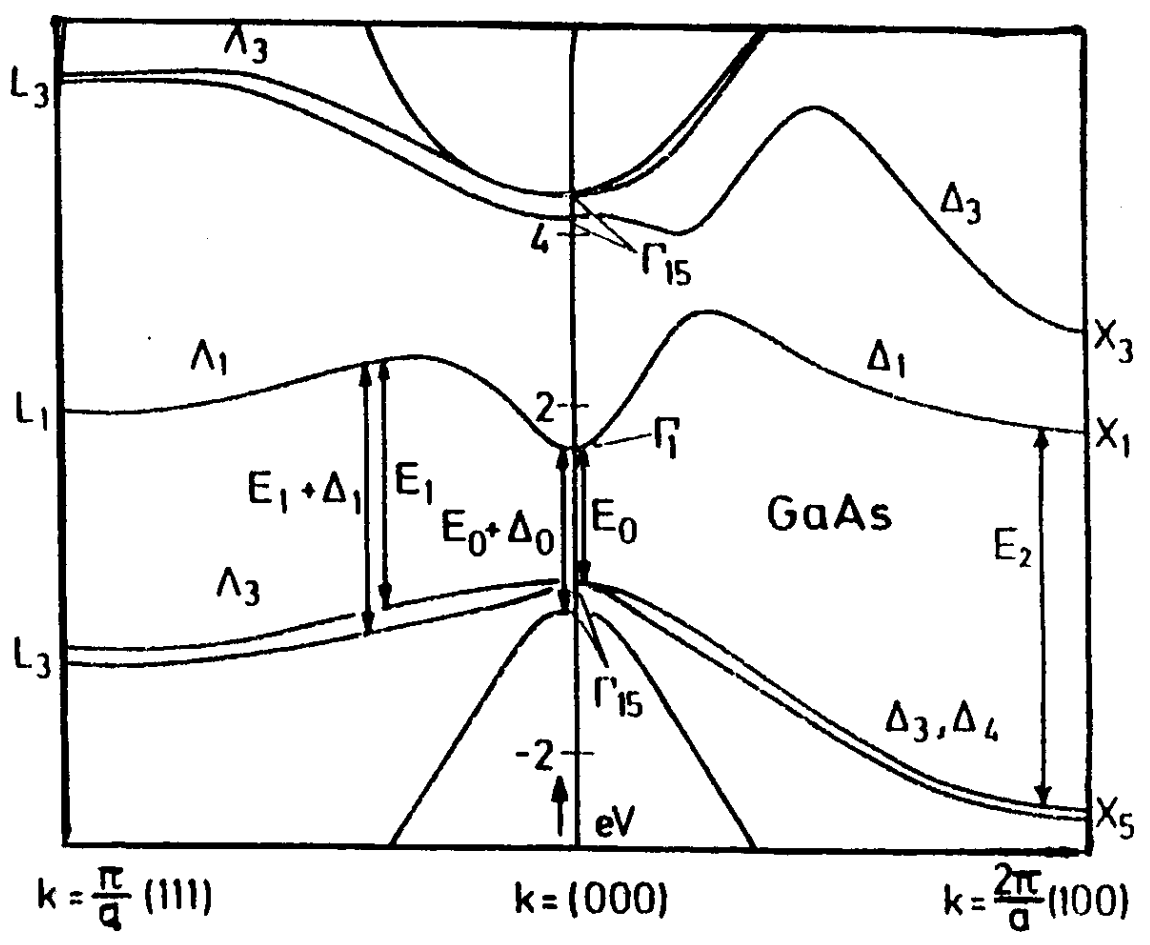

Figure 2.3: Band structure of GaAs [Pol66].

hh: $\quad|3 / 2,3 / 2\rangle=|(x+i y) \uparrow\rangle / \sqrt{2}$

$|3 / 2,-3 / 2\rangle=|(x-i y) \downarrow\rangle / \sqrt{2}$

lh: $\quad|3 / 2,1 / 2\rangle=|(-x-i y) \downarrow+2 z \uparrow\rangle / \sqrt{6}$

$|3 / 2,-1 / 2\rangle=|(x-i y) \uparrow+2 z \downarrow\rangle / \sqrt{6}$

so:

$|1 / 2,1 / 2\rangle=|(-x-i y) \downarrow-z \uparrow\rangle / \sqrt{3}$

$|1 / 2,-1 / 2\rangle=|(x-i y) \uparrow-z \downarrow\rangle / \sqrt{3}$

Here $c, h h, l h$, and so stand for conduction electron, heavy hole, light hole, spin-orbit splitting bands, respectively. $\uparrow$ and $\downarrow$ distinguish both spin orientations: spin-up and spindown. The electron is $s$-type in conduction band while the holes are $p$-like in valence bands. $x, y, z$ are customarily representing the three basis wave functions. According to group theory, the $s$-type state has $\Gamma_{1}$ symmetry whereas the $p$-type state has $\Gamma_{15}$ symmetry at $\Gamma$ point in the zinc-blende structure. The optical coupling between $\Gamma_{1}$ and $\Gamma_{15}$ states is dipole-allowed.

The lattice dynamics of GaAs has also been systematically studied [Str90, Wau63]. Fig. 2.4 shows the phonon dispersion curves along the high symmetry axes, where the experimental points were measured at $12 \mathrm{~K}$ by neutron scattering and the continuous lines were calculated with a 15-parameter rigid-ion model.

Corresponding to the two atoms per PC, there are six phonon branches: three acoustic 


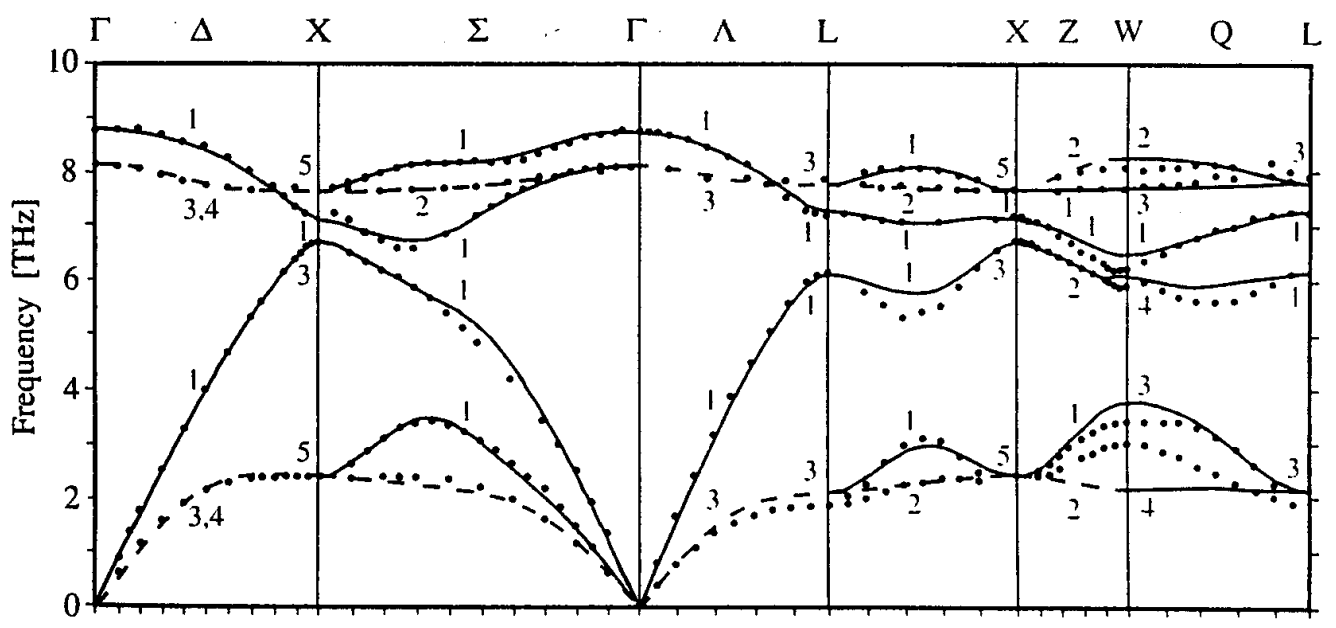

Figure 2.4: Phonon dispersion curves in GaAs along high-symmetry axes ( $\mathrm{T}=12 \mathrm{~K}$ ) [Str90]. The numbers next to the phonon branches label the corresponding irreducible representations.

phonon branches and three optical phonon branches. For the optical phonons, the different atoms in the $\mathrm{PC}$ move in different directions; whereas for the acoustic phonons, the different atoms in the $\mathrm{PC}$ move in the same direction. The difference between acoustic phonons and optical phonons can also be distinguished in their vibration frequency properties: at $\boldsymbol{q}=\mathbf{0}$, ( $\boldsymbol{q}$ denotes phonon wave vector), the frequencies of acoustic phonons are zero while the frequencies of optical phonon are nonvanishing.

Along the high symmetry directions, we can further classify these phonon branches by considering the relationship between the direction of phonon polarization $\boldsymbol{p}_{0}$ and the direction of phonon propagation. If the direction of phonon polarization is perpendicular to the direction of phonon propagation, the phonon is called transverse phonon; if the two directions are parallel to each other, the phonon is called longitudinal phonon. These differently defined vibrational modes are schematically illustrated by a linear diatomic chain in Fig. 2.5 .

Taking GaAs as an example, one observes that along [100] direction, there is one longitudinal acoustic (LA) phonon branch and two degenerate transverse acoustic phonon (TA) branches, and similarly, there are one longitudinal optical (LO) phonon branches and two degenerate transverse optical phonon (TO) branches, as shown in Fig. 2.4.

For wave vectors near the center of the BZ, the LO phonon frequency in GaAs is higher than the TO phonon frequencies due to the partially ionic nature of the bonding between Ga and As atoms. The frequency ratio between LO and TO phonons is described by the Lyddane-Sachs-Teller relation as

$$
\frac{\omega_{L O}^{2}}{\omega_{T O}^{2}}=\frac{\epsilon_{0}}{\epsilon_{\infty}}
$$

where $\epsilon_{0}$ and $\epsilon_{\infty}$ are static and optical dielectric constants, respectively.

Besides the essential difference of frequency properties for acoustic and optical phonons 


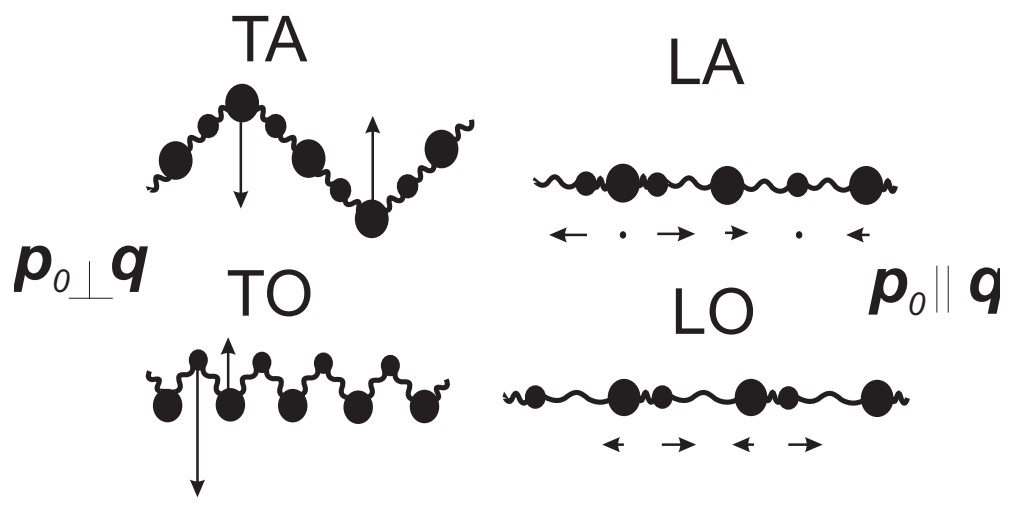

Figure 2.5: Different vibrational modes of a linear diatomic chain. $\boldsymbol{p}_{0}$ and $\boldsymbol{q}$ represent the polarization and propagation direction of the phonons. The arrows indicate the movements of the atoms. Meanings of letters: L-longitudinal, T-transverse, A-acoustic (phonon), O-optical (phonon).

near the $\Gamma$ point, we would like to emphasize here another primary frequency distinction: the frequencies of optical phonons change little within the BZ; on the contrary, the frequencies of acoustic phonons show a nearly linear dependence of wave number near the BZ center. This acoustical dispersion relation can help us identify the participation of acoustic phonons in the impurity-induced multi-phonon scattering in this study, as will be shown in Chapter 6.

\section{$2.2 \quad$ First-order resonance Raman scattering (RRS)}

This section discusses the case where only one phonon takes part in the scattering.

Raman scattering is inelastic scattering with the frequencies of the scattering light shifted with respect to that of the incident light: the shift to lower energy side is called Stokes shift while the shift to higher energy side is called anti-Stokes shift. Besides the frequency change, accordingly, the momenta of light wave vectors alter in the scattering, too. Due to the quasi-momentum conservation rule in the crystalline solid, the change of momenta of light wave vectors must be compensated by wave vectors of involved phonons in the scattering process. However, this momentum of light in the visible region is only of the order $10^{4} \mathrm{~cm}^{-1}$, rather small compared to the size of the BZ which has a value of $10^{8} \mathrm{~cm}^{-1}$ at the boundary in GaAs. Therefore the phonons we are dealing with are long wavelength phonons, whose wave number $q$ can be justifiably approximated as $q \simeq 0$.

Resonance Raman scattering (RRS) takes place when the exciting photon energy is close to the interband transitions in the medium. Under resonant excitation condition, the scattering efficiency is strongly enhanced. In semiconductors, such a scattering process can be described microscopically with the aid of the Feynman diagram technique. There are six Feynman diagrams representing the Raman scattering. Fig. 2.6 shows one of the 
diagrams, which corresponds to the most dominant contribution for the case of resonance scattering. It describes a third-order perturbation process: an incident photon produces at first an electron-hole (e-h) pair in the semiconductor; then, the electron is scattered by one phonon, losing part of the energy to the phonon; finally, the electron recombines with the hole, emitting a photon with frequency less than that of the incident photon. In the figure, $H_{E R}$ and $H_{E P}$ represent the coupling strength of photons (radiation) with electrons, and electrons with phonons, respectively.

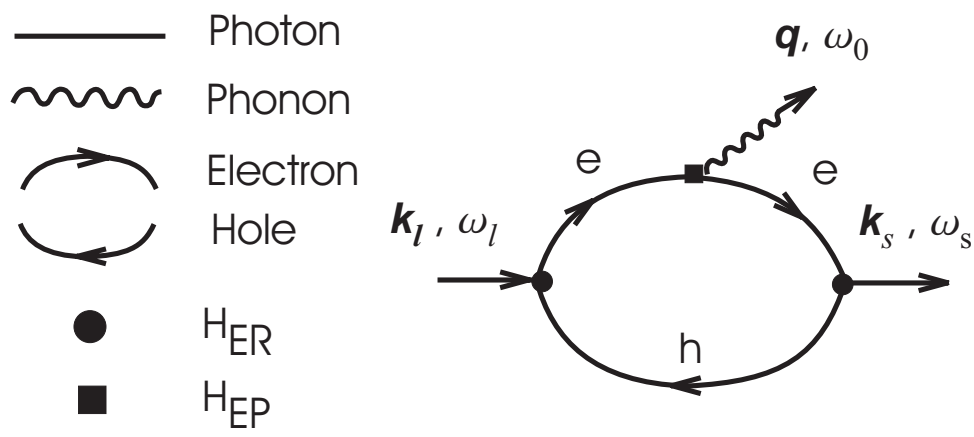

Figure 2.6: Feynman diagram for one dominant first-order Raman scattering process.

Equivalent to this diagrammatic description, the scattering probability given by Fermi's Golden Rule in third-order perturbation theory is [Lou64]

$$
R_{f i}=\frac{2 \pi}{\hbar}\left|\sum_{\alpha, \beta} \frac{\left\langle f\left|H_{E R}\right| \beta\right\rangle\left\langle\beta\left|H_{E P}\right| \alpha\right\rangle\left\langle\alpha\left|H_{E R}\right| i\right\rangle}{\left(\hbar \omega_{l}-E_{\beta}+i \Gamma_{\beta}\right)\left(\hbar \omega_{s}-E_{\alpha}+i \Gamma_{\alpha}\right)}+C\right|^{2} \cdot \delta\left(\hbar \omega_{l}-\hbar \omega_{s}-\hbar \omega_{0}\right)
$$

where $i$ stands for an initial state, $f$ stands for a final state and $\alpha$ and $\beta$ are intermediate states; $\omega_{0}$ is the phonon frequency. On the right hand side (rhs) of the Eq. 2.4 we have already included the less resonant terms, which result from the other five Feynman microscopic processes, by lumping them into a constant C. Notice that the denominator contains a product of $\left(E_{\alpha}-\hbar \omega_{l}\right)$ and $\left(E_{\alpha}-\hbar \omega_{s}\right)$. So a scattering enhancement will be exhibited when $\omega_{l}$ is in the vicinity of either $E_{\beta}$ or $E_{\alpha}+\hbar \omega_{0}$. The $\left(E_{\beta}-\hbar \omega_{l}\right)$ is referred to as an incoming resonance while $\left(E_{\alpha}-\hbar \omega_{s}\right)$ is an outgoing resonance. $\Gamma_{\alpha(\beta)}$ are the damping constants.

\subsection{Electron-phonon interactions}

As shown in Eq. 2.4, to investigate the Raman scattering one has to know the mechanisms of electron-phonon interactions $H_{E P}$. Here we refer to the book [Yu99] for a survey of these mechanisms. 
Under adiabatic approximation (i.e., Born-Oppenheimer approximation) the electronphonon interaction Hamiltonian can be expressed as the first term of a Taylor series expansion:

$$
\left.H_{E P}\left(\boldsymbol{r}_{i}, \delta \boldsymbol{R}_{j}\right) \approx \sum_{j}\left(\frac{\partial E_{n}}{\partial \boldsymbol{R}_{j}}\right)\right|_{\boldsymbol{R}_{j 0}} \cdot \delta \boldsymbol{R}_{j} \quad,
$$

where the displacement $\delta \boldsymbol{R}_{j}$, in terms of phonon creation and annihilation operators $c_{\boldsymbol{q}}^{+}$ and $c_{\boldsymbol{q}}$, can be expressed as

$$
\delta \boldsymbol{R}_{j}=\sum_{\boldsymbol{q}}\left(\frac{\hbar}{2 N v_{0} \rho \omega_{0}}\right)^{1 / 2} \hat{\mathbf{e}}_{q}\left\{c_{q}^{+} \exp \left[i\left(\boldsymbol{q} \cdot \boldsymbol{r}_{j}-\omega_{0} t\right)\right]+c . c .\right\}
$$

where $N$ is the number of unit cells in the crystal, $v_{0}$ and $\rho$ are, respectively, the volume of the $\mathrm{PC}$ and the mass density of the crystal; $\hat{\mathbf{e}}_{q}$ is the phonon polarization unit vector, and c.c. stands for the complex conjugate term.

In the following the interaction mechanisms for acoustic phonons and for optical phonons will be discussed separately.

\subsubsection{Interactions between electrons and acoustic phonons Acoustical deformation potential interaction}

The atomic displacement can change the potential which electrons feel, thus change the electronic energies. This energy change caused by lattice distortion is called deformation potential (DP). For GaAs, the volume deformation potential is the main mechanism for scattering by a LA phonon. The interaction can be described as

$$
H_{D P-V A}=d_{V A}(\boldsymbol{q} \cdot \delta \boldsymbol{R}),
$$

where $d_{V A}$ is known as the volume deformation potential, and $\delta \boldsymbol{R}$ is defined as in Eq. 2.6.

$H_{D P-V A}$ is a short-range interaction. From Eq. 2.7, one sees that $H_{D P-V A}$ depends on $q$ explicitly as $q^{1 / 2}$.

\section{Piezoelectric interaction}

For noncentrosymmetric polar crystals, lattice acoustical movement can induce a strain, with which a macroscopic polarization field $E$ is accompanied. This induced field can change the electronic energies, too. So it gives rise to the so-called piezoelectric interaction $(\mathrm{PE})$ between electron and acoustic phonons $H_{P E}$ :

$$
H_{P E}=\frac{|e|}{q^{2} \epsilon_{\infty}} \boldsymbol{q} \cdot \mathbf{e}_{\mathbf{m}} \cdot(\boldsymbol{q} \delta \boldsymbol{R}),
$$

where $\mathbf{e}_{\mathbf{m}}$ is a third-rank electromechanical tensor. 
Compared with Eq. 2.7. Eq. 2.8 contains additional $(1 / q)$ dependence. Thus piezoelectric interaction is long-range effective. In GaAs, the TA phonon scattering mainly arises from this interaction mechanism.

\subsubsection{Interactions between electrons and optical phonons Optical deformation potential interaction}

A long-wavelength optical phonon results in atomic displacement, too. However, this sort of displacement occurs only within a PC, with two or more atoms moving to each other relatively. Therefore, unlike acoustic phonons, a long-wavelength optical phonon does not induce macroscopic distortion in the crystal. There is only microscopic distortions within the PC.

For such microscopic distortions, the electronic energies can also be changed. The interaction due to optical deformation potential can be described as

$$
H_{D P-O}=D_{n \boldsymbol{k}}\left(u / a_{0}\right),
$$

where $D_{n \boldsymbol{k}}$ is defined as optical phonon deformation potential for the energy band indexed by $n$ and $\boldsymbol{k}$.

As seen from Eq. 2.9, this deformation potential interaction does not depend on the phonon wave vector. It is a short-range interaction.

\section{Fröhlich interaction}

Similar to the case for acoustic phonons, a long-wavelength LO phonon in polar crystal generates a macroscopic electric field, too. This electron-LO phonon interaction is called Fröhlich interaction and it is a long-range interaction.

The Fröhlich Hamiltonian is

$$
H_{F}=\sum_{\boldsymbol{q}} \frac{i C_{F} / q}{\sqrt{N v_{0}}}\left\{c_{q}^{+} \exp \left[i\left(\boldsymbol{q} \cdot \boldsymbol{r}-\omega_{L O} t\right)\right]-c . c .\right\}
$$

where the coefficient $C_{F}$ is given by

$$
C_{F}=e\left[2 \pi \hbar \omega_{L O}\left(\epsilon_{\infty}^{-1}-\epsilon_{0}^{-1}\right)\right]^{1 / 2}
$$

The evaluation of Fröhlich interaction between electronic states $i$ and $j$ can be expressed in terms of matrix elements as

$$
\left\langle(n+1), i\left|H_{F}\right| n, j\right\rangle \propto C_{F}\left[\frac{\delta_{i j}}{|q|}+\langle i|\boldsymbol{q} \cdot \boldsymbol{p}| j\rangle\left(1-\delta_{i j}\right)\right] .
$$

Two terms appear in Eq. 2.12, which correspond to two mechanisms contributing the scattering efficiency: 
- The first term leads to the intraband Fröhlich (F) scattering since it is nonvanishing only if $i$ and $j$ belong to the same energy band.

- The second term contributes to the interband scattering because it is nonzero only if $i$ and $j$ belong to different bands. It arises from wave functions under $\boldsymbol{k} \cdot \boldsymbol{p}$ perturbation consideration. It is also called electro-optic (EO) scattering because the coupling of different bands is mediated via the electric field of the scattered LO phonon.

\subsection{Raman tensor and selection rules}

The Raman tensor $\mathbf{R}$ is a second-rank tensor which is introduced to evaluate the symmetry of scattered phonon and the intensity of scattered radiation. The Raman scattering intensity is obtained from

$$
I_{s} \propto\left|\hat{\mathbf{e}}_{l} \cdot \mathbf{R} \cdot \hat{\mathbf{e}}_{s}\right|^{2},
$$

where $\hat{\mathbf{e}}_{l(s)}$ is the polarization vector of the incident (scattered) light.

By inspecting electron-phonon interactions, polarizations of incident and scattered light, and symmetries of scattered phonons, one can deduce the symmetry of Raman tensor, i.e., the selection rules for Raman scattering.

Hayes and Loudon have tabulated the selection rules for all kinds of crystals Hay78, Lou64. The derived selection rules hold solely for long wavelength optical phonons $(q \simeq 0)$, because only in this case the symmetries can be analyzed by simply applying point group theory. For GaAs, the crystal has $T_{d}$ symmetry, correspondingly, and the Raman tensor for the $\Gamma_{15}$ phonon is given by

$$
\mathbf{R}(X)=\left(\begin{array}{ccc}
0 & 0 & 0 \\
0 & 0 & a_{1} \\
0 & a_{1} & 0
\end{array}\right), \mathbf{R}(Y)=\left(\begin{array}{ccc}
0 & 0 & a_{1} \\
0 & 0 & 0 \\
a_{1} & 0 & 0
\end{array}\right), \mathbf{R}(Z)=\left(\begin{array}{ccc}
0 & a_{1} & 0 \\
a_{1} & 0 & 0 \\
0 & 0 & 0
\end{array}\right)
$$

Here we choose crystal coordinations, with $X, Y$ and $Z$ defined as [100], [010] and [001] directions, respectively. The $X, Y$ and $Z$ in the brackets show the polarization directions of scattered phonons. The Raman tensor in such a form is due to the deformation potential, and, if in polar crystals, also the electric-optic electron-phonon interaction (interband Fröhlich). According to Eq. 2.14, the selection rules for back-scattering from the (100) surface of GaAs is obtained in Table 2.1.

For intraband Fröhlich scattering, the Raman tensor obeys new selection rules. Because the scattering is intraband, it immediately follows that $R_{f i}$ in Eq. 2.4 is large only for $\alpha=\beta$, i.e., only diagonal $\left(\hat{\mathbf{e}}_{l} \| \hat{\mathbf{e}}_{s}\right)$ scattering is nonvanishing in this order [Mar71b]. The Raman tensor can be expressed as

$$
\mathbf{R}(X)=\left(\begin{array}{ccc}
a_{2} & 0 & 0 \\
0 & a_{2} & 0 \\
0 & 0 & a_{2}
\end{array}\right)
$$


Table 2.1: Selection rules for allowed Raman scattering

\begin{tabular}{c|c|c}
\hline \hline$\hat{\mathbf{e}}_{l}$ & $\hat{\mathbf{e}}_{s}$ & symmetry component \\
\hline$[100]$ & {$[100]$} & $\Gamma_{1}, \Gamma_{3}$ \\
\hline$[100]$ & {$[010]$} & $\Gamma_{15}(\mathrm{LO})$ \\
\hline$[110]$ & {$[110]$} & $\Gamma_{1}, \Gamma_{3}, \Gamma_{15}(\mathrm{LO})$ \\
\hline$[110]$ & {$[110]$} & $\Gamma_{3}$ \\
\hline
\end{tabular}

Since the Raman tensor in Eq. 2.15 is diagonal, the intraband scattering can be observed in parallel configurations in the back-scattering geometry. Further analysis shows that the F-induced scattering is $\boldsymbol{q}$-dependent: the scattering intensity is proportional to $q^{2}$ Mar71b. In contrast to the DP- or EO- induced Raman scattering where the scattering is allowed for long wavelength phonons, the intraband F-induced scattering approaches vanishing when the wave vector of scattered phonon is taken to be zero. Therefore, in the dipole approximation the intraband F-induced scattering is also called forbidden scattering.

\subsection{Absolute cross-section for first-order RRS}

\subsubsection{Expressions for uncorrelated e-h pairs}

Cardona has derived the explicit expression of Raman polarizability for uncorrelated e-h pairs in semiconductors [Car82]. In his formalism, the Raman polarizability $\chi_{s}$ is obtained based on the relationship that it is the derivative of the standard linear polarizability $\chi$ with respect to the normal coordinates $\zeta$, i.e.,

$$
\chi_{s}(\omega)=\frac{\chi\left(\omega_{l}\right)-\chi\left(\omega_{s}\right)}{\omega_{0}} \frac{d \omega_{0}}{d \zeta}(n+1)^{1 / 2} \sqrt{\frac{1}{2 \omega_{0}}}+C,
$$

where $n$ is the phonon number and $C$ is a constant.

The differential cross section of Stokes scattering, derived from second-order perturbation calculation with damping neglected, has the form:

$$
\begin{aligned}
\frac{\partial^{2} \sigma_{s}}{\partial \omega_{s} \Omega}= & \frac{\omega_{s}^{2}}{\omega_{l}^{2}} r_{e}^{2}\left[\sum_{i, n_{i}}<0, n_{f}\left|e^{-i \boldsymbol{k}_{s} \cdot \boldsymbol{r}} \hat{\mathbf{e}}_{s} \cdot\left(\boldsymbol{p}-\boldsymbol{k}_{s} / 2\right)\right| i, n_{i}><0, n_{i}\left|e^{i \boldsymbol{k}_{l} \cdot \boldsymbol{r}} \hat{\mathbf{e}}_{l} \cdot\left(\boldsymbol{p}+\boldsymbol{k}_{l} / 2\right)\right| i, n_{0}>\right. \\
& \left.\frac{1}{\omega_{i, n_{i}}-\omega_{0, n_{0}}-\omega_{l}}+C\right]^{2} \delta\left(\omega_{i, n_{f}}-\omega_{0, n_{0}}-\omega_{0}\right),
\end{aligned}
$$

where $r_{e}=2.8 \times 10^{-15} \mathrm{~m}^{2}$, called classical electron radius; $n_{(0, i, f)}$ here denotes the vibrational part of the wave function; $\boldsymbol{p}$ is the momentum operator of the electron and $\boldsymbol{k}_{l(s)}$ is wave vector of incident (scattered) light; $C$ represents the non-resonant term for scattering. 
In dipole approximation, $\boldsymbol{k}_{l(s)} \simeq 0$; so the matrix element can be simplified by calculating $\boldsymbol{p}$ alone, which is taken as $\boldsymbol{p}_{c v}$ for the e-h pairs in conduction and valence bands.

By neglecting the vibrational structure, taking $v \rightarrow c$ excitations for the intermediate states, the corresponding linear polarizability near the $E_{0}$ band gap is derived from Eq. 2.17] as

$$
\chi \approx \frac{4 \pi V_{g}^{-1}}{\omega_{l} \omega_{s}}\left[\sum_{i, n_{i}}<0, n_{0}+1\left|\boldsymbol{p}_{c v}\right| i, n_{i}><0, n_{i}\left|\boldsymbol{p}_{c v}\right| i, n_{0}>\frac{1}{\omega_{i, n_{i}}-\omega_{0, n_{0}}-\omega_{l}}+C\right]
$$

where $V_{g}$ is the volume of the crystal and $C$ is a constant.

Transforming the sum over $i$ in Eq. 2.18 into an integral over $\omega$ leads to

$$
\chi(\omega)=4 \pi \int_{0}^{\infty} \frac{\boldsymbol{p}_{c v} \times \boldsymbol{p}_{c v}}{\omega^{\prime 2}} N_{d}\left(\omega^{\prime}\right) \frac{1}{\omega^{\prime}-\omega} d \omega,
$$

where $N_{d}(\omega)=\frac{2^{1 / 2}}{\hbar \pi^{2}} \mu^{3 / 2}\left(\hbar \omega-E_{0}\right)^{1 / 2}$ for $\hbar \omega>E_{0}$, is the combined density of states near a 3-dimensional critical point, with $\mu$ as the reduced effective mass of an e-h pair (or, exciton effective mass), which is given by $\mu^{-1}=m_{e}^{-1}+m_{h}^{-1}$.

The integration of Eq. 2.19 yields the linear polarizability function $\chi(\omega)$ :

$$
\chi(\omega)=F_{0} F(x),
$$

with $F_{0}$ a constant and $F(x)$ defined as

$$
F(x)=x^{-2}\left[2-(1+x)^{1 / 2}-(1-x)^{1 / 2}\right],
$$

where $x$ is reduced energy, defined as $x=\hbar \omega / E_{0}$. The real and imaginary part of $F(x)$ is plotted in Fig. 2.7.

Due to the relationship between the Raman polarizablity $\chi_{s}$ and the linear polarizablity $\chi$ given by Eq. 2.16, one finds that

$$
\chi_{s}(\omega)=\frac{F_{0}}{2 E_{0}} G(x) \frac{d E_{0}}{d \zeta}(n+1)^{1 / 2}\left(2 \omega_{0}\right)^{-1 / 2},
$$

with

$$
G(x)=x^{-2}\left[2-(1+x)^{-1 / 2}-(1-x)^{-1 / 2}\right] .
$$

Notice that the Eq. 2.22 stands only for the two-band term for Raman polarizability. The analysis of the function $G(x)$, i.e., its real, imaginary and absolute values, is shown in Fig. 2.8. It is seen clearly that a strong scattering resonance occurs at $E_{0}$.

An more strict treatment should include the three-band term, giving additional contribution proportional to $F(x)$. So a final form of the Raman polarizability of DP-induced scattering is given by Gri79, Soo87. 


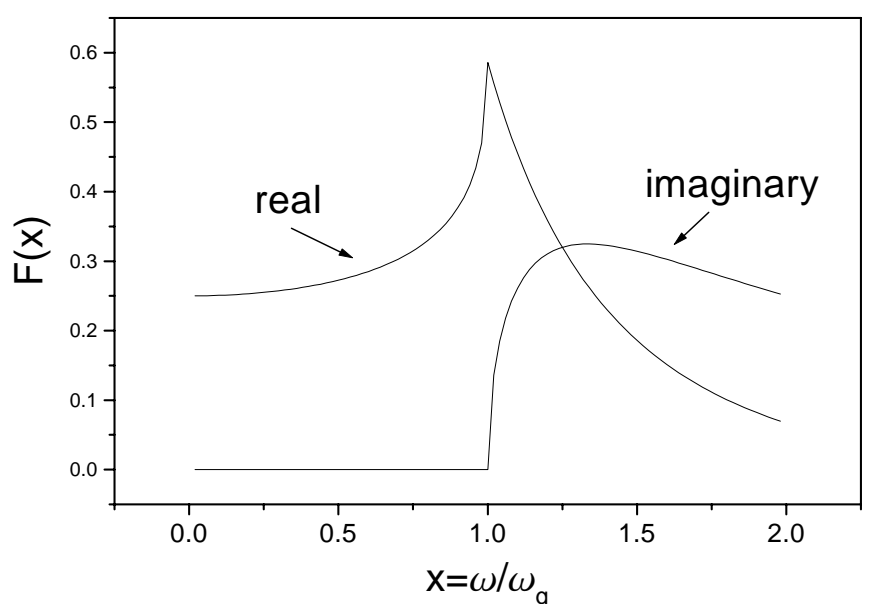

Figure 2.7: Dependence of the linear polarizability function $F(x)$ on reduced frequency.

$$
\begin{aligned}
a_{D P} & =A_{1}\left\{-G\left(x_{0}\right)+\frac{4 E_{0}}{\Delta_{0}}\left[F\left(x_{0}\right)-\left[\frac{E_{0}}{E_{0}+\Delta_{0}}\right]^{3 / 2} F\left(x_{0 s}\right)\right]\right\} \\
& +A_{2}\left[\frac{1}{1-x_{1}^{2}}+\left[\frac{E_{1}}{E_{1}+\Delta_{1}}\right]^{2} \frac{1}{1-x_{1 s}^{2}}\right]+A_{3}
\end{aligned}
$$

where $x^{\prime} s$ stand for reduced energies, defined as: $x_{0}=\hbar \omega_{l} / E_{0}, x_{o s}=\hbar \omega_{l} / E_{0}+\Delta_{0}$, $x_{1}=\hbar \omega_{l} / E_{1} x_{1 s}=\hbar \omega_{l} / E_{1}+\Delta_{1} . E_{0(1)}$ and $\Delta_{0(1)}$ are the band-band transition energies and spin-orbit splittings around critical points, respectively (see Fig. 2.3).

At $E_{0}$ resonance, $a_{D P}$ can be simplified as

$$
a_{D P} \simeq A_{1}\left\{\frac{2 E_{0}\left[F\left(x_{0}\right)-F\left(x_{0}+\hbar \omega_{T O}\right)\right]}{\hbar \omega_{T O}}+\frac{4 E_{0}}{\Delta_{0}}\left[F\left(x_{0}\right)-\left[\frac{E_{0}}{E_{0}+\Delta_{0}}\right]^{3 / 2} F\left(x_{0 s}\right)\right]\right\} .
$$

For the dipole-allowed LO scattering, the EO-interaction also contributes to the Raman scattering. The $(\mathrm{EO}+\mathrm{DP})$-induced LO scattering relates to the DP-induced TO scattering by so-called Faust-Henry coefficient F.H., which is defined as

$$
\text { F.H. }=\frac{\omega_{L O}^{2} / \omega_{T O}^{2}-1}{1-a_{L O} / a_{T O}}
$$

Hence, using F.H. $a_{L O}$ can be obtained from $a_{T O}$ (or, $a_{D P}$ ) directly; and vice versa.

Similarly, for the forbidden scattering owing to the intraband Fröhlich interaction, the Raman polarizability is derived using linear polarizabilty as [Car82, Men85] 


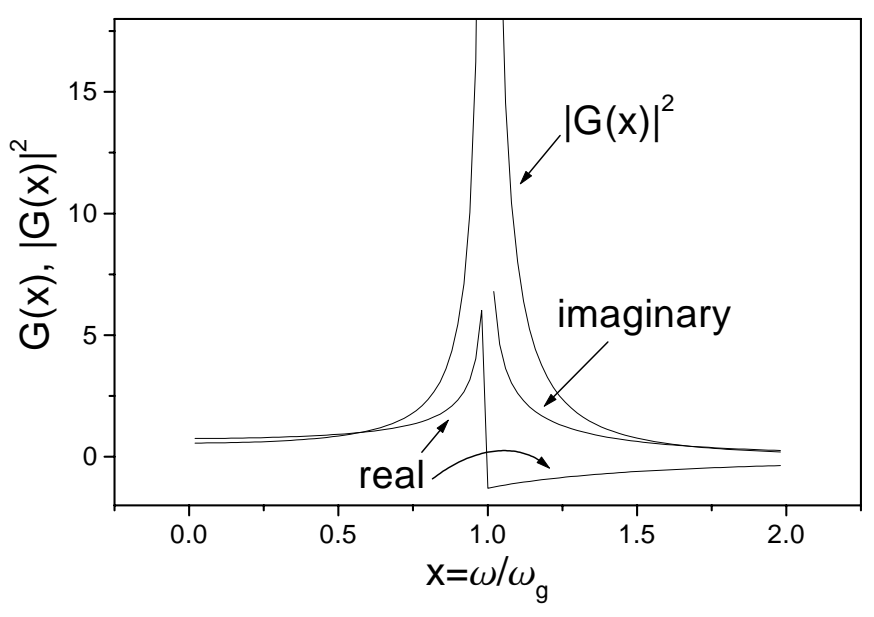

Figure 2.8: Dependence of the two-band Raman polarizability function $G(x)$ on reduced frequency.

$$
a_{F}=\frac{q}{12 \pi}\left[\frac{e}{m \hbar}\right]^{2} \frac{C_{F}}{\hbar \omega_{L O}}\left[\frac{1}{\omega_{l}}\right]^{2}\left[\frac{\omega_{l}}{\omega_{s}}\right]^{1 / 2}\left(4 v_{0} \mu M\right)^{1 / 2}\left[\frac{2 p_{c v}^{2}}{3}\right]\left(s_{e}-s_{h}\right) H\left(\omega_{l}\right),
$$

where

$$
H\left(\omega_{l}\right)=\left[\left[\frac{\hbar \omega_{l}-E_{0}+i \Gamma}{\hbar \omega_{L O}}\right]^{1 / 2}-\left[\frac{\hbar \omega_{s}-E_{0}+i \Gamma}{\hbar \omega_{L O}}\right]^{1 / 2}\right]^{3},
$$

and $s_{e, h}=m_{e, h} /\left(m_{e}+m_{h}\right)$.

The real, imaginary and absolute values of F-induced Raman polarizability function $H(x)$ are plotted in Fig. 2.9. Resonance is shown to take place at $E_{0}$.

\subsubsection{Expressions including the exciton effect}

In the section 2.5.1, the excition effect, i.e., the Coulomb interaction between e-h pairs is not taken into account in the calculation. However, the exciton effect can enhance scattering efficiencies in many III-V compounds, especially at low temperature Can89a, Can89b, Soo87, Tra89.

To consider the exciton effect, Ganguly and Birman derived a formal expression of Raman polarizabilities Gan67. Martin further developed a Green's-function formalism to calculate the exciton effect, and also gave an explicit expression for uncorrelated e-h pairs as intermediate states for comparison [Mar71b]. Recently, A. Cantarero, Trallero-Giner, and Cardona proposed a reliable theoretical model, achieving a general expression valid in a broad spectral range around the critical point Can89a, Can89b, Tra89. All the research shows that the exciton effect plays an important role under resonant excitation condition. 


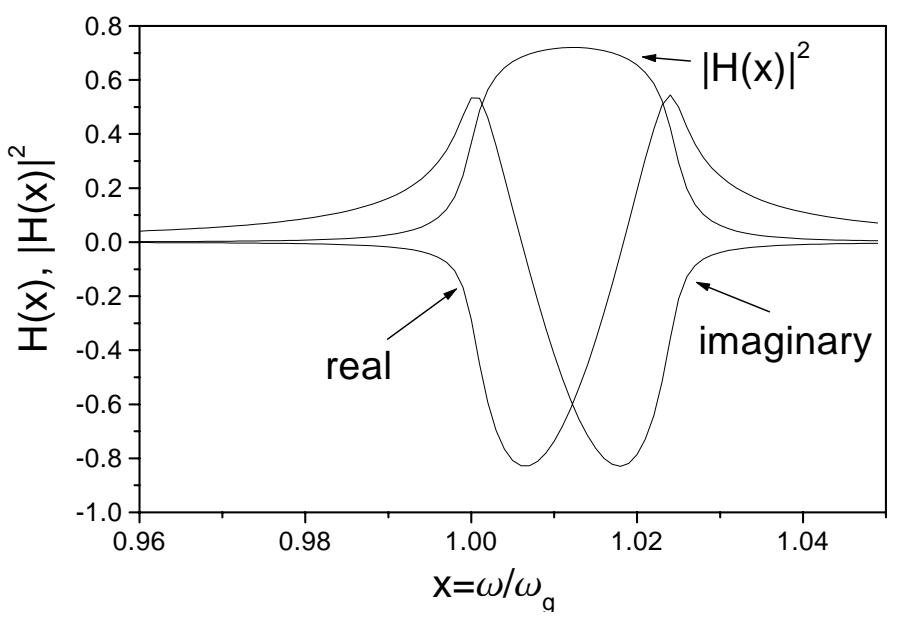

Figure 2.9: Dependence of the intraband F-induced Raman polarizability function $H(x)$ on reduced frequency.

Taking as intermediate states the interband transitions modified by excitonic electron-hole correlation suffice to reproduce more precisely the absolute values of the measured Raman polarizability of many III-V compounds.

Different from the formalism in the last section, in which Raman polarizabilities are derived from by the standard linear polarizability, here the Raman polarizabilities are obtained by using third-order perturbation theory with the aid of Feynman diagrams. The Raman polarizabilities can be expressed as Can89b]

$$
a=\frac{n_{l} n_{s}}{2 \pi} \frac{v_{0}}{\bar{u}_{0}} \frac{1}{\hbar \omega_{0}} W_{f i}\left(\omega_{s}, \hat{\mathbf{e}}_{s} ; \omega_{l}, \hat{\mathbf{e}}_{l}\right)
$$

where $\bar{u}_{0}$ is a defined relative displacement. $W_{f i}$, the amplitude probability for the resonant scattering by $1 \mathrm{LO}$ phonon, is given by

$$
W_{f i}=\sum_{\alpha, \beta} \frac{\left\langle f\left|H_{E R}\right| \beta\right\rangle\left\langle\beta\left|H_{E P}\right| \alpha\right\rangle\left\langle\alpha\left|H_{E R}\right| i\right\rangle}{\left(\hbar \omega_{l}-E_{\beta}+i \Gamma_{\beta}\right)\left(\hbar \omega_{s}-E_{\alpha}+i \Gamma_{\alpha}\right)} .
$$

The meanings of the symbols have been defined in Eq. 2.4. By inserting the wave functions of intermediate states, which are obtained by solving the Schrödinger equation including the Coulomb interaction, one thus acquires the Raman polarizabilities for the correlated e-h pairs, i.e., excitons. Therefore, one key to calculate the Raman scattering intensity is to find out wave functions of all the eigen-states, i.e., discrete and continuum excitons. The derivation from such a starting point is straightforward but lengthy. In the theory presented in Refs. [Can89a, Can89b, Tra89], the contributions are treated separately by distinguishing discrete-discrete, discrete-continuum, continuum-continuum matrix element terms. 
To introduce their theory briefly but clearly, we divide the discussion of the DP-induced RRS and F-induced RRS into two sub-sections. In each sub-section, the contributions from discrete exciton state and continuous exciton states can be easily identified in the formulation.

\section{DP-induced RRS}

With Coulomb interaction between electrons and holes, several exciton branches, in general with different Bohr radii, are generated. Here heavy, light, and split-off exciton will be taken into account using the Wannier-Mott exciton model. In the allowed Raman scattering by LO and TO phonons, these excitonic intermediate states are included in the calculation. The Raman efficiency is obtained from third-order perturbation theory.

For a three-band model and using the hydrogenic approximation for discrete and continuous exciton states the DP-induced Raman polarizability can be written in terms of contributions of critical points $E_{0}, E_{0}+\Delta_{0}$, and other nonresonant terms:

$$
\begin{aligned}
a_{D P} & =\sum_{i, j} K_{i, j}^{D P}\left\{\sum_{n=1}^{\infty} \frac{1}{n^{3}} \frac{1}{\left[\xi_{i}+1 / n^{2}+i \gamma_{i}(n)\right]\left[\xi_{i}-\xi_{0}+1 / n^{2}+i \gamma_{j}(n)\right]}\right. \\
& -\frac{1}{2} \sum_{n=1}^{\infty} \frac{1}{n^{3}} \frac{1}{\left[\xi_{i}+1 / n^{2}+i \gamma_{i}(k)\right]\left[\xi_{i}-\xi_{0}+1 / n^{2}+i \gamma_{j}(k)\right]} \\
& +\frac{1}{4} \frac{1}{\xi_{i}-\xi_{j}+\xi_{0}+i\left[\gamma_{i}(k)-\gamma_{j}(k)\right]}\left[\ln \frac{\xi_{j}-\xi_{0}+i \gamma_{j}(k)}{\xi_{i}+i \gamma_{i}(k)}\right. \\
& \left.\left.+i \pi\left[\operatorname{coth} \frac{\pi}{\left[\xi_{i}+i \gamma_{i}(k)\right]^{1 / 2}}-\operatorname{coth} \frac{\pi}{\left[\xi_{j}-\xi_{0}+i \gamma_{j}(k)\right]^{1 / 2}}\right]\right]\right\}+b_{D P}
\end{aligned}
$$

with $\xi_{i}=\left(\hbar \omega_{l}-E_{g i}\right) / E_{X} ; \xi_{0}=\hbar \omega_{0} / E_{X} ; \gamma_{i}=\Gamma_{i} / E_{X}$. Here $E_{X}$ denotes the 1-s exciton binding energy. $n$ here denots the index of discrete exciton. The index $i(j)$ refers to the exciton formed between a valence and a conduction band with gap $E_{g i}, \Gamma_{i}$ is the exciton lifetime broadening. $k$ is the charge's wave number.

The first term on the rhs of Eq. 2.31 corresponds to the discrete-discrete excitonic contribution, the second to the continuous-discrete and discrete-continuous contribution, and the last one to the continuous-continuous contribution.

The factor $K_{j, i}^{D P}$ is

$$
K_{j, i}^{D P}=\frac{a_{0}^{2}}{2 \pi \sqrt{3}} \frac{R y^{2}}{E_{X}^{2}} \frac{a_{H}^{3}}{a_{X}^{3}} \frac{\left|p_{c v}\right|^{2}}{m} \frac{C_{j i} d_{0}}{\hbar \omega_{l}\left(\hbar \omega_{l} \hbar \omega_{s}\right)^{1 / 2}},
$$

where $R y$ and $a_{H}$ are the Rydberg energy and Bohr radius of hydrogen atom, $d_{0}$ the deformation constant, $C_{j i}$ a numeral factor calculated taking the average of the oscillator strength. The sum in $i, j$ runs over heavy, light, and split-off hole bands. The assumption of the same exciton Bohr radius $a_{X}$ for all excitons formed from conduction and valence bands (hh, hl, and so) was taken. 
It has been shown that the first term of the Eq. 2.31, corresponding to the discretediscrete scattering, gives the dominant contribution for the intrinsic RRS below the $E_{0}$ band gap [Can89a].

\section{F-induced RRS}

The Raman polarizability of F-induced RRS is derived as

$$
\begin{aligned}
a_{F} & =\sum_{i} K_{i}^{F}\left\{\sum_{n, m=1}^{\infty} \frac{D_{n, m}}{\left[\xi_{i}+1 / n^{2}+i \gamma_{i}(n)\right]\left[\xi_{i}-\xi_{0}+1 / m^{2}+i \gamma_{i}(m)\right]}\right. \\
& +\sum_{n=1}^{\infty} \frac{1}{n^{3}} \int_{0}^{\infty} d k \frac{D_{n, k}}{1-e^{-2 \pi / k}}\left[\frac{1}{\left[\xi_{i}-k^{2}+i \gamma_{i}(k)\right]\left[\xi_{i}-\xi_{0}+1 / n^{2}+i \gamma_{i}(n)\right]}\right. \\
& \left.+\frac{1}{\left[\xi_{i}+1 / n^{2}+i \gamma_{i}(n)\right]\left[\xi_{i}-\xi_{0}-k^{2}+i \gamma_{i}(k)\right]}\right] \\
& +\frac{i}{8\left(q_{e}^{2}-q_{h}^{2}\right)}\left[\frac{1}{q_{e}} \ln \frac{\left[\xi_{i}+i \gamma_{i}(k)\right]^{1 / 2}+\left[\xi_{i}-\xi_{0}+i \gamma_{i}(k)\right]^{1 / 2}-q_{e}}{\left[\xi_{i}+i \gamma_{i}(k)\right]^{1 / 2}+\left[\xi_{i}-\xi_{0}+i \gamma_{i}(k)\right]^{1 / 2}+q_{e}}\right. \\
& \left.\left.-\frac{1}{q_{h}} \ln \frac{\left[\xi_{i}+i \gamma_{i}(k)\right]^{1 / 2}+\left[\xi_{i}-\xi_{0}+i \gamma_{i}(k)\right]^{1 / 2}-q_{h}}{\left[\xi_{i}+i \gamma_{i}(k)\right]^{1 / 2}+\left[\xi-\xi_{0}+i \gamma_{i}(k)\right]^{1 / 2}+q_{h}}\right]\right\},
\end{aligned}
$$

where $q_{\alpha}=\left(m_{\alpha} / m_{e-h}\right) q a_{X}(\alpha=e, h), m_{e-h}=m_{e}+m_{h}$, and the definition of the other symbols are the same as in Eq. 2.33. The terms $D_{n, m}$ and $D_{n, k}$ are proportional to the matrix elements corresponding to discrete-discrete and discrete-continuous exciton transitions, respectively. The coefficient $K_{i}^{F}$ in Eq. 2.33 is given by

$$
K_{i}^{F}=-\frac{2}{\pi}\left[a_{0}^{3} \frac{M}{m} \frac{\hbar \omega_{0}}{R y}\right]^{1 / 2} \frac{q a_{H}}{\hbar \omega_{l}\left(\hbar \omega_{l} \hbar \omega_{s}\right)^{1 / 2}} \frac{2}{3} \frac{\left|p_{c v}\right|^{2}}{m} \frac{a_{H}}{a_{X}}\left[\frac{R y}{E_{X}}\right]^{2} i C_{F} \frac{m_{e}-m_{h}}{m_{e-h}}
$$

where $C_{F}$ is given by Eq. 2.11.

The last term inside the curly brackets in Eq. 2.33 represents the contribution due to the continuous states that has been evaluated using the free e-h wave functions.

Similar to the case of DP-induced scattering, the first term of the Eq. 2.33 corresponds to the discrete-discrete scattering, which has been shown to be the dominant contribution for the intrinsic RRS below the $E_{0}$ band gap [Tra89].

\subsection{Measurement of Raman scattering cross-sections}

In Raman scattering experiments, the measurable value is the scattering efficiency or rate $S$, which is defined as the power ratio of scattered light $P_{s}$ to incident light $P_{l}$ per length $L$ in solid angle $\Omega$ (Fig. 2.10):

$$
S=\frac{P_{s}}{L \cdot P_{l}} \Omega
$$




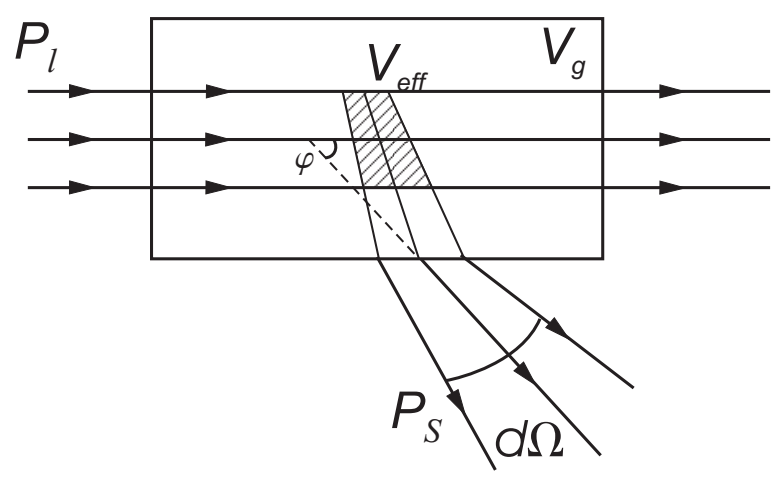

Figure 2.10: Measurement of Raman scattering efficiency. $V_{\text {eff }}$ is the effective scattering volume. The differential scattering rate per unit cubic angle is measured outside the crystal.

Since Raman scattering efficiency relates to Raman tensor directly, by measuring Raman scattering efficiency one can obtain the Raman polarizability. Notice that in Raman experiments using photon counting electronics, one actually measures the scattered photon rate outside the crystal, so that the incident (scattered) light transmission coefficients $\left(T_{l(s)}\right)$ and absorption coefficients $\left(\alpha_{l(s)}\right)$ should be taken into account. The measured scattering rate $S^{\prime}$ in solid angle $\Delta \Omega^{\prime}$ outside the crystal relates to the Raman tensor by [Men85, Soo87]

$$
S^{\prime}=\frac{T_{s} T_{l} P_{l} \omega_{s}^{3}\left[1-e^{-\left(\alpha_{l}+\alpha_{s}\right) L}\right](1+n) \Delta \Omega^{\prime}}{2 c^{4}\left(\alpha_{s}+\alpha_{l}\right) \eta_{s} \eta_{l} M \omega_{0} v_{0}}\left|\hat{\mathbf{e}_{s}} \cdot \mathbf{R}(q) \cdot \hat{\mathbf{e}_{l}}\right|^{2}
$$

where $M$ is the reduced mass of $\mathrm{PC}, n$ the phonon occupation number; $\eta_{l(s)}, \omega_{l(s)}$ the refractive index, frequency of the laser (scattered) light, respectively.

According to the expression of Eq. 2.36, the absolute scattering cross section can be calculated if all the components in the system are known. However, most experimental measurements of absolute cross sections in semiconductors employ the sample substitution method, in which the calibration is carried out by comparing the sample with a standard known scattering efficiency. The advantage of substitution method lies in the fact that the geometry measurement errors are cancelled. We applied the sample substitution method in our experiments. A pure bulk Si sample was used as a reference. We also referred to the known intrinsic RRS data of high-purity GaAs for our measurement calibration.

A rough estimate of the order of scattering cross-section (non-resonance) can be made: it is $10^{-30} \mathrm{~m}^{2}$ for a single atom and about $10^{-4}-10^{-5} \mathrm{~m}^{2}$ for a cubic meter of crystal Hay78. 


\section{Chapter 3}

\section{Experimental}

\section{$3.1 \quad$ Set-up}

The experimental set-up is sketched in Fig. 3.1.

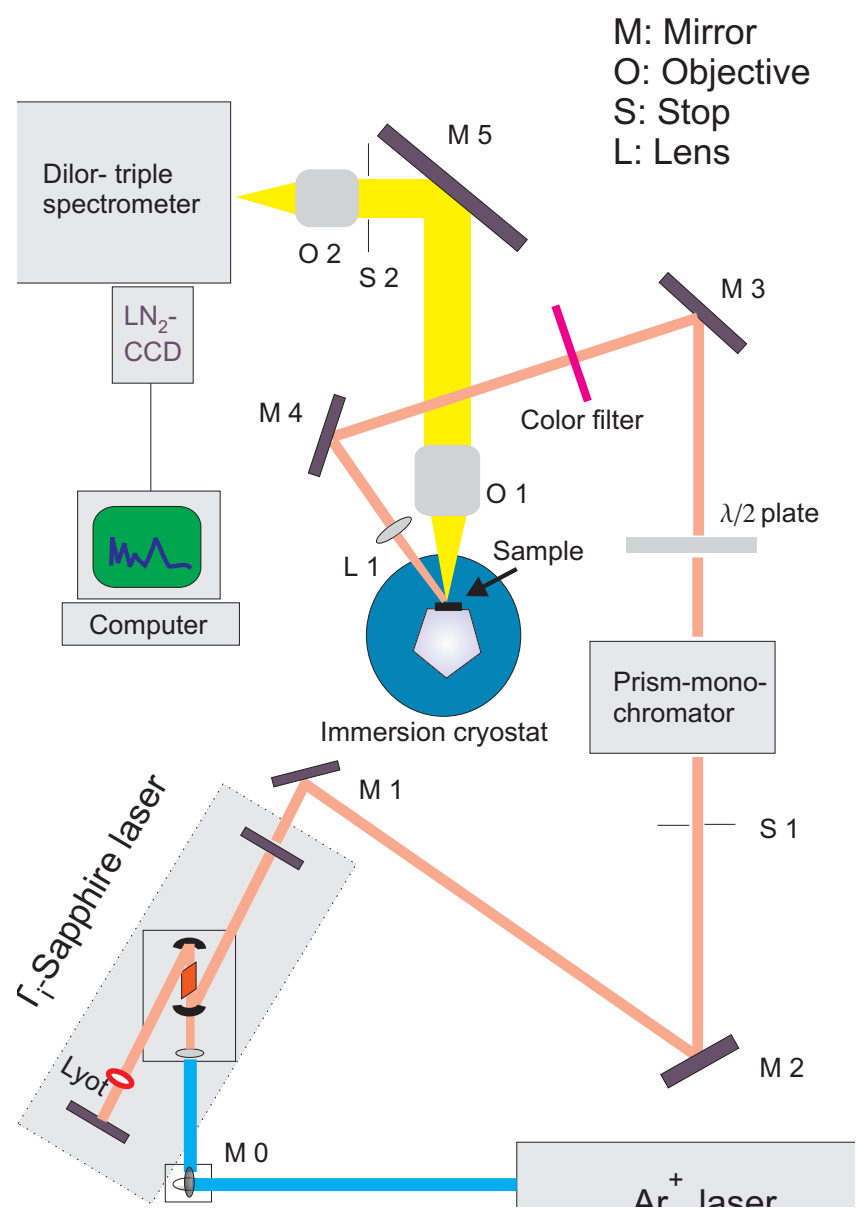

Figure 3.1: Experimental set-up. 
The main components are explained in the following:

Laser: a self-made $\mathrm{Ti}^{3+}$-Sapphire laser 11 was used as a tunable excitation source. A commercial two-quartz-plate Lyot-filter was inserted in the cavity for monochromatic mode tuning. In the wavelength range 720-890 nm, one of the cavity end-mirrors is high reflective while the other, i.e., the output coupling mirror has $2 \%$ transmission efficiency. The stable tuning wavelength range is 780-870 $\mathrm{nm}(1.588-1.424 \mathrm{eV})$. The pumping source is $\mathrm{Ar}^{+}$laser, operating in all-lines mode. Typically, $10-100 \mathrm{~mW}$ power of $\mathrm{Ti}^{3+}$-Sapphire laser was used in the experiment.

Spectrometer: in Raman scattering, the elastically scattered light is normally 4-6 orders of magnitude stronger than the Raman signal meanwhile the frequency separation between the laser light and scattered light is fairly small compared to the laser frequency (about $1 \%$ of the laser frequency). Thus Raman scattering experiments require a spectrometer with an excellent stray light rejection capability. A $0.6 \mathrm{~m}$ triple grating spectrometer (Dilor) was employed in the experiments. The stray light rejection ratio is $10^{-4}-10^{-6}$. The subtract-mode of the spectrometer was utilized for optimal flux of the scattering light. For the resolution in such a mode, the full width of half maximum (FWHM) of a laser line in spectrum is about $2 \mathrm{~cm}^{-1}$ with the slit being $150 \mu \mathrm{m}$ wide.

Cryostat: a self-made immersion cryostat was installed to carry out measurements at low temperature. When the chamber is filled with liquid helium and pumped to a low pressure level, the superfluid helium state is formed, thus the temperature can be kept at about $2 \mathrm{~K}$

Detector: to record the spectra in a multi-channel manner, a commercial liquidnitrogen-cooled charge-coupled-device (CCD) (Wright Company) was utilized as the photon detector. The noise level of the CCD detector is less than 2 counts/second per pixel.

$\lambda / 2$ plates: in order to measure RRS cross-sections in different configurations, a $\lambda / 2$ plates were inserted in the optical arrangement to adjust polarization directions of the light.

For the consideration of light polarizations, notice that, to pump the $\mathrm{Ti}^{3+}$-Sapphire laser, a pumping light with polarization direction parallel to the plane of paper is demanded in this experiment. However, the out-put light of $\mathrm{Ar}^{+}$laser has a polarization direction perpendicular to the plane of the paper. So a mirror set "M0" (see Fig. 3.1) was used to switch the polarization of the pumping light to the desired direction. Also notice that, the polarization direction of the scattered light detected by the spectrometer, however, is fixed, parallel to the slit-parallel direction, due to that fact that the gratings inside the spectrometer are much more sensitive to the light polarized in this direction than the light polarized in the slit-vertical direction.

Sample: commercial GaAs (Wacker-Chemitronic GmbH, named as 21412) was used in the experiments. The GaAs wafer has a (001) surface and the layer is $450 \pm 50 \mu \mathrm{m}$ thick. It is partially compensated GaAs with shallow donors and acceptors. It is p-type GaAs and the acceptors are mostly carbon. The impurity concentration: $N_{D}+N_{A} \sim 10^{16} \mathrm{~cm}^{-3}$. The density of filled acceptors at $2 \mathrm{~K}$ is about $6 \times 10^{15} \mathrm{~cm}^{-3}$, estimated based on the comparison

\footnotetext{
${ }^{1}$ It was designed by Dr. M. J. Gregor and P. G. Blome. Many thanks for the help in using this laser.
} 
of our absorption measurement with the calculation according to Dumke's formulation of $\left(e, A^{0}\right)$ absorption coefficient [Dum63]. This will be explained in detail in Chapter 4.

\subsection{Procedure}

The exciting laser was tuned from $1.46 \mathrm{eV}$ to $1.52 \mathrm{eV}$ for our purpose of RRS investigation. An excitation power density larger than $100 \mathrm{~W} / \mathrm{cm}^{2}$ was applied (the exciting spot size is about $100 \mu \mathrm{m}$ in radius). The sample was kept at $2 \mathrm{~K}$, immersed in the liquid helium cryostat. Back scattering geometry was taken and the incident laser light was focused perpendicularly on the sample's (001) surface. Due to large refractive index of GaAs, the angle error for non-vertical geometry inside the crystal is less than $5^{\circ}$. The scattered light was collected into the Dilor triple-grating spectrometer and detected by the CCD.

Different back-scattering configurations were adopted for different purposes. Conventionally (i.e., for intrinsic RRS), one can measure the (DP+EO)-induced LO-phonon scattering in the $Z(X Y) \bar{Z}$ configuration and the F-induced forbidden LO scattering in the $Z(X X) \bar{Z}$ configuration. TO scattering is forbidden in the back-scattering geometry. However, for impurity-induced RRS, selection rules could be revised or broken.

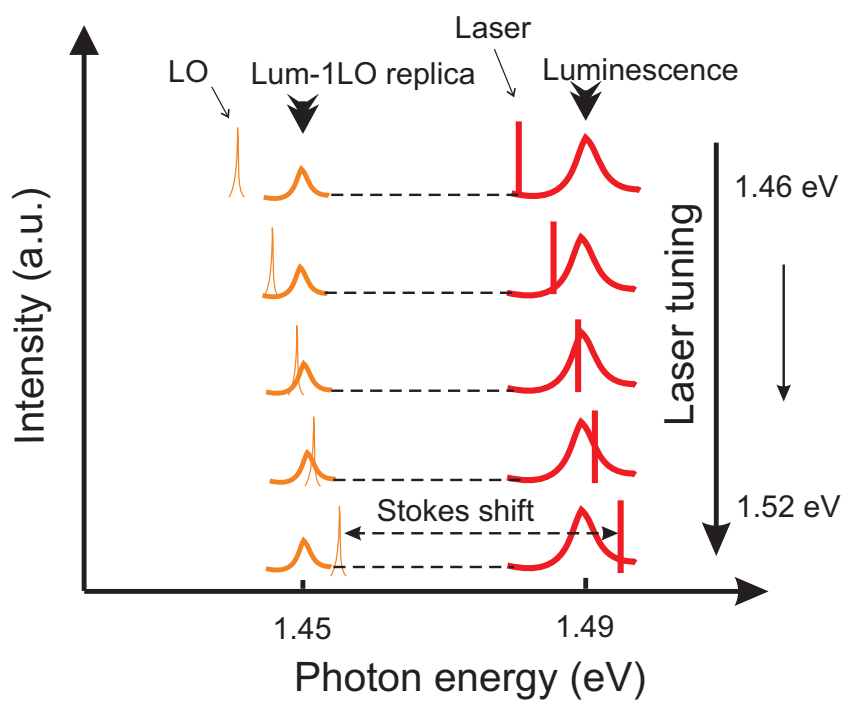

Figure 3.2: RRS spectrum recording: the broader peaks around $1.49 \mathrm{eV}$ and $1.45 \mathrm{eV}$ are ascribed to impurity luminescence and its LO phonon replicas, respectively; the sharp line with a definite Stokes shift to the laser line is due to Raman scattering. So in the recorded RRS spectra, both LO Raman scattering line and LO phonon replicas of luminescence appear and may overlap seriously with each other under resonant excitation condition. The RRS spectra are plotted in absolute energy value in unit $\mathrm{eV}$.

In order to display the results in absolute values of Raman scattering intensity, we applied sample-substitution method. We used a high-purity c-Si for calibration. The 
Raman polarizability of c-Si is about $27 \AA$ at $1.50 \mathrm{eV}$ at $77 \mathrm{~K}$ Gri80, Wag83, with the absorption coefficient about $2.0 \times 10^{2} \mathrm{~cm}^{-1}$ [Das55]. We first did RRS experiments on both the c-Si sample and the GaAs sample at $77 \mathrm{~K}$, then we calibrated the GaAs RRS data by correcting the measured scattering efficiency for absorption, refractive index, and reflectivity according to Eq. 2.36. We found that the calibrated data, which comes mainly from intrinsic scattering, was in agreement with the well-established Raman scattering parameters for high-purity GaAs measured at $77 \mathrm{~K}$. We then did experiments at $2 \mathrm{~K}$, and calibrated the RRS data at $2 \mathrm{~K}$ according to Eq. 2.36 with reference of our GaAs RRS data at $77 \mathrm{~K}$. The absorption coefficient of the GaAs sample at $2 \mathrm{~K}$ near $\left(e, A^{0}\right)$ was obtained from our own absorption measurement. The error in the correction is estimated to be about $50 \%$ due to the uncertainty of the calibrating data of c-Si and GaAs.

To make the results presented in the next chapters more understandable, Fig. 3.2 demonstrates schematically how the experiments were performed and which energy region of the spectra is of the interest. Because strong impurity luminescence is also recorded in the investigation of the impurity-induced RRS, care should be taken in the data treatment. From Fig. 3.2 we see that, for the luminescence and its phonon replicas, the energy positions are fixed, independent of exciting energy; but for the Raman scattering, the scattered light has a fixed energy difference with respect to the laser energy. So when the laser is tuned, the Raman line is shifted, too. Also notice that the LO scattering peak overlaps strongly the phonon replica signal when the laser is tuned around $1.49 \mathrm{eV}$, where $\left(e, A^{0}\right)$ or $\left(D^{0}, A^{0}\right)$ is excited. 


\section{Chapter 4}

\section{Luminescence and absorption of the GaAs sample}

\subsection{Photoluminescence}

Photoluminescence (PL) spectra are useful to investigate the optical transitions in semiconductors. The PL spectrum in Fig. 4.1 was obtained by exciting the sample 21412 with $\mathrm{Ar}^{+}$laser $(514 \mathrm{~nm})$ at $2 \mathrm{~K}$. The excitation excitation energy $E_{l}(2.41 \mathrm{eV})$ is much higher than the gap energy $E_{0}$. It is was high-intensity excitation measurement because an exciting intensity bigger than $100 \mathrm{~mW} / \mathrm{cm}^{2}$ was applied in the experiment.

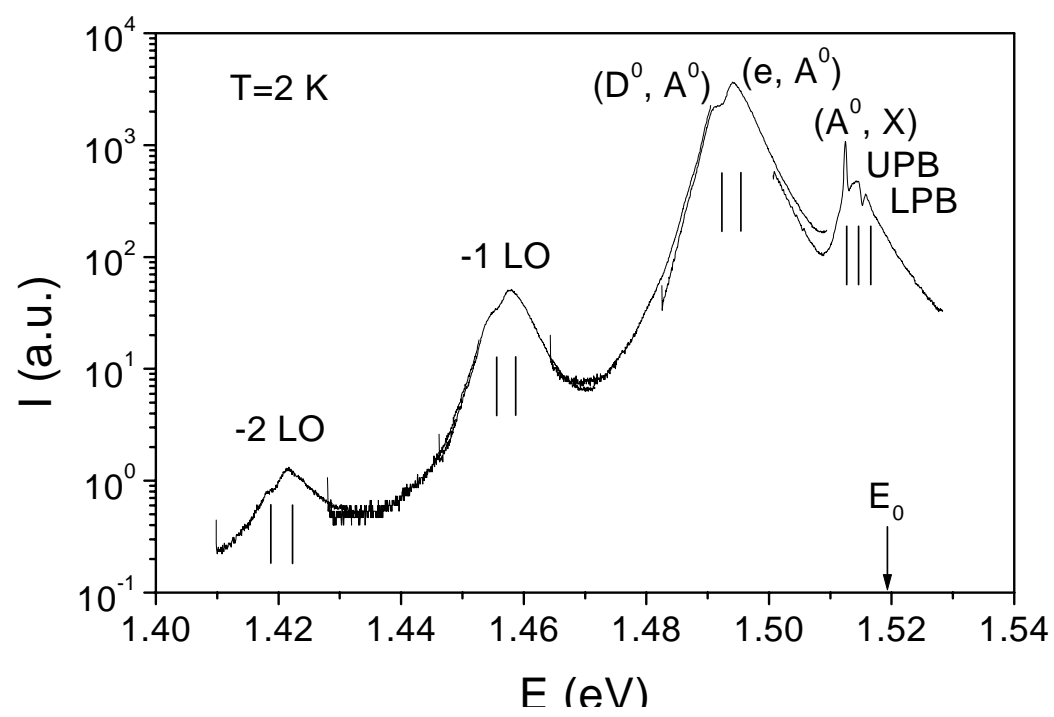

Figure 4.1: PL spectrum of GaAs below the $E_{0}$ gap at $2 \mathrm{~K}$. The impurity hot luminescence and its 1-LO and 2-LO phonon replicas have been observed, with the intensity ratios about 3500:50:1.2. 
From the PL spectrum the impurity-related transitions $\left(e, A^{0}\right),\left(D^{0}, A^{0}\right),\left(A^{0}, X\right)$, and the luminescence phonon replicas have been clearly identified.

\subsubsection{Free-to-bound transition $\left(e, A^{0}\right)$}

Electrons in the conduction band can recombine with holes bound to acceptors, and holes in the valence band can recombine with electrons bound to donors. These recombinations are called free-to-bound transitions. In the case of our p-type GaAs sample, the excited free electrons recombine with the holes at the neutral acceptor level. The peak at 1.4944 $\mathrm{eV}$ is ascribed to $\left(e, A^{0}\right)$ transition. The acceptors are mostly carbon with binding energy $E_{A}=27 \mathrm{meV}$. According to the parameters in Appendix A, the band gap is $1.52 \mathrm{eV}$, so the emission line would appear at $1.493 \mathrm{eV}$ if the emission starts from the conduction band minimum and ends at the acceptor level. However the $\left(e, A^{0}\right)$ peak position is at $1.4944 \mathrm{eV}$ in the PL spectrum, a little higher than $1.493 \mathrm{eV}$, see Fig. 4.1. This is because we employed high exciting intensity in the experiment. Because the electrons are excited with kinetic energies much higher than the conduction band minimum, the temperature of these electrons is thus higher than the temperature of the lattice. They are called hot electrons. These electrons form non-degenerate electon gas and distribute above the bottom of the conduction band after energy relaxation. Therefore the photons are emitted with energy higher than the energy of emission which starts from the band-minimum in the recombination. It was also observed in our experiments that the luminescence was shifted to lower energy with decrease of excitation intensity. With a smallest excitation intensity one can observe that the luminescence peak is approaching $1.493 \mathrm{eV}$ [Ulb78].

The $\left(e, A^{0}\right)$ recombination is described by

$$
e+A^{0} \longrightarrow A^{-}+\hbar \omega
$$

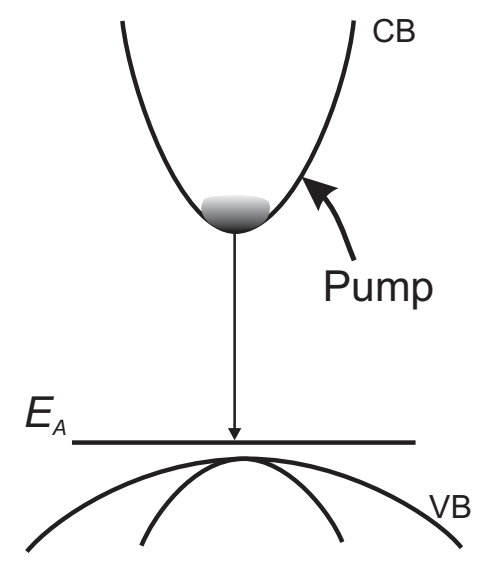

Figure 4.2: Schematic illustration of $\left(e, A^{0}\right)$ recombination. $E_{A}$ is the acceptor level with the energy origin assigned at the top position of the valence band. 


\subsubsection{Bound exciton $\left(A^{0}, X\right)$}

Bound excitons are formed when excitons, i.e., electron-hole pairs, are trapped at impurities. At low temperature, recombination via the bound exciton is a principal decay route for electrons and holes at non-equilibrium states. In p-type GaAs, excitons are bound to neutral acceptors at low temperature. The recombination of the bound excitons gives out emission light with energy less than the emission energy of free excitions because additional binding energy is included in the bound excitons. This recombination can be described by

$$
\left(A^{0}, X\right) \longrightarrow A^{0}+\hbar \omega
$$

The peak position $E_{\text {lum }}$ of the emission $\left(A^{0}, X\right)$ can be computed by

$$
E_{l u m}=E_{0}-E_{X}-E_{B X}
$$

where $E_{B X}$ is the additional binding energy for the exciton bound to the neutral acceptor. $E_{X}$ is the binding energy of a 1-s free exciton, which is taken as $4.2 \mathrm{meV}$ at $2 \mathrm{~K}$ (see Appendix A). As shown by the strong and sharp peak at $1.5125 \mathrm{eV}$ in the PL spectrum in Fig. 4.1, the acceptor bound exciton $\left(A^{0}, X\right)$ is dominant in the sample 21412. From Eq. 4.1 we find that the binding energy of $\left(A^{0}, X\right)$ is about $2.5 \mathrm{meV}$.

Besides the peak at $1.5125 \mathrm{eV}$, one can also see two small peaks around $1.515 \mathrm{eV}$ with a "dip" between them. They are related to the recombination of 1-s excitons. The "dip" or "break" of the peaks is due to the exciton-polariton effect. When the coupling between excitons and photons is strong enough, the coupled system cannot be regarded as independent elementary excitations any more. At the point where the photon dispersion curve intersects the exciton dispersion curves, the energy degeneracy of the exciton and the photon is lifted by the exciton-photon coupling and the dispersion curve is splitted into two branches, see Fig. 4.3. Reflected in the emission spectrum, the peak at higher energy position corresponds to the bottom of the upper polariton branch (UPB), whereas the peak at lower energy position corresponds to the "bottle-neck" of the lower polariton branch (LPB).

\subsubsection{Bound-to-bound recombination $\left(D^{0}, A^{0}\right)$}

In the case where donor and acceptor impurities have comparable concentrations in the crystal, compensation happens and gives rise to ionized impurities at low temperature. Also, the compensation happens after the exciting light re-fills the impurity levels. This compensation process can be radiative, in which electrons bound to donors recombine with holes bound to acceptors, emitting light with energies less than that of $\left(e, A^{0}\right)$. So this is bound-to-bound recombination. The recombination is denoted by $\left(D^{0}, A^{0}\right)$ with the meaning:

$$
D^{0}+A^{0} \longrightarrow D^{+}+A^{-}+\hbar \omega
$$




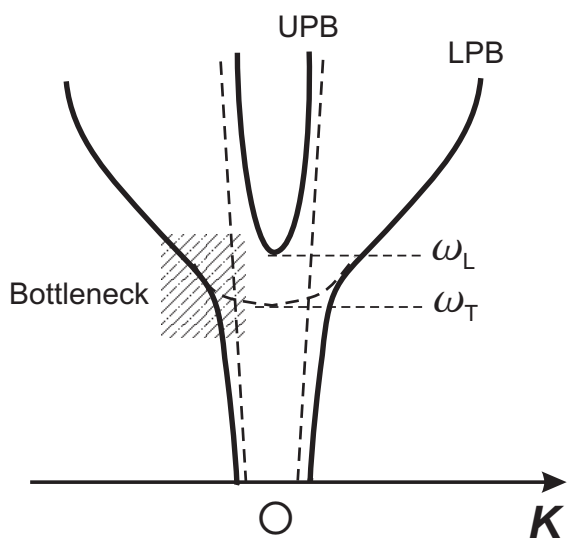

Figure 4.3: Exciton-polariton effect in two-particle picture. The solid curve represents the dispersion of an polariton. $\boldsymbol{K}$ is the wave vector of exciton. UPB and LPB stand for the upper polariton branch and lower polariton branch, respectively. The emission positions at $\omega_{L}$ and $\omega_{T}$ from upper and lower branches of the polariton have been notified in the plot.

In the PL spectrum Fig. 4.1, one sees that a lower energy "shoulder" appears at $1.4915 \mathrm{eV}$, near the strong and broad peak at $1.4944 \mathrm{eV}$. This "shoulder" signal is just ascribed to the $\left(D^{0}, A^{0}\right)$ recombination. The donor level is at about $5.8 \mathrm{meV}$ below the conduction band minimum (Appendix A). For high-purity GaAs $\left(N_{D}+N_{A} \sim 10^{12} \mathrm{~cm}^{-3}\right)$, with sufficiently low intensity excitation, $\left(D^{0}, A^{0}\right)$ would appear as a peak at about 1.489 $\mathrm{eV}$ [Ulb73, Ulb78, which corresponds to the donor acceptor pairs (DAP) with a large mean distance $\left(\sim 10^{4} \AA\right)$ where the Coulomb potential between the pairs is negligible. However, for the lightly-doped GaAs sample (our sample has the mean separation about $600 \AA$ ) and under high-intensity excitation condition, the luminescence of $\left(D^{0}, A^{0}\right)$ is shifted to higher side. This blue-shift can be easily understood by considering that closer pairs (in which the Coulomb potential should be considered) are excited, and these closer pairs have a larger radiative recombination rate. Chapter 5 will explain the dependence of $\left(D^{0}, A^{0}\right)$ luminescence energy on DAP separation in the real space in more detail.

\subsubsection{Phonon replicas}

The line at $1.458 \mathrm{eV}$ is ascribed to $\left(e, A^{0}\right)-1 \mathrm{LO}$ phonon replica. It has an energy shift $36.5 \mathrm{meV}$ with respect to the $\left(e, A^{0}\right)$ line, which is exactly the LO phonon energy at $2 \mathrm{~K}$. Also, the peak has a lower energy shoulder, which is attributed to $\left(D^{0}, A^{0}\right)-1$ LO phonon replica. The phonon replicas will be discussed in detail in Chapter 7 .

\subsection{Absorption}

The optical density (O.D.) can be measured according to 


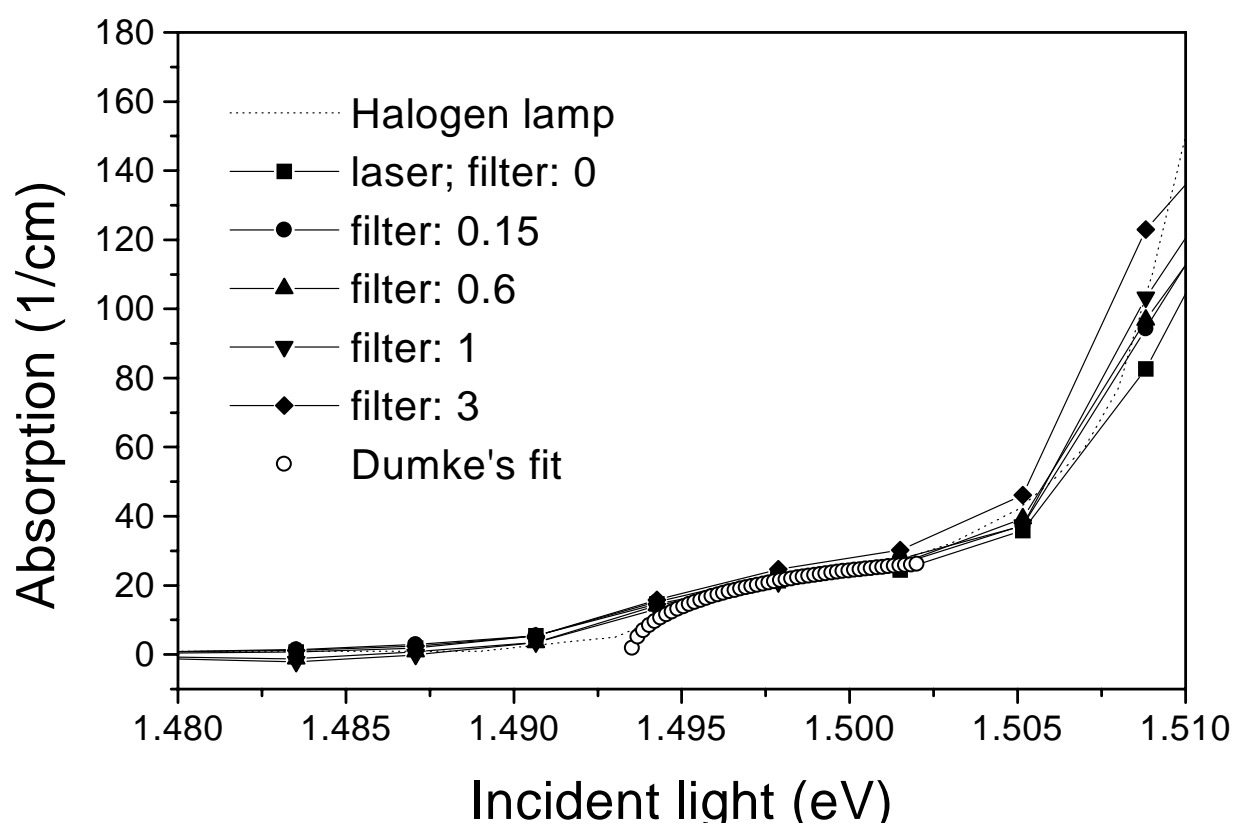

Figure 4.4: Absorption measurements of the p-type GaAs sample 21412 at $2 \mathrm{~K}$ with different excitation intensity. The absorption in the region of $1.494-1.502 \mathrm{eV}$ is mainly due to $\left(e, A^{0}\right)$. The absorption enhancement above 1.505 is mainly due to $\left(A^{0}, X\right)$. No pronounced difference for the absorption of $\left(e, A^{0}\right)$ can be identified at different excitation intensities. The $\left(e, A^{0}\right)$ absorption can be explained well by applying Dumke's theory, see the calculated circle dots, where the acceptor concentration about $6 \times 10^{15} \mathrm{~cm}^{-3}$ is thus deduced.

$$
\text { O.D. }=-\log \frac{I_{t}}{I_{0}},
$$

where $I_{0}$ and $I_{t}$ are intensities of incident light and intensity of transmission light, respectively.

The optical density relates to the absorption coefficient $\alpha$ by

$$
\text { O.D. }=-\log \left[\frac{(1-\mathcal{R})^{2}}{1-\mathcal{R}^{2}} \exp (-\alpha d)\right],
$$

where $d$ is the specimen's thickness, and $\mathcal{R}$ is the reflection coefficient, which is a function of refractive index $\eta$ :

$$
\mathcal{R}=\left[\frac{\eta-1}{\eta+1}\right]^{2}
$$

The measured absorption coefficient for the $(450 \pm 50) \mu m$-thick sample is shown in Fig. 4.4. Both a Halogen lamp (white light) and tunable Ti-Sapphire laser (monochromic light) 
were used in the measurement of transmission ratio. Accordingly, the exciting intensity was changed from $1 \mathrm{~mW} / \mathrm{cm}^{2}$ to $10^{3} \mathrm{~mW} / \mathrm{cm}^{2}$ by using a series of gray filters. It is found that the $\left(e, A^{0}\right)$ absorption within the energy region $1.494-1.505 \mathrm{eV}$ does not depend essentially on the excitation intensity. The absorption beyond $1.505 \mathrm{eV}$ results mainly from $\left(A^{0}, X\right)$, where the data show somewhat difference in absorption coefficient when the excitation intensity is changed. However, the data in this energy region are not reliable because the transmission from the thick sample was too weak to be detected by the solar cell precisely in the experiment. For the measurement of absorption due to $\left(A^{0}, X\right)$, only thinner samples could provide reliable results.

The ionized acceptor concentration can be estimated by applying the Dumke's theory to the $\left(e, A^{0}\right)$ absorption measurement. The quantitative description of $\left(e, A^{0}\right)$ transition will be given in Chapter 5. Here we mention that the acceptor concentration $N_{A^{-}}$is estimated to be $6 \times 10^{15} \mathrm{~cm}^{-3}$ at $2 \mathrm{~K}$ for the sample 21412 according to Dumke's formulation. 


\section{Chapter 5}

\section{Impurity-induced radiative transitions}

This thesis deals with impurity-induced Raman scattering. Since the scattering involves generation and recombination of e-h pairs, we survey the impurity-related transitions quantitatively in this chapter.

\subsection{Intrinsic transitions}

Before analyzing impurity-related, i.e., extrinsic radiative transitions, we first analyze intrinsic radiative transitions for two reasons:

- the description for the extrinsic radiative interband transitions can be deduced in a similar manner as for intrinsic radiative transitions;

- the comparison between the impurity transitions and the intrinsic transitions facilitates us to understand the large scattering intensity of the impurity-induced RRS.

In order to describe the transition rate quantitatively, the term of oscillator strength can be applied conveniently. From oscillator strength we can also obtain other optical parameters like absorption coefficient. In the following we will discuss the oscillator strength and absorption coefficient jointly because of their close relationship.

In general, the oscillator strength is defined as Osb79.

$$
f=\frac{2}{\hbar \omega m}\left|\left\langle i\left|P_{Z}\right| f\right\rangle\right|^{2}
$$

where $P_{Z}$ is the dipole operator between the initial state $\mid i>$ and the final state $\mid f>$. The oscillator strength can be regarded as the "number" of oscillators with the oscillation frequency $\omega$. From Eq. 5.1 the oscillator strength can be calculated if the wave functions of the initial state and final state are known. 


\subsubsection{Uncorrelated e-h pairs}

Let us first treat the optical transition from free e-h pairs in a semiconductor. The wave function of electron (hole) in an uncorrelated e-h pair (without Coulomb interaction) is given by

$$
\Psi_{e(h)}(\boldsymbol{r})=\frac{e^{i \boldsymbol{k} \cdot \boldsymbol{r}}}{\sqrt{V}_{g}} u_{c(v) 0}(\boldsymbol{r})
$$

where $V_{g}$ is the volume of the whole crystal, $u_{c(v) 0}(\boldsymbol{r})$ is the periodic part of the Bloch wave function of conduction (valence) band, $\boldsymbol{r}$ and $\boldsymbol{k}$ the coordinator and wave vector of the electron (hole), respectively.

The oscillator strength of the optical transition between conduction band electrons and valence band holes is obtained by calculating Eq. 5.1 with the wave functions given in Eq. 5.2 :

$$
f_{c v}=\frac{2\left|p_{c v}\right|^{2}}{m E_{0}}
$$

where Pey93

$$
p_{c v} \equiv<u_{c 0}\left|\hat{\mathbf{e}}_{\mathbf{q}} \cdot \boldsymbol{p}\right| u_{v 0}>=i m \omega<u_{c 0}\left|\hat{\mathbf{e}}_{\mathbf{q}} \cdot \boldsymbol{r}\right| u_{v 0}>\quad .
$$

$\left|p_{c v}\right|^{2}$ can be estimated approximately by $\hbar\left(2 \pi / a_{0}\right)$ [Yu99. For GaAs, according to the relevant parameters listed in Appendix A, $\left|p_{c v}\right|^{2}=21(\mathrm{eV})$. Thus the oscillator strength $f_{c v}$ is about 14.5 .

The absorption coefficient for uncorrelated e-h pairs of GaAs at the band gap is derived as Pey93

$$
\alpha_{e-h}(E=\hbar \omega)=\frac{2 e^{2}}{\eta c m^{2} \omega}\left|p_{c v}\right|^{2}\left(\frac{2 \mu}{\hbar^{2}}\right)^{3 / 2}\left(E-E_{0}\right)^{1 / 2} \theta\left(E-E_{0}\right)
$$

where $\theta(E)$ is a step function, defined as

$$
\theta\left(E-E_{0}\right)=\left\{\begin{array}{ll}
1 & E-E_{0} \geq 0 \\
0 & E-E_{0}<0
\end{array} .\right.
$$

As seen from Eq. 5.5, the absorption coefficient is proportional to $\left|p_{c v}\right|^{2}$, accordingly, the oscillator strength $f_{c v}$. Normally, the absorption coefficient can be measured directly in the absorption experiment. However, the absorption of uncorrelated e-h pairs of GaAs can not be measured directly due to the dominant absorption of continuum excitons above the $E_{0}$ gap.

\subsubsection{Free exciton}

Now we consider the case of free excitons. The wave function of a free 1-s exciton is given by 


$$
\Psi_{X}(\boldsymbol{r}, \boldsymbol{R})=\frac{1}{\sqrt{V_{g}}} e^{i \boldsymbol{K} \cdot \boldsymbol{R}}\left(\frac{1}{\sqrt{\pi a_{X}^{3}}}\right) e^{-r / a_{X}} u_{c 0}\left(\boldsymbol{r}_{e}\right) u_{v 0}\left(\boldsymbol{r}_{h}\right)
$$

where $a_{X}$ is the Bohr radius of the free exciton, $\boldsymbol{K}=\boldsymbol{k}_{e}+\boldsymbol{k}_{h}, \boldsymbol{r}=\boldsymbol{R}_{e}-\boldsymbol{R}_{h}, \boldsymbol{R}=$ $\left(m_{e} \boldsymbol{R}_{e}+m_{h} \boldsymbol{R}_{h}\right) /\left(m_{e}+m_{h}\right)$ ( $\boldsymbol{R}$ is thus called the exciton's center-of-mass coordinate).

In analogy with a hydrogen atom, for the 1-s exciton we have

$$
E_{X}=\frac{\hbar^{2}}{2 \mu a_{X}^{2}}
$$

and

$$
a_{X}=\frac{\hbar^{2} \epsilon_{0}}{e^{2} \mu}=\frac{e^{2}}{2 \epsilon_{0} E_{X}}
$$

where $E_{X}$ is the exciton bing energy. If taking $E_{X}=4.2(\mathrm{meV})$ (Appendix A), we have $a_{X} \simeq 137 \AA$ according to Eq. 5.9.

Using the wave function in Eq. 5.7 and referring to the book [Yu99] for the method to calculate the matrix element in Eq. 5.1, so we obtain the 1-s exciton's oscillator strength in the form:

$$
f_{X}=\frac{v_{0}}{v_{X}} f_{c v}
$$

where $v_{X}=\pi a_{X}^{3}$, standing for the exciton relative motion volume. For GaAs, using $a_{X} \simeq 137 \AA$ we get $f_{X} \simeq 8 \times 10^{-5}$.

The absorption coefficient $\alpha_{X}(E)$ including the exciton effect is given by [Ell57, Pey93

$\alpha_{X}(E=\hbar \omega)=\alpha_{0} \frac{E}{E_{X}}\left[\sum_{n} \frac{4 \pi}{n^{3}} E_{X} \delta\left(E-E_{0}+E_{X}\right)+\theta\left(E-E_{0}\right) \frac{\pi e^{\pi / \sqrt{\left(E-E_{0}\right) / E_{X}}}}{\sinh \left(\pi / \sqrt{\left(E-E_{0}\right) / E_{X}}\right)}\right]$,

where

$$
\alpha_{0}=\frac{2 e^{2} p_{c v}^{2}}{m^{2} \eta c \hbar \omega^{2} a_{X}^{3}}=\frac{e^{2}}{\eta c m \omega a_{X}^{3}} f_{c v}
$$

and $\theta\left(E-E_{0}\right)$ has been explained by Eq. 5.6.

With $\alpha_{0}$ defined in Eq. 5.12, the absoption coefficient for free e-h pairs given by Eq. 5.5 can also be re-written as

$$
\alpha_{e-h}(E)=\alpha_{0} \frac{E}{E_{X}^{3 / 2}}\left(E-E_{0}\right)^{1 / 2} \theta\left(E-E_{0}\right) \propto f_{c v}\left(E-E_{0}\right)^{1 / 2} \theta\left(E-E_{0}\right)
$$

According to the known parameter values (see Appendix A), we have $\alpha_{0} \simeq 5 \mathrm{~cm}^{-1}$.

The absorption due to continuum excitons at $E_{0}$ is given by the second term on the rhs of Eq. 5.11: 


$$
\alpha_{C}=2 \pi \alpha_{0} \frac{E}{E_{X}}
$$

The calculation of Eq. 5.14 predicts $\alpha_{C} \approx 1.1 \times 10^{4} \mathrm{~cm}^{-1}$. From experiments, $\alpha_{C} \approx$ $8 \times 10^{3} \mathrm{~cm}^{-1}$ [Tri88, so it is consistent with the predicted value.

Moreover, we can also reckon the oscillator strength of the discrete 1-s exciton from the absorption coefficient obtained from the absorption line if we assume a Lorentzian line shape for it, i.e.,

$$
\mathcal{L}(E)=A \cdot \frac{\Gamma / 2 \pi}{\left(E-E_{0}+E_{X}\right)^{2}+(\Gamma / 2)^{2}}
$$

where $A$ is the area and $\Gamma$ the FWHM of the peak. $A$ is given by

$$
A=2 \alpha_{C} E_{X}
$$

So the absorption coefficient maximum of the 1-s exciton is evaluated as

$$
\alpha_{X}^{\max }\left(E=E_{0}-E_{X}\right)=8 \alpha_{0} \frac{E_{0}-E_{X}}{\Gamma} .
$$

Taking $\Gamma \sim 3 \mathrm{meV}$ in Eq. 5.17, we have absorption coefficient $\alpha_{X}^{\max } \sim 2 \times 10^{4} \mathrm{~cm}^{-1}$, which is in agreement with the experimental value $\alpha_{X}^{\max }=1.4 \times 10^{4} \mathrm{~cm}^{-1}$ [Tri88].

Using the experimental absorption coefficient, we evaluate the oscillator strength according to the Smakula's relationship [Dea67]:

$$
N^{\prime} f_{X}=0.97 \times 10^{16} \eta \Gamma \alpha_{X}^{\max }
$$

For the 1-s exciton, substituting $\alpha_{X}^{\max }=1.4 \times 10^{4} \mathrm{~cm}^{-1}, \eta=3.6, \Gamma \sim 3 \mathrm{meV}$ and $N^{\prime}=2.2 \times 10^{22} \mathrm{~cm}^{-3}\left(N^{\prime}=1 / v_{0}\right)$ into Eq. 5.18, we have $f_{X} \sim 7 \times 10^{-5}$, which is in accordance with $f_{X}=8 \times 10^{-5}$ evaluated by Eq. 5.10.

\subsection{Extrinsic transitions}

\subsubsection{Acceptor-band recombination $\left(e, A^{0}\right)$}

Eagles and Dumke have treated the $\left(e, A^{0}\right)$ transition quantitatively Eag60, Dum63. In their theory, a model for the acceptor ground state of GaAs was proposed incorporating the following assumptions:

1) a discrete, shallow, non-overlapping acceptor state;

2) a slowly varying hydrogenic envelope function for the bound hole;

3) a valence band density of states unaffected by the presence of many impurities.

With the above assumptions we write out the wave function for the acceptor ground state: 


$$
\Psi_{A}(\boldsymbol{r})=\frac{1}{\sqrt{\pi a_{A}^{3}}} e^{-r / a_{A}} u_{v 0}(\boldsymbol{r})
$$

The Fourier transformation of the envelope wave function given by Eq. 5.19 yields

$$
\frac{1}{\sqrt{\pi a_{A}^{3}}} e^{-r / a_{A}}=\sum_{k} c(\boldsymbol{k}) \exp (\boldsymbol{k} \cdot \boldsymbol{r})
$$

where

$$
c(\boldsymbol{k})=\frac{8 \pi^{1 / 2}}{V_{g}^{1 / 2} a_{A}^{5 / 2}\left(1 / a_{A}^{2}+k^{2}\right)^{2}},
$$

and

$$
a_{A}=\frac{\hbar^{2} \epsilon_{0}}{e^{2} m_{A}}
$$

Similar to the analysis of intrinsic transitions where the oscillator strength is given by $f_{c v}$, here we define the oscillator strength for $\left(e, A^{0}\right)$ transition by calculating Eq. 5.1 with envelope function given by Eq. 5.21. So we have

$$
f_{A}=\frac{v_{A}}{v_{0}} f_{c v}
$$

where $v_{A}$ is defined as

$$
v_{A}=64 \pi a_{A}^{3},
$$

representing the acceptor volume. The sense of so-defined oscillator strength $f_{A}$ for the $\left(e, A^{0}\right)$ transition can be well illustrated in the following analysis of absorption coefficient.

The absorption coefficient due to the no-phonon transition of electron between a conduction state and a hydrogen-like ground state at the acceptor level can be computed by Dum63.

$$
\alpha_{A}(E=\hbar \omega)=\frac{512 \pi e^{2} \hbar\left|p_{c v}\right|_{a v}^{2}}{\eta c m^{2} \hbar \omega E_{A}^{3 / 2}}\left(\frac{m_{e}}{m_{A}}\right)^{3 / 2} \frac{\left(E-E_{0}+E_{A}\right)^{1 / 2}}{(1+t)^{4}} N_{A^{-}},
$$

where $N_{A^{-}}$is ionized accepto concentration, $\left|p_{c v}\right|_{a v}^{2}$ relates to $\left|p_{c v}\right|^{2}$ in Eqs. 5.4 and 5.5 by $4\left|p_{c v}\right|_{a v}^{2}=\left|p_{c v}\right|^{2}$ due to taking four for the degeneracy of acceptor level, and $t$ is given by:

$$
t=\frac{m_{e}}{m_{A}} \frac{E_{l}-E_{0}+E_{A}}{E_{A}}
$$

Here $E_{l}$ is the incident light energy, and $m_{A}$ is the acceptor effective mass. According to the relationship between $a_{A}$ and $m_{A}$ given by Eq. 5.22 , taking $a_{A}=21 \AA$, we have: $m_{A} \simeq 0.31 m$.

If we let $N_{A^{-}}=1 / v_{0}$, i.e., provided that the ionized acceptors have the same density as the e-h pairs in the crystal, we can rewritten the Eq. 5.25 as 


$$
\alpha_{A}(E)=\frac{v_{A}}{v_{0}} \frac{E}{E_{X}^{3 / 2}} \frac{\alpha_{0}}{(1+t)^{4}}\left(E-E_{0}+E_{A}\right)^{1 / 2} \propto f_{A} \frac{\left(E-E_{0}+E_{A}\right)^{1 / 2}}{(1+t)^{4}},
$$

where $\alpha_{0}$ (defined in Eq. 5.12), $v_{A}$ (defined in Eq. 5.24), and the oscillator strength $f_{A}$ (defined in Eq. 5.23) have been used for brevity in writing the equation. Comparing this absorption expression to the absorption expression in Eq. 5.13 for free e-h pairs immediately shows the justification of our definition of oscillator strength $f_{A}$ given by Eq. 5.23 :

$$
\frac{\alpha_{A}}{\alpha_{e-h}}=\frac{f_{A}}{f_{c v}} \frac{\left(E-E_{0}+E_{A}\right)^{1 / 2}}{\left(E-E_{0}\right)^{1 / 2}} \frac{1}{(1+t)^{4}}
$$

Therefore, $f_{A}$ has the meaning analogous to that of $f_{c v}$, both describing the "number" of oscillators, or, the strength of interband transitions.

With $a_{X}=21 \AA$, the evaluation of $f_{A}$ according to Eq. 5.23 yields $f_{c A}=5.9 \times 10^{5}$, much larger than $f_{c v}$. This explains why the absorption of $\left(e, A^{0}\right)$ is comparable with the absorption of e-h pairs though the impurity density is low.

From Eq. 5.25, we can calculate the concentration of acceptor if the absorption coefficient is measured. For the sample 21412, the measured absorption coefficient has been presented in Fig. 4.4 in Chapter 4. Fitting the data using Eq. 5.25 we find that $N_{A^{-}}=6 \times 10^{15} \mathrm{~cm}^{-3}$. See the fitting dots in Fig. 4.4 .

\subsubsection{Acceptor-bound exciton recombination $\left(A^{0}, X\right)$}

The oscillator strength of acceptor bound exciton in GaAs has been studied extensively [Fin86, Hwa73, Osb79, Whi74].

In the acceptor bound exciton there are two holes and one electron bound to a negatively charged acceptor. Because the electron effective mass is generally considerably smaller than the hole effective mass in GaAs, the two holes are bound relatively closer to the acceptor than the electron. Therefore, the electron's wave function is relatively insensitive to the short-range parts of the impurity potential.

The bound exciton wave function can be written as

$$
\Psi_{B X}(\boldsymbol{r}, \boldsymbol{R})=\Phi_{B X}\left(\frac{1}{\sqrt{\pi a_{X}^{3}}} e^{-r / a_{X}}\right) u_{c 0}(\boldsymbol{r}) u_{v 0}(\boldsymbol{r})
$$

where $\Phi_{B X}(\boldsymbol{R})$ is the center-of-mass wave function of the bound exciton. It has been shown that the ratio of the oscillator strength of bound exciton to the oscillator strength of free-exciton is proportional to the ratio of the volume of the center-of-mass wave function to the volume of the primitive cell [Col70], i.e.,

$$
\frac{f_{B X}}{f_{X}}=\frac{\left[\int \Phi_{B X}(R) d^{3} R\right]^{2}}{v_{0}}=\frac{v_{B X}}{v_{0}} .
$$


Substituting Eq. 5.10 into Eq. 5.30, we obtain the oscillator strength

$$
f_{B X}=\frac{v_{B X}}{v_{0}} f_{X}=\frac{v_{B X}}{v_{X}} f_{c v},
$$

where $v_{B X}$ is defined as in Eq. 5.30.

To evaluate the oscillator strength of bound exciton, one has to know $\Phi_{B X}$. Rashba and Gurgenishvili have developed a simple theory to explain the enhancement of the bound exciton oscillator strength over that of free exciton Ras62. In their model (for convenience, we call it R-G model in the following), they simply assumed a $\delta$-function potential imposed on the exciton and neutral impurity. The center-of-mass wave function in such a binding potential has the well-known solution:

$$
\Phi_{B X}(R)=\sqrt{\frac{\kappa}{2 \pi}} \frac{e^{-\kappa R}}{R},
$$

with

$$
\kappa=\frac{\sqrt{2 m_{e}\left|E_{B X}\right|}}{\hbar} .
$$

By inserting Eq. 5.32 into Eq. 5.31, one gets $v_{B X}$, and thus the value of oscillator strength. The R-G model can estimate the magnitude of oscillator strength of bound exciton qualitatively because the potential strength is allowed be adjusted to give the correct binding energy of the bound exciton. For a typical bound exciton, the $f_{B X}$ would be on the order of $10^{3}-10^{5}$ times larger than $f_{X}$. As shown in Section 5.1.2, $f_{X} \simeq 10^{-5}-10^{-3}$, therefore, $f_{B X} \sim 10^{-2}-10^{2}$. For our case: $E_{B X} \simeq 2 \mathrm{meV}$, so we can estimate the radius of the center-of-mass movement and the oscillator strength by the R-G model: $R_{B X}=1 / \kappa \simeq 170 \AA, v_{B X}=8 \pi R_{B E}^{3}$ Col70, thus $f_{B X} \simeq 220$.

The R-G model explains the "giant oscillator strength" of bound exciton in some highpurity semiconductors successfully. However, it is too simple to evaluate the obtained experimental data accurately. The estimated value $f_{B X} \simeq 220$ is too large compared with the experimental observation. A more appropriate estimate in a real system should give a more detailed account of the form of bound exciton wave function.

A general form of a bound exciton state can be expanded in terms of linear combinations of the products of the single-particle Bloch states in the effective-mass approximation, so that [San83]:

$$
\left|\Psi_{B X}\right\rangle=\sum_{\nu_{1}, \nu_{2}, \nu_{3}} \psi\left(\boldsymbol{r}_{1}, \boldsymbol{r}_{2}, \boldsymbol{r}_{3}\right)\left|u_{1}\left(\nu_{1}\right)\right\rangle\left|u_{2}\left(\nu_{2}\right)\right\rangle\left|u_{3}\left(\nu_{3}\right)\right\rangle
$$

where the subscripts 1 and 2 refer to the holes (electrons) in the acceptor (donor) system, and 3 refers to the electron (hole); $u_{i}\left(\nu_{i}\right)(i=1,2,3)$ denotes the periodic part of a Bloch state at the band extremum with spin component $\nu_{i}$. For the conduction band $\nu_{i}=$ $-1 / 2,1 / 2$ and for the valence band $\nu_{i}=-3 / 2,-1 / 2,1 / 2,3 / 2$ in the limit of strong spinorbit interaction, as mentioned in the section 2.1 in Chapter 2. $\psi\left(\boldsymbol{r}_{1}, \boldsymbol{r}_{2}, \boldsymbol{r}_{3}\right)$ is the envelope function, which is the eigen-state of the Hamiltonian (in atomic units: $e=\hbar=1, e^{2} / \hbar c=$ 1/137): 


$$
H=-\nabla_{1}^{2}-\nabla_{2}^{2}-\sigma \nabla_{3}^{2}-\frac{2}{r_{1}}-\frac{2}{r_{2}}+\frac{2}{r_{3}}+\frac{2}{r_{12}}-\frac{2}{r_{13}}-\frac{2}{r_{23}} .
$$

where $\sigma$ is the effective-mass ratio of electron to hole.

For the acceptor bound exciton in GaAs, there are two holes 1 and 2, and one electron 3 bound around the acceptor.

To obtain the envelope function $\psi$, Osbourn and Smith assumed a single-particle product envelope wave function in the acceptor-bound exciton regime Osb79]:

$$
\psi\left(\boldsymbol{r}_{1}, \boldsymbol{r}_{2}, \boldsymbol{r}_{3}\right)=\psi_{0} e^{-\alpha \boldsymbol{r}_{1}} e^{-\alpha \boldsymbol{r}_{2}} e^{-\gamma \boldsymbol{r}_{3}}
$$

where the coefficients $\alpha$ and $\gamma$ are determined by the Ritz variational method of minimizing the expectation value of the Hamiltonian. $\psi_{0}$ is the normalization constant.

Obviously, the radial correlation between the two holes has been neglected in the tentative wave function in Eq. 5.36. So further improvement upon the above calculation was achieved by Sanders and Chang San83. They took correlation effects into account by using wave function which also depends on inter-hole separations and includes Pauli exchange of the two holes. The improved variational wave function has the following form:

$$
\psi\left(\boldsymbol{r}_{1}, \boldsymbol{r}_{2}, \boldsymbol{r}_{3}\right)=F_{0}\left(e^{-\alpha_{1} r_{1}} e^{-\alpha_{2} r_{2}}+e^{-\alpha_{1} r_{2}} e^{-\alpha_{2} r_{1}}\right) e^{-\alpha_{3} r_{3}}
$$

From Eq. 5.37, the center-of-mass motion $\Phi_{B X}(R)$ can be expressed explicitly as

$$
\Phi_{B X}(R) \propto R^{\tau} e^{-\xi R} .
$$

For acceptor bound exciton in GaAs, they got $\tau \simeq 2$ and $\xi \simeq 1.3 / r_{D}$, whence:

$$
\frac{\left[\int \Phi_{B X}(R) d^{3} R\right]^{2}}{v_{X}}=|\langle|I| F\rangle|^{2}=0.54 .
$$

According to Eq. 5.31, the oscillator strength of acceptor bound exciton is thus estimated: $f_{B X}=7.8$.

The absorption coefficient maximum can be deduced in the same way as Eq. 5.17 for the 1-s free exciton, though it should be corrected by the oscillator strength of bound exciton $f_{B X}$ and the acceptor concentration $N_{A}$. So we have

$$
\alpha_{B X}^{\max }=8 \alpha_{0} \frac{E^{\prime}}{\Gamma_{B X}} N_{A} v_{B X}
$$

where $E^{\prime}=E_{0}-E_{X}-E_{B X}$. If we take $\Gamma_{B X} \sim 1 \mathrm{meV}, v_{B X}=0.54 v_{X}, N_{A}=6 \times 10^{15} \mathrm{~cm}^{-3}$, we obtain $\alpha_{B X}^{\max } \sim 500 \mathrm{~cm}^{-1}$. This value is consistent with experimental observations. (See Fig. 4.4: $\alpha_{B X}(1.510 \mathrm{eV}) \sim 150 \mathrm{~cm}^{-1}$; assuming $\Gamma_{B X} \sim 1 \mathrm{meV}$, we deduce that $\alpha_{B X}^{\max } \sim$ $500 \mathrm{~cm}^{-1}$.) 


\subsubsection{Pair luminescence $\left(D^{0}, A^{0}\right)$}

The donor-acceptor pair (DAP) transition is strong as illustrated in the PL spectrum (see Fig. 4.1). In this part we discuss in detail the characteristics of the DAP luminescence.

The DAP luminescence stems from radiative recombination in GaAs of holes and electrons trapped at acceptors and donors which are distributed randomly in the crystal. It is called pair-band or pair luminescence in the literature. Such pair band spectra have been studied extensively in many semiconductor compounds in the past [Lei67, Ros70, Tho64, Tho65, Vin73].

The emission energy of DAP luminescence depends on the spatial separation between the donor and acceptor in a pair. In the initial state from which the emission starts, an electron is located at the donor level, while a hole locates at the acceptor level, thus the energy of initial state is (the energy origin is assigned at the position of the acceptor level)

$$
E_{i}=E_{0}-\left(E_{D}+E_{A}\right) .
$$

In the final state, both the donor and acceptor are ionized, leaving the effective charges. Due to the Coulomb attractive interaction, there is $-e^{2} / \epsilon R$ left for the final state. So the emission energy $E_{\text {lum }}$ is blue-shifted:

$$
E_{\text {lum }}=E_{0}-\left(E_{D}+E_{A}\right)+e^{2} / \epsilon R_{p},
$$

where $R_{p}$ is the separation between the pair.

From Eq. 5.42 it can be seen that $E_{\text {lum }}$ shifts towards higher energies with decrease of $R_{p}$. Since the values of $R_{p}$ in crystals are discrete, the PL spectra of DAP should be composed of numerous lines. However in GaAs, they are too close to be resolved.

The transition probability of DAP also depends on $R_{p}$. The probability is proportional to the square of the overlap of the donor and acceptor wave functions [Tho64, Tho65]. Assuming hydrogen-like wave function for the donor and acceptor, we have the Bohr radius for acceptor $a_{A} \approx 21 \AA$, and for donor $a_{D} \approx 100 \AA$ in our GaAs sample 21412. Hence, the spread of the wave function of a donor is generally much larger than that of an acceptor. With a good approximation, we consider the spread of the donor wave function only, so that the transition rate is given by

$$
W=W_{0} \exp \left(-2 R_{p} / a_{D}\right)
$$

For the case with the pair concentration about $6 \times 10^{15} \mathrm{~cm}^{-3}$, the mean separation between the pairs is about $600 \AA$. For such a concentration, the DAP recombination rate is typically about $10^{7} \mathrm{~s}^{-1}$ Kam76, comparable to the recombination rate of $\left(e, A^{0}\right)$ (in order of $\mu \mathrm{s}$ Dum63]). We see that both $\left(D^{0}, A^{0}\right)$ and $\left(e, A^{0}\right)$ are strong in the PL experiment. The Coulomb potential for the mean distance $600 \AA$ is about $2 \mathrm{meV}$. It seems that the emission peak of $\left(D^{0}, A^{0}\right)$ would be shifted $2 \mathrm{meV}$ higher than that for $R_{p} \rightarrow \infty$, i.e., $1.4890+0.002=1.4910(\mathrm{eV})$. In the experiment, however, the peak position is found to be $1.4915 \mathrm{eV}$, a little higher than $1.4910 \mathrm{eV}$. This is just because more "isolated" DAP are excited in the crystal under the high-intensity excitation condition. The distant 
pairs become saturated and extra holes and electrons will be forced to find the less likely, more closed spaced pairs. The oscillator strength increases with the $R_{p}$ exponentially. Consequently, the DAP luminescence is shifted to higher energy.

As the exciting light intensity is reduced, closer pairs are not significantly excited so that more non-isolated pairs will be able to radiate sufficient energy. Presumably at lowest excitation intensity, one can approach the pair for infinitely distant pair, so obtain the low energy limit of the pair luminescence [Ulb73, Ulb78]. 


\section{Chapter 6}

\section{Impurity-induced RRS below the $E_{0}$ band gap: results and discussions}

This chapter treats the impurity-induced RRS. We first present the experimental results. Then we attempt to interpret the impurity-induced RRS data, i.e.: the resonant firstorder scattering at the threshold of $\left(e, A^{0}\right)$; multi-phonon scattering above the threshold of $\left(e, A^{0}\right)$; and the resonant scattering at $\left(A^{0}, X\right)$.

\subsection{RRS spectra}

The laser was tuned from $1.46 \mathrm{eV}$ to $1.52 \mathrm{eV}$ for the purpose of this study. A series of RRS spectra at different excitation energy were recorded and presented in Figs. 6.1 6.4. In the following, unless a special note is added, the spectra are exhibited on the absolute energy scale in unit $\mathrm{eV}$. the advantage of using such a scale is to identify the impurity luminescence and its replicas clearly because they have more or less fixed energy positions in the spectra. In all the recorded spectra, a broader line at $1.454 \mathrm{eV}$ can be identified, which is attributed to the 1-LO phonon replicas of the impurity luminescence, most of which originates from $\left(D^{0}, A^{0}\right)$. A sharp peak which has a Stokes shift of $36.5 \mathrm{meV}$ with respect to the excitation energy appears in all the spectra, too. This line is simply due to the normal Raman scattering.

Several other spectral features are notable in different excitation energy regions:

Fig. 6.1 illustrates the RRS spectra with excitation energy below the $\left(e, A^{0}\right)$ energy level. The phonon replica signal is apparently identified in the spectra, though weak because it originates from up-converted luminescence which will be explained in Chapter 7. The intensity ratio of LO peak to TO peak changes drastically with laser tuning. It is also observed that the replica is slightly suppressed when the excitation energy approaches $\left(D^{0}, A^{0}\right)$ recombination.

Fig. 6.2 and Fig. 6.3 show the spectra with laser energy very close to or higher than $\left(e, A^{0}\right)$ transition energy level. When the excitation energy is raised to the level which is higher than the energy level of $\left(e, A^{0}\right)$, the linewidth of the Raman scattering peak increases. 


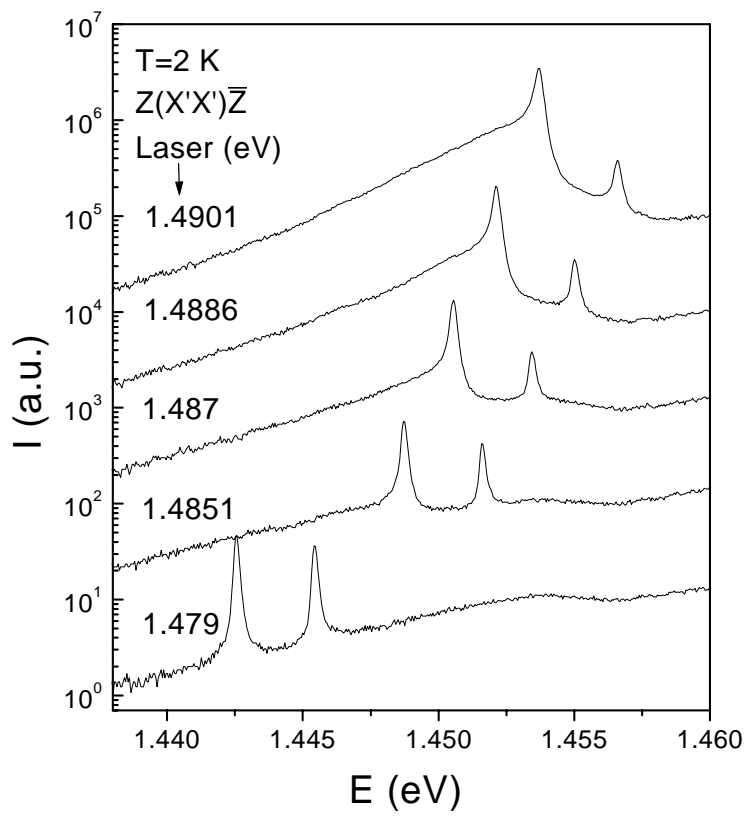

Figure 6.1: RRS spectra with excitation energy below the $\left(e, A^{0}\right)$ threshold.

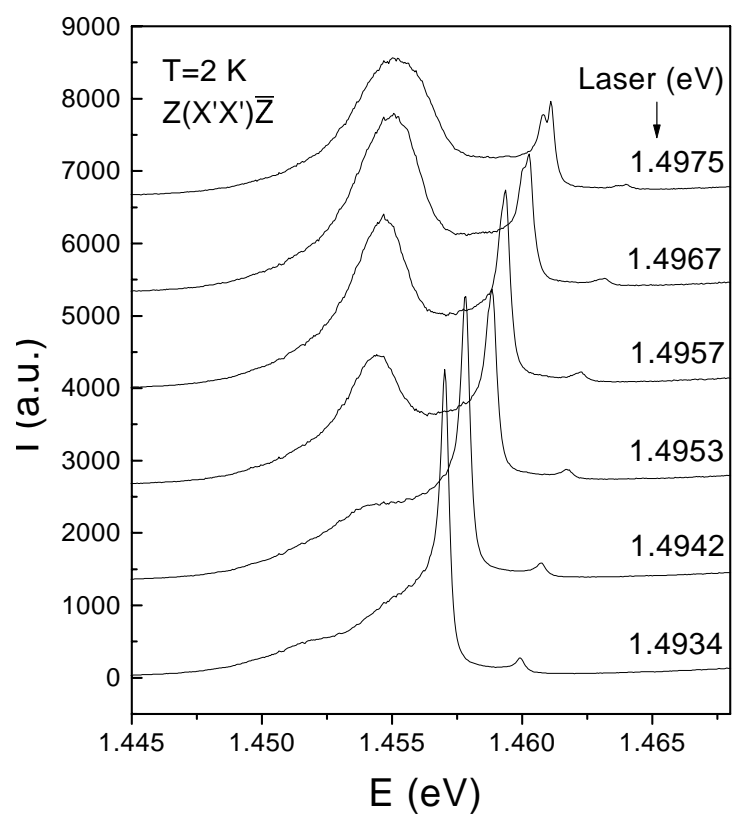

Figure 6.2: RRS spectra with excitation energy in the vicinity of the $\left(e, A^{0}\right)$ threshold.

With laser energy continuously raised, the "splitting" of this line is observed evidently. The "splitting" increases with excitation energy. One of the "splitted" line is still ascribed to 


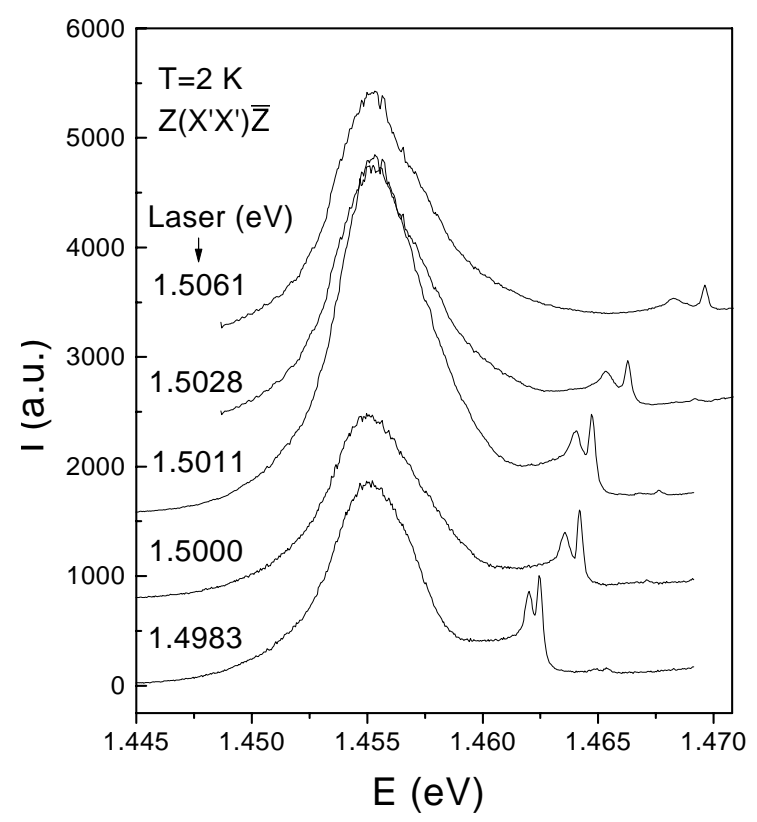

Figure 6.3: RRS spectra with excitation energy above the $\left(e, A^{0}\right)$ threshold.

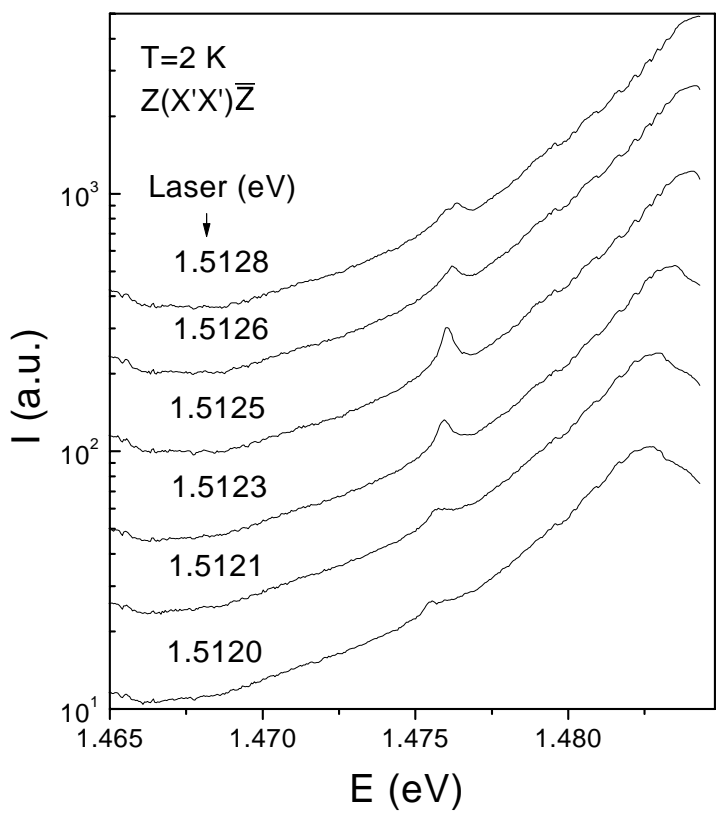

Figure 6.4: RRS spectra with excitation energy around $\left(A^{0}, X\right)$.

the normal Raman scattering because it has the fixed Stokes shift $36.5 \mathrm{meV}$. The other line, however, has a larger Stokes shift, which is, in fact, due to additional scattering processes. 
For the intensity observation, the LO signal increases when the laser is approaching $\left(e, A^{0}\right)$ then it decreases when the laser is above the $\left(e, A^{0}\right)$ threshold. We also find that the RRS signal overlaps the phonon replica signal seriously when the incident light has the energy to excite $\left(D^{0}, A^{0}\right)$ or approach the $\left(e, A^{0}\right)$ threshold; and when the overlapping happens, the RRS line shape is not symmetrical at all. This spectral feature relates to impurity spatial distribution effect and will be discussed in Chapter 7. When the laser energy is raised, the RRS signal becomes separate from the replica signal obviously. The intensity of the phonon replica signal also shows increase and decreases when continuously raising the laser energy, so there exists a maximum around $1.50 \mathrm{eV}$ for this replica signal.

Fig. 6.4 shows the case when the sample was excited at the energy around 1.5125 $\mathrm{eV}$. The normal Raman scattering signal becomes stronger again, reaching its intensity maximum exactly at $1.5125 \mathrm{eV}$. This energy just corresponds the transition energy of $\left(A^{0}, X\right)$. So it shows Raman scattering resonance at $\left(A^{0}, X\right)$

\subsection{First-order RRS at the threshold of $\left(e, A^{0}\right)$}

As mentioned in the introduction part of the thesis, for the direct gap semiconductors, the intrinsic RRS study near the $E_{0}$ gap is hampered by the appearance of strong luminescence. Since impurity-related optical transitions show dominance in photon emission and absorption processes at low temperature, we expect that the Raman polarizability gains significant contribution from the impurity-induced scattering, keeping in mind that Raman scattering is a third-order process including the optical transitions twice. In this section, we will show clearly that the impurity-induced RRS occurs at the threshold of the $\left(e, A^{0}\right)$, and the scattering efficiency is not negligible at liquid helium temperature. In the back-scattering configurations, both the LO and TO scattering have been observed; however, the intensity ratio LO/TO increases drastically up to the $\left(e, A^{0}\right)$ threshold, implying that the Fröhlich interaction plays an important role. We will analyze the large scattering intensities of both the LO and TO scattering. This is, to our knowledge, the first confirmation and analysis of the phenomenon.

At first we would like to review some important aspects for the intrinsic RRS.

As explained in Chapter 2, there are two mechanisms for first-order optical phonon Raman scattering: one is due to the deformation potential (DP); the other is the Fröhlich interaction. The Fröhlich interaction can be further classified into the intraband Fröhlich (F) and interband Fröhlich interaction, with the latter also called as electric-optic (EO) interaction. The TO scattering occurs exclusively through the DP interaction, whereas the Raman scattering by LO phonons can result from both the DP and Fröhlich interactions. In the dipole-approximation (the limit in which the phonon wave vector $\boldsymbol{q}$ vanishes), however, only the DP and EO mechanisms contribute to the LO scattering, which is thus called dipole-allowed scattering. The intraband Fröhlich matrix elements do not produce LO Raman scattering when the phonon wave number $q$ approaches zero. For this reason, the F-induced LO scattering is also called dipole-forbidden scattering.

In the back-scattering geometry, only LO scattering is allowed for $T_{d^{-}}$-symmetry crystals. 
The selection rules for the dipole-allowed scattering are determined by the symmetry of the $\boldsymbol{k}=\mathbf{0}$ phonons. Previous investigations have shown that the $\Gamma_{15}$-symmetry phonons are mostly responsible for the Raman scattering in GaAs, and the configuration for observing the scattering by this type of phonons can be $Z(X Y) \bar{Z}$ according to the selection rules.

For the intraband F-induced Raman scattering, one has different selections rules. Previous studies showed that the Raman tensor was diagonal in GaAs at resonance [Men85. However, because the scattering depends on the value of $q$, strictly, the symmetry of the scattered phonon cannot be analyzed using point-group theory. This dipole-forbidden scattering may have a large cross-section near resonance.

Then we consider the impurity-induced Raman scattering occurring at the threshold of $\left(e, A^{0}\right)$.

As explained in the last chapter, $\left(e, A^{0}\right)$ is the recombination between a free electron in a conduction band state and a bound hole at the shallow acceptor level. The Raman scattering at $\left(e, A^{0}\right)$ includes the interband optical transition twice. In fact, the actual scattering is a four- or more-step process, where higher-order perturbation theory should be employed. In the scattering, there is electron-impurity interaction which plays an important role in the breakdown of the selection rules and the large scattering cross-sections Gog76. The process can be expressed by Feynman diagram as shown in Fig. 6.5 [Men85, where the impurity-electron interaction vertex is represented by a solid triangle. Here we don't consider the concrete mechanism for the impurity-electron interaction. It has been shown that the scattering is independent of the rate of the electron-impurity interaction Gog76. Take this point as an assumption in our analysis, therefore, we can still regard the scattering as an equivalent third-order process, but we include the effects which this impurity-electron interaction brings: $\boldsymbol{q}$-relaxation and "depolarization"; reinforcement of the $q$-dependent intraband F-scattering.

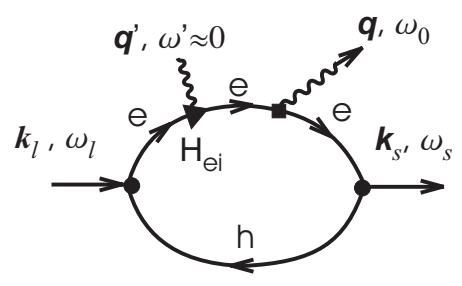

Figure 6.5: The Feyman diagram including electron-impurity interaction, whose vertex is labeled by a solid triangle. The electron-impurity interaction gives rise to a quasi-elastic scattering where the energy change may be negligible: $\hbar \omega^{\prime} \approx 0$. But the value $q^{\prime}$ may be large in the momentum transfer induced by the interaction. The definitions of other symbols have been explained in Fig. 2.6 .

For the bound state involved in scattering, in the hydrogenic model, its wave function (localized at the acceptor site) has been given by Eq. 5.19. Because normally $a_{A}$ is small for acceptors (for carbon-acceptor $a_{A}$ is about $21 \AA$ with a binding energy $E_{A}$ about 27 $\mathrm{meV}$, see Appendix A), considering that the acceptors are distributed in the real space, 
we see that the acceptor wave functions are little overlapping with each other compared to the relatively big distance among the acceptors (for acceptor density $6 \times 10^{15} \mathrm{~cm}^{-3}$, the average separation is about $600 \AA$ ). So the scattering is localized. On the other hand, the small $a_{A}$ leads to a quite "flat" distribution of $c(\boldsymbol{k})$ in $\boldsymbol{k}$-space (for the half maximum of $c(\boldsymbol{k}),\left|\boldsymbol{k}_{1 / 2}\right| \sim 1 / a_{A} \sim 4 \cdot 10^{6} \mathrm{~cm}^{-1}$, much larger than $\left.\left|\boldsymbol{k}_{l}-\boldsymbol{k}_{s}\right| \sim 2 \cdot 10^{4} \mathrm{~cm}^{-1}\right)$. The distribution $|c(\boldsymbol{k})|^{2}$ just corresponds to the population of acceptors at a definite $\boldsymbol{k}$. For optical transitions, according to Fermi's Golden Rule, the matrix element is determined by $<\phi_{f}\left(\boldsymbol{k}^{\prime}\right)\left|H_{E R}\right| \phi_{i}(\boldsymbol{k})>$ in the $\boldsymbol{k}$-space. For a transition with energy $E$, a corresponding $\boldsymbol{k}$ is definitely chosen so that only the $c(\boldsymbol{k})$ part of the acceptor wave function contributes to the transition. We thus assume that the assembly of acceptors are equivalent to an assembly of particles in the free valence states which have the same energy but different eigen-momenta $\boldsymbol{k}^{\prime}$ s: an energy degenerate "acceptor-valence-band". Such a viewpoint has been supported by the Dumke's $\left(e, A^{0}\right)$ emission (absorption) analysis, where the problem was solved by calculating the recombination between an acceptor-bound hole, virtually with $-\boldsymbol{k}_{h}$, with a free electron with real $\boldsymbol{k}_{e}$. We will show later in this section that our absorption measurement at $2 \mathrm{~K}$ is consistent with this viewpoint experimentally. The advantage of such an interpretation of the real physical process lies on its facilitating our analysis of impurity-induced scattering.

The absorption is a first-order quantum process, whereas the treated Raman scattering here is a third- or higher-order process. However, it includes the bound-to-free optical transitions twice, and in each of the radiative processes, we can use such an "acceptorvalence-band" picture. Hence, we can refer the extrinsic scattering problem to the treatment of intrinsic scattering directly. Of course, some modifications should be made:

The first modification is just that we shall replace $E_{0}$ by $\left(E_{0}-E_{A}\right)$ in the RRS formulas which are obtained near the $E_{0}$ gap in Chapter 2. So the case is just like shifting a valence band upwards by $E_{A}$. This is easily understood because we are discussing the extrinsic scattering near $\left(e, A^{0}\right)$ instead of $E_{0}$.

The second modification stems from the difference of oscillator strength between the $\left(e, A^{0}\right)$ transition and transition of free e-h pairs. If the oscillator strength of free e-h pairs per unit cell is represented by $f_{c v}$ in Eq. 5.3, the oscillator strength of $\left(e, A^{0}\right)$ is given by $f_{c A}$ in Eq. 5.23.

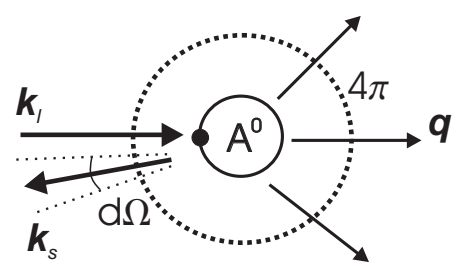

Figure 6.6: The propagation of the scattered phonons is not kinetically decided by the wave vectors of the incident and scattered photons in the impurity-induced scattering. The polarizations of the light and the phonons are not certain, either (not depicted). 
Finally, the selection rules are revised. As mentioned in the foregoing discussion, although we regard that many acceptors form a "acceptor-valence-band", in fact, their wave functions are localized. In such a case, $\boldsymbol{k}$ is actually not a good quantum number for the bound state, and the eigen-state of acceptor is just the sum of free states with different $\boldsymbol{k}$ 's whose weight factors are determined by $c(\boldsymbol{k})$. So in the impurity-induced Raman scattering, the electronic momentum in the final state can be different from that in the initial state, and this momentum difference is compensated by the scattered phonons, and possibly, also by the movement of the acceptor nuclei. So the momentum of the scattered phonon is not decided kinetically by the incident and scattered photons any longer. This effect is called $\boldsymbol{q}$-relaxation, and is the key to understand the breakdown of the selection rules in the impurity-induced Raman scattering. Notice that the polarization of the scattered photon is not certain, either, just because of the impurity-electron interaction in the scattering. Moreover, if large momentum transfers happen in the impurity-induced scattering, the selection rules deduced for $\boldsymbol{q}=\mathbf{0}$ symmetry do not hold any more. And this may also enhance the forbidden F-induced scattering at resonant excitation condition. So the selection rules would be broken not only because the symmetry of the scattering geometry is not satisfied due to the uncertainty of the orientation and the polarization of the wave vectors of the phonons, but also because the scattering could induce forbidden scattering by phonons with large $q$ 's.

We will show that the observed phenomenon can be understood by the above considerations.

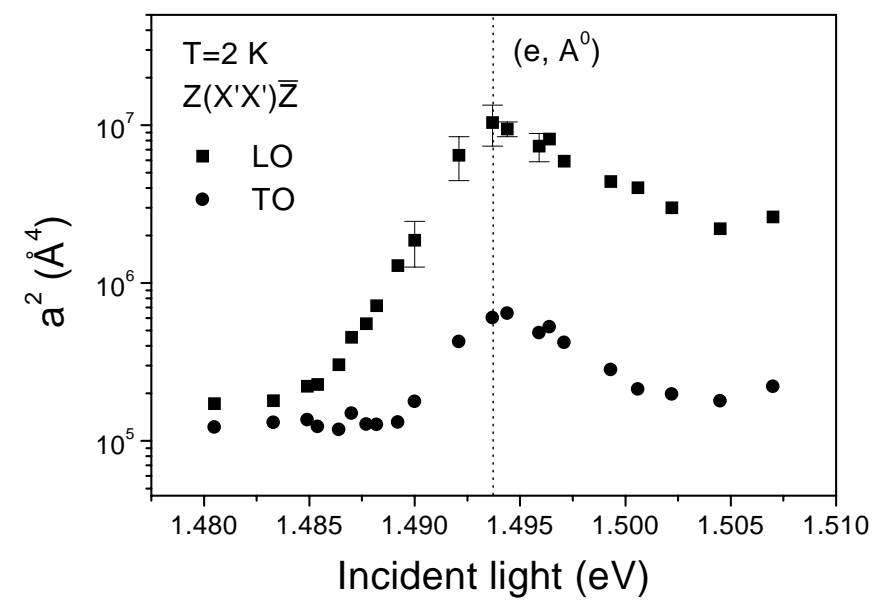

Figure 6.7: The Raman intensities of the LO and TO scattering. The resonance at the $\left(e, A^{0}\right)$ threshold is evidently shown. The selection rules do not hold for the impurity-induced scattering. The errors (see the error bars) come from the overlapping of the RRS signal with the luminescence phonon replica signals.

Fig. 6.7 presents the Raman intensities of scattering by LO and TO phonons in the $Z\left(X^{\prime} X^{\prime}\right) \bar{Z}$ configuration. As mentioned in the preceding section, only the LO scattering 
can be observed in this configuration for intrinsic scattering. However, we observed both the TO and LO scattering lines in the experiment, so the selection rule is broken. Notice that although we did not perform stringent back-scattering measurement because the incident light was striking on the surface of the sample at a small angle, the impinging angle inside the crystal was less than $5^{\circ}$ due to the large refractive index of GaAs.

In Fig. 6.7, we can identify the resonance at the threshold of $\left(e, A^{0}\right)$ apparently. The maximum at $1.4944 \mathrm{eV}$ is consistent with its peak position of the $\left(e, A^{0}\right)$ emission in the photo-luminescence measurement. The LO scattering shows a much stronger resonance than the TO scattering. The scattering intensity ratio LO/TO is not constant.

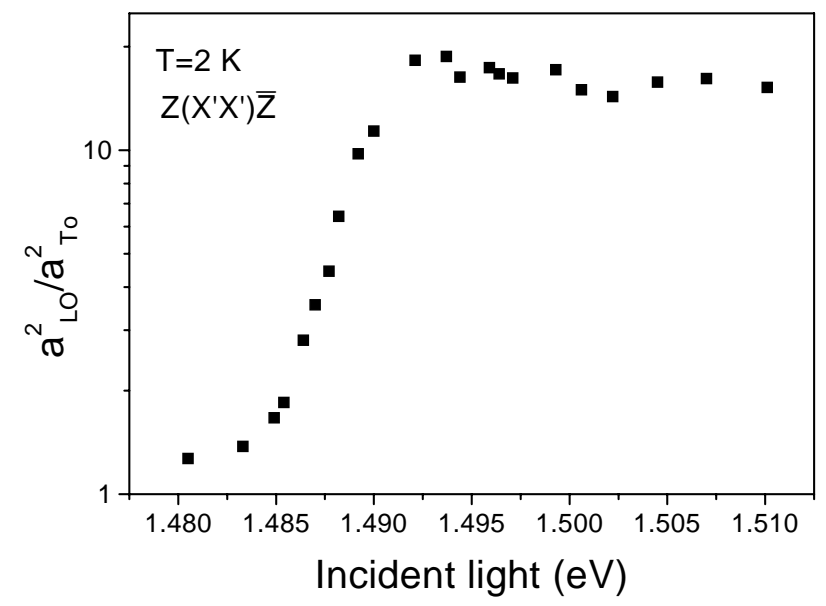

Figure 6.8: The Raman intensity ratio LO/TO changes dramatically near resonance, implying that the intraband Fröhlich interaction plays an important role in the LO scattering.

Fig. 6.8 illustrates the $\mathrm{LO} / \mathrm{TO}$ scattering intensity ratio. The ratio increases drastically from $1.475 \mathrm{eV}$ to $1.503 \mathrm{eV}$, then keeps almost constant above $1.503 \mathrm{eV}$. We know that the TO scattering is solely owing to the DP mechanism, while the LO scattering can result from all the mentioned mechanisms. Provided that there were no contribution from the intraband Fröhlich interaction, we would expect that the LO/TO intensity ratio would be more or less constant because it is then determined by the Faust-Henry coefficient which is almost constant. One may argue that in the impurity-induced scattering, the scattering geometry is not certain because the $\boldsymbol{q}$ of the scattered phonons has an orientation distribution, but regarding that EO and DP interactions are active on the phonons of the same symmetry and thus of the same origin, the uncertainty of the scattering geometry only changes the magnitude instead of constancy of the ratio.

Therefore the enormous enhancement of the LO scattering in contrast to the TO scattering simply indicates that intraband-Fröhlich interaction plays an important role in the impurity-induced scattering.

Next, we try to interpret the DP- and F-induced scattering in a quantitative way. 
Since the TO scattering is uniquely active through the DP mechanism, for the DPinduced scattering, we can utilize the TO data given in Fig. 6.7. In Fig. 6.9, we re-plot the TO data, and show our simulation results as well. Notice that here the TO Raman intensity is given in arbitrary units because of the geometry uncertainty in the impurityinduced scattering. Also notice that the presented data exclude the intrinsic scattering contribution because in the applied configuration the intrinsic TO scattering is forbidden.

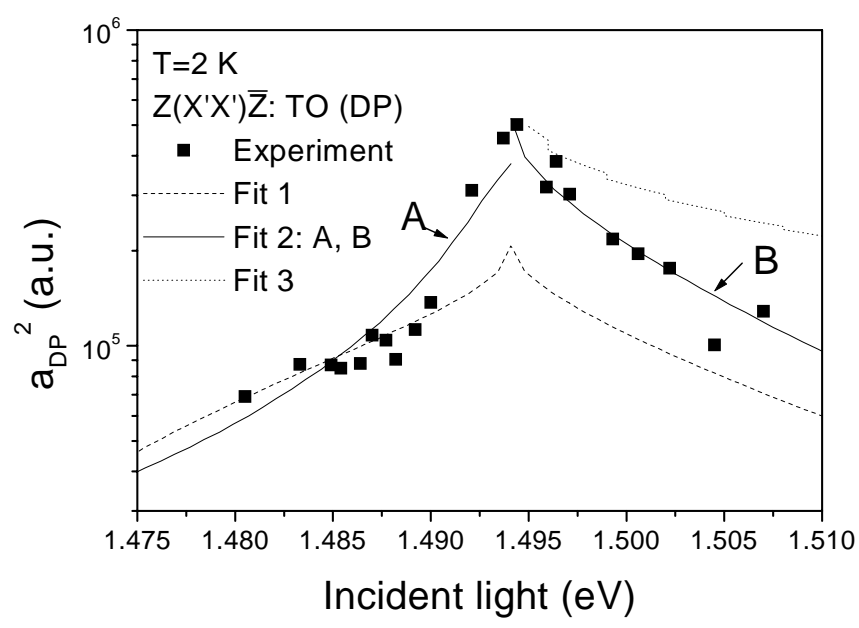

Figure 6.9: The TO scattering due to the DP mechanism. The scattering intensity is plotted in arbitrary units. The dashed line "Fit-1" is simulation according to Eq. 2.25; the solid line "Fit-2" is the fitting with " $\mathrm{A}$ " for the scattering below the $\left(e, A^{0}\right)$ threshold, " $\mathrm{B}$ " the fitting above the $\left(e, A^{0}\right)$ threshold, where the denominator $(1+t)^{8}$ has been included. The dotted line "Fit-3" is the fitting without such a denominator.

The dashed line "Fit-1" comes from the application of Eq. 2.25 to the scattering at the $\left(e, A^{0}\right)$ threshold. We see that it cannot reproduce well the experimental result on that if we try to fit the data below the $\left(e, A^{0}\right)$ threshold, the calculated Raman intensity above the threshold is smaller than that from the experiment; or, on the other hand, if we try to fit the data above the $\left(e, A^{0}\right)$ threshold, the measured Raman intensity shows a more rapid increase with the incident energy than that from the simulation, see the solid line "Fit-2".

To understand this sharpness of resonance at the $\left(e, A^{0}\right)$ threshold, we think that the Coulomb interaction between the involved electron and the hole, so-called "exciton effect" may play a roll. It is well known that the "exciton effect" can enhance greatly the scattering cross-section [Mar71b], and for intrinsic scattering near $E_{0}$ in GaAs at low temperature the enhancement by "exciton effect" is fairly large [Soo87. So we take this effect into account in understanding the steeper increase of scattering intensity below the $\left(e, A^{0}\right)$ threshold. In a heuristic and tentative way, we fit the points by using the following function [Soo87]:

$$
\left|\hat{\mathbf{e}}_{s} \cdot \mathbf{R}(q) \cdot \hat{\mathbf{e}}_{l}\right|^{2} \propto\left|\ln \left[\frac{E_{L}-E_{0}+i \Gamma}{E_{L}-E_{0}-\hbar \omega_{0}+i \Gamma}\right]\right|^{2} /(1+t)^{8}
$$


and the fitting line is plotted in Fig. 6.9 as "Fit-2B", showing a more intense scattering than the one without inclusion of "exciton effect". Notice that the denominator $(1+t)^{8}$ is added in Eq. 6.1, which comes from Eq. 5.27 or 5.28 for the $\left(e, A^{0}\right)$ transiton.

However, in the fitting, we find we should use different coefficients for the scattering below the threshold and above the threshold, and the fitting coefficient above the threshold is about 3 times bigger than the the fitting coefficient below the threshold. We try to understand this by consider the influence of momentum transfer, i.e., the $q$-relaxation effect in the scattering.

From the standpoint explained at the beginning of this section, we have seen that in the "acceptor-valence-band" picture when the excitation above the $\left(e, A^{0}\right)$ threshold, the real transition happens and there exists large momentum transfer by electrons (holes), so the $q$-relaxation effect is obviously significant. Below the $\left(e, A^{0}\right)$ threshold, however, there happens no real optical transition: the intermediate state for the Raman scattering is a virtual state. This means: the momentum of the final electronic state would fairly probably keep the same as that of the initial state, so the momentum transfer is small. So the scattering below the $\left(e, A^{0}\right)$ threshold has smaller $q$-relaxation effect than the scattering above the $\left(e, A^{0}\right)$ threshold. In this way, the Raman intensity factor 3 for scattering above the $\left(e, A^{0}\right)$ threshold is thus understood.

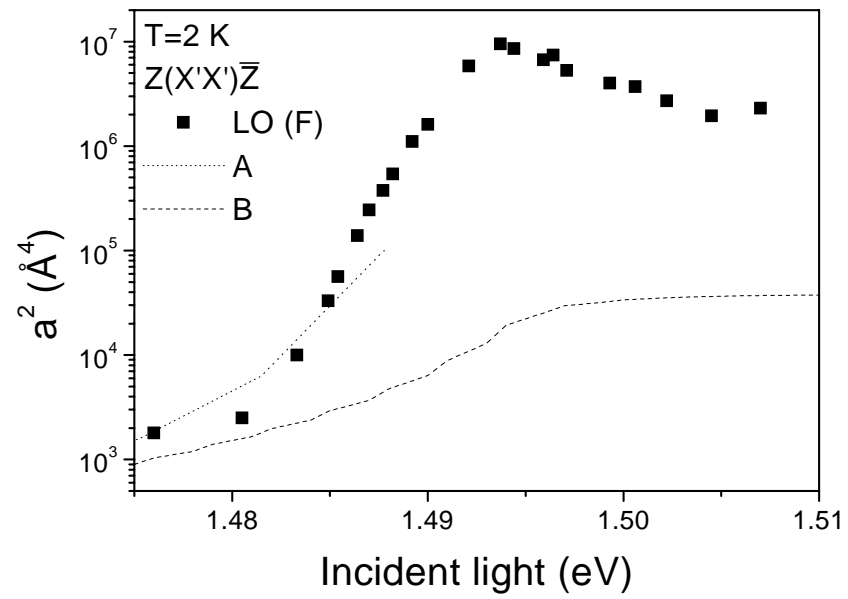

Figure 6.10: The F-induced LO scattering. The dotted line "A" is obtained from Eq. 2.27, the dashed line "B" refers to the calculation in Refs. Bec86, Soo87] which has considered the Coulomb interaction between the scattered e-h pairs.

The curve "Fit-3" is drawn without correction of the denominator $(1+t)^{8}$, which appears in Eq. 5.27 above the $\left(e, A^{0}\right)$ threshold. Comparing it to the curve "Fit-2" which has taken account of this denominator, i.e., the $\boldsymbol{k}$-dependence of oscillator strength, we see that the "Fit-2" is more satisfactory for the fitting. This is also an indication that the theory for uncorrelated e-h pairs is helpful for the understanding of the scattering above the $\left(e, A^{0}\right)$ threshold. 
Finally, we deal with the F-induced LO scattering. With the assumption that the $(\mathrm{DP}+\mathrm{EO})$-contributed LO scattering has a constant ratio with respect to the TO scattering, we simply subtract this part of contribution by subtracting the TO scattering intensity multiplied by an adjustable factor. The rest contribution is ascribed to the F-mechanism, and is plotted in Fig. 6.10. We have noticed that in the applied configuration, both the (DP+EO)-scattering and F-scattering exist according to the selection rule for the intrinsic scattering. However, because no interference effect [Men85] has been essentially detected for our extrinsic scattering, we subtract the (DP+EO)-induced Raman intensity $a^{2}$ instead of using amplitude $a$ for data treatment. Notice that here the presented LO data in the applied configuration include intrinsic scattering contributions. However, this intrinsic contribution is relatively small and constant as a background within the studied energy region, so we neglect the intrinsic contribution in the analysis.

In Fig. 6.10, the dashed line "A" is calculated according to Eq. 2.27, with its absolute value of the scattering intensity referred to the intrinsic scattering data of GaAs at $77 \mathrm{~K}$. We see that the F-induced scattering has a very large cross section although the impurity concentration is low. This is mainly due to the large oscillator strength of the $\left(e, A^{0}\right)$ transition.

As calculated in Chapter 5, $f_{c v}$ is 14.5 according to Eq. 5.3 and $f_{c A}$ is $5.9 \times 10^{5}$ according to Eq. 5.23. This large oscillator strength has actually been confirmed in the absorption measurement. With the acceptor concentration taken into account, the absorption coefficient ratio at the $\left(e, A^{0}\right)$ threshold is estimated as $\alpha_{A} / \alpha_{e-h}=f_{A} N_{A^{-}} / f_{c v} N^{\prime}=v_{A} N_{A^{-}} \sim$ $10^{-2}\left(N^{\prime}=1 / v_{0}\right)$. In fact, comparing our absorption measurement at the $\left(e, A^{0}\right)$ threshold to the intrinsic absorption above the band gap $E_{0}$, we find the evaluation is reasonable: $1 \%$ above the $\left(e, A^{0}\right)$ edge, we have $\alpha_{A} \sim 10 \mathrm{~cm}^{-1}$; the absorption coefficient is relatively constant above the band gap with $8 \times 10^{3} \mathrm{~cm}^{-1}$ [Tri88], where the absorption from continuum exciton is about $2 \pi$ times bigger that from free e-h pairs Pey93, so the absorption from uncorrelated free e-h pairs is deduced to be about $10^{3} \mathrm{~cm}^{-1}$ when the excitation is $1 \%$ above the $E_{0}$ gap. So we also have $\alpha_{A} / \alpha_{e-h} \sim 10^{-2}$. Although impurity density is low, the impurity absorption is comparable to the absorption from free electron-hole pairs. This is just due to the large oscillator strength of the $\left(e, A^{0}\right)$.

For the Raman scattering, an inspection of Raman tensor, which can be obtained by measuring scattering rate $S^{\prime}$ according to Eq. 2.36, shows that

$$
\frac{a_{c A}^{2}}{a^{2}} \propto \frac{f_{c A}^{2} N_{A^{-}}}{f_{c v}^{2} N^{\prime}}=\frac{f_{c A}^{2} N_{A^{-}} v_{0}}{f_{c v}^{2}}
$$

From Eq. 6.2 we estimate that the impurity-induced scattering is about 450 times stronger than the intrinsic scattering of free e-h pairs. Actually, we find this value is in agreement with the ratio obtained from the experiment (about 350-450 around the scattering intensity maximum), though possibly partly fortuitous for this good agreement.

For the F-induced scattering, here again we cannot explain the sharpness of the resonance below the $\left(e, A^{0}\right)$ if we would not introduce other factors which could influence the scattering. So, with the same reason as for DP-induced scattering, we think "exciton effect" may exist in the F-induced scattering below the $\left(e, A^{0}\right)$ threshold, too, giving an 
enhancement of the scattering. The fitting line "B" is referred to the calculation including "exciton effect" given in [Bec86, Soo87]. We see it roughly shows the same slope tendency of the resonance curve.

\subsection{Cascade scattering by acoustic phonons above the $\left(e, A^{0}\right)$ threshold}

In the experiments, also multi-phonon scattering was observed. It was verified that acoustic phonons participate in the scattering which takes place above the threshold of $\left(e, A^{0}\right)$. This section makes an effort to explain the phenomenon.

The spectra were recorded in the configuration of $Z\left(X^{\prime} X^{\prime}\right) \bar{Z}$. It was found that when the excitation energy was bigger than $1.4953 \mathrm{eV}$, besides the 1-LO Stokes shifted line, another peak with a larger Stokes shift appeared. A typical RRS spectrum in terms of the Stokes shift is shown in Fig. 6.11 (in units of meV), where the "splitted" sharp lines are conspiciously seen. The highest and broadest peak, ascribed to 1-LO phonon replica of impurity luminescence, is not of interest in this section. The zero-phonon luminescence of $\left(e, A^{0}\right)$ and $\left(D^{0}, A^{0}\right)$ is not present within the figure's energy range.

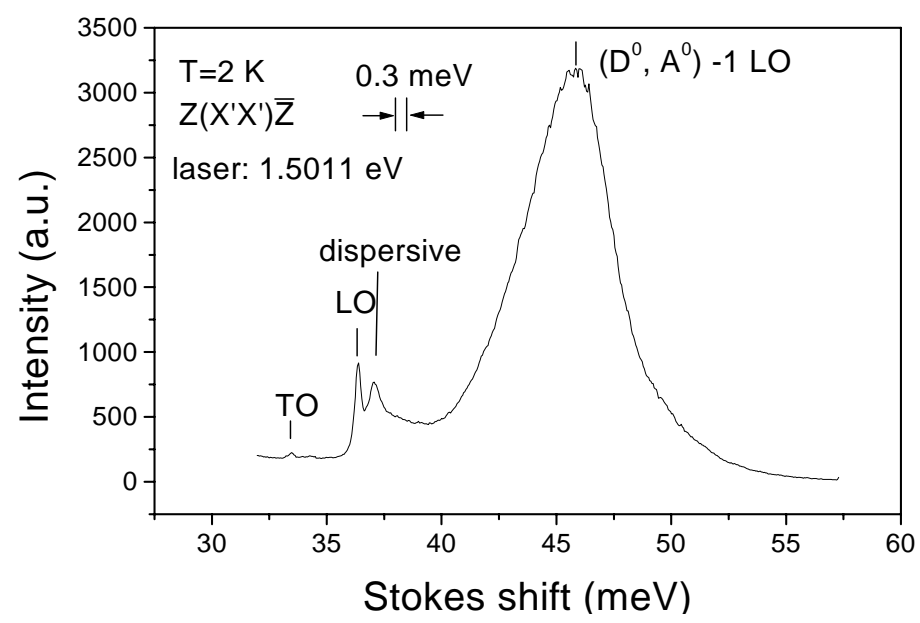

Figure 6.11: A typical RRS spectrum plotted versus "Stokes shift" with excitation energy above the $\left(e, A^{0}\right)$ the threshold. Besides the Raman LO line, there appears another broader line showing a dispersive behavior. The strongest peak is ascribed to 1-LO phonon replica of mixed luminescence of $\left(D^{0}, A^{0}\right)$ and $\left(e, A^{0}\right)$.

This new co-existing line shows a dispersive behavior: its Stokes shift changed with the excitation energy. The measured dispersion relation is shown by the points in Fig. 6.12.

Fig. 6.12 also depicts the the analysis curves based on a calculation which will be explained in the following. Because the normal LO scattering peak is has no significant dispersion (LO phonon frequency is little dependent on phonon wave number $q$ ), splittings 
of the dispersive line relative to this LO line were plotted as a function of excitation energy. The measured splitting increases monotonously with the excitation energy. This dispersion behavior results from the participation of acoustic phonons because the acoustic frequency changes dramatically in the BZ, as introduced in Chapter 2. The dispersion relations for the acoustic phonons, assumed to isotropic and centered at the BZ, are given by $E_{L A}=\hbar v_{L A} q$ and $E_{T A}=\hbar v_{T A} q$. Here we take $v_{L A}=5.5 \times 10^{3} \mathrm{~m} / \mathrm{s}$, and $v_{L A}=3.5 \times 10^{3} \mathrm{~m} / \mathrm{s}$ Bla82].

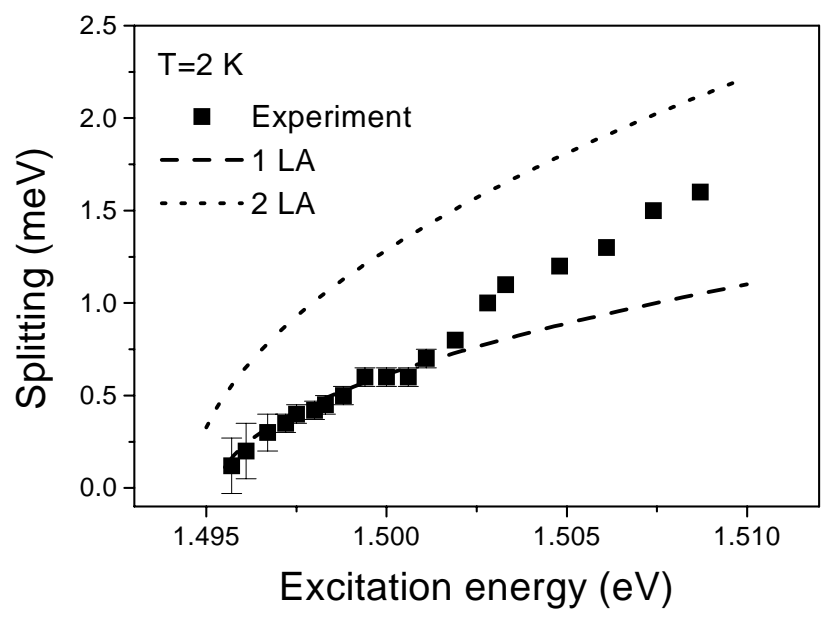

Figure 6.12: Raman energy shift for the dispersive line. The dashed curve is calculated according to Eq. 6.5 which is due to the (1-LO + 1-LA)-scattering process. The dotted curve is based on Eq. $6.4(\mathrm{n}=2)$ for the case of $(1-\mathrm{LO}+2-\mathrm{LA})$-scattering.

To explain the dispersion problem quantitatively, some facts and conditions are highlighted below:

1. The additional scattering sets in at the threshold of $\left(e, A^{0}\right)$.

2. The scattering by LA phonons is stronger than the scattering by TA phonons. A proof can be given: the scattering by LA phonons due to the deformation potential is determined by Eq. 2.7. On the other hand, the piezoelectric electron-phonon interaction responsible for TA scattering is described by Eq. 2.8. So take a rough estimate: if we assume $H_{P E}(\mathrm{TA})>H_{D P-V A}(\mathrm{LA})$, according to the introduction in Chapter 2 we have $q<e \mathbf{e}_{\mathbf{m}} / \epsilon_{\infty} d_{V A}$. Taking the parameter values in the book Yu99: $\left|\mathbf{e}_{\mathbf{m}}\right| \sim 4.8 \times 10^{4} \mathrm{statvol} / \mathrm{cm}$ and $\left|d_{V A}\right| \sim 8.6 \mathrm{eV}$ for GaAs, it follows that the $q$ should be smaller than $5 \times 10^{2} \mathrm{~cm}^{-1}$. However, the $\left(e, A^{0}\right)$ transition is a localized transition process involved with a large magnitude of electron wave vector $k\left(k \sim 10^{5}-10^{6} \mathrm{~cm}^{-1}\right)$. Accordingly it relates to a large $q$-relaxation. Therefore, for large $q$ phonon, the assumption of TA dominance thus does not holds. This fact has also been proved in multi-phonon scattering in other cases with the same reasoning Yu75. The scattering by 1 LA phonon is schematically shown in Fig. 6.13. 
3. An electron in the parabolic conduction band can be scattered successively by many acoustic phonons. But at each step, the scattered LA phonon is assumed to be relaxed with a maximum $q$ via impurity-electron (hole) interaction. It is a logical consequence of point 2 since the rate of LA scattering is proportional to $q^{2}$ Yu75]. The scattering by 2 LA phonons and n LA phonons are schematically exhibited in Fig. 6.14 and 6.15, respectively.

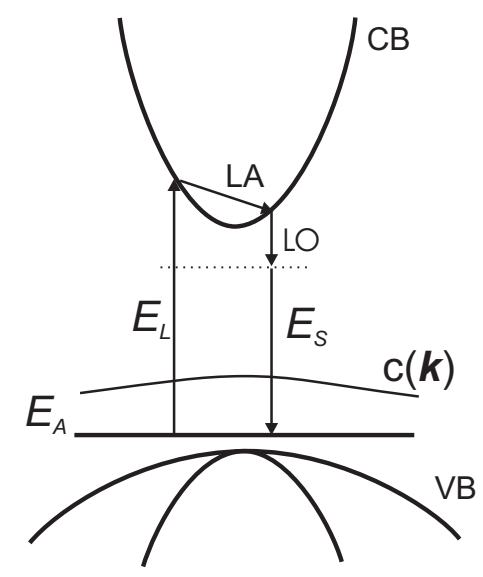

Figure 6.13: The model of multi-phonon scattering by $1 \mathrm{LA}$ phonon is plotted to show that the impurity-induced scattering is a localized process. The $c(\boldsymbol{k})$ is the expansion cofficient of electron's wave vector in the $\boldsymbol{k}$-space.

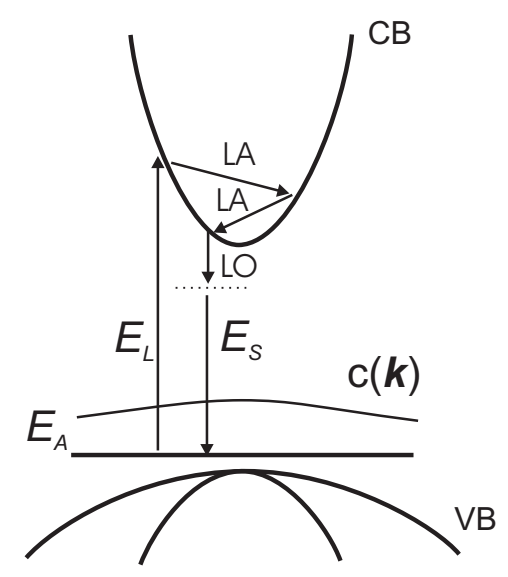

Figure 6.14: The model of multi-phonon scattering by 2 LA phonons.

According to fact 1, we can determine the value of $k$ of an excited electron, which lies 


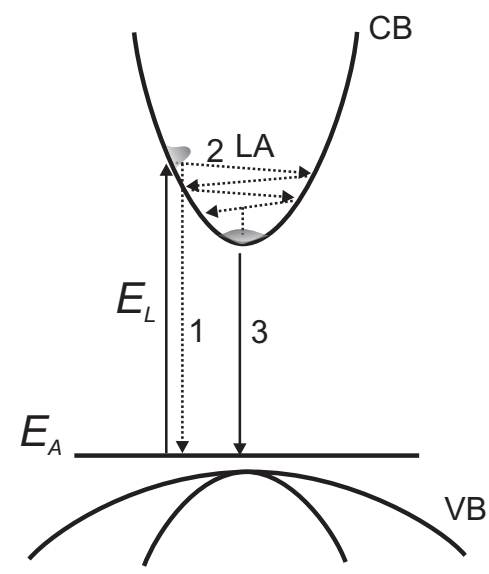

Figure 6.15: The model of multi-phonon scattering by n LA phonons. Notice that the rate of $\left(e, A^{0}\right)$ recombination (process 1$)$ has the order of $\mu$ s if the acceptor concentration is of the order $10^{15} \mathrm{~cm}^{-3}$. The scattering by multiple LA phonons (process 2) may be faster than process 1 , so multi-phonon scattering by LA phonons is quite possible. Many excited electrons may have been relaxed by this scattering mechanism to the bottom of the conduction band, giving out the luminescence (process 3 ).

in the conduction band, by solving

$$
E_{l}=\left(E_{0}-E_{A}\right)+\frac{\hbar^{2} k^{2}}{2 m_{e}}
$$

where $E_{l}$ is laser energy, $m_{e}$ the effective electron mass in the conduction band. In our case, $E_{0}-E_{A}=1.4944 \mathrm{eV}$. For the excitation $E_{l}$ which changes from 1.495 to $1.51 \mathrm{eV}, k$ varies correspondingly from $3 \times 10^{5}$ to $2 \times 10^{6} \mathrm{~cm}^{-1}$.

Based on the point 3, i.e., the maximum $\boldsymbol{q}$-relaxation approximation, the shift (or splitting) with respect to the non-dispersive LO peak is calculated as (for scattering by $\mathrm{n}$ LA phonons)

$$
E_{\text {max }}(n L A)=n\left[2 v_{L A} \sqrt{2 m_{e}\left(E_{l}-E_{0}+E_{A}\right)}-2 m_{e} v_{L A}^{2}\right]-2 n(n-1) m_{e} v_{L A}^{2} .
$$

For 1 LA phonon scattering, from Eq. 6.4 we obtain

$$
E_{\text {max }}(1 L A)=\left[2 v_{L A} \sqrt{2 m_{e}\left(E_{l}-E_{0}+E_{A}\right)}-2 m_{e} v_{L A}^{2}\right] .
$$

The dashed line calculated according to Eq. 6.5 and the dotted line according to Eq. 6.4 with $n=2$ have been shown in Fig. 6.12. For a best fit, $E_{0}-E_{A}=1.495 \mathrm{eV}$ has been taken. With 1-LA-simulation, the fitting is quite satisfactory except when the excitation energy is above $1.502 \mathrm{eV}$. This means that below $1.502 \mathrm{eV}$, the scattering by one LA phonon is dominant. Above $1.502 \mathrm{eV}$, the 1-LA-fitting shows a big discrepancy compared to the experimental points. The simulation with assumption of a pure 2-LA-scattering 
cannot fit the data, either. This implies that there is no a pure 1-LA- or a pure 2-LAscattering when excitation is above $1.502 \mathrm{eV}$. This discrepancy can only be understood when mixed scattering processes are taken into account: when the scattering by 2 LA phonons becomes comparable to the scattering by 1 LA phonon, an overlap of multiple scattering lines, rather than one scattering line, contributes to the spectral peak position. Therefore, the overlapped line will show a larger Stokes shift in the mixed process than in the pure 1-LA-case, but smaller than in the pure 2-LA-case. Proof of this interpretation can also be found in the linewidth analysis, as shown in Fig. 6.16, where the dipersive peak's FWHM is plotted as a function of exciting energy. The estimated FWHM is obtained from an assumed Lorenzian fit. Clearly, the FWHM has no pronounced change until $E_{l}=1.502$ $\mathrm{eV}$. Beyond $1.502 \mathrm{eV}$, it increases considerably with the excitation energy. Of course, when more LA phonons involve in the scattering, the linewidth will be broadened. So it just indicates that a mixed scattering takes place.

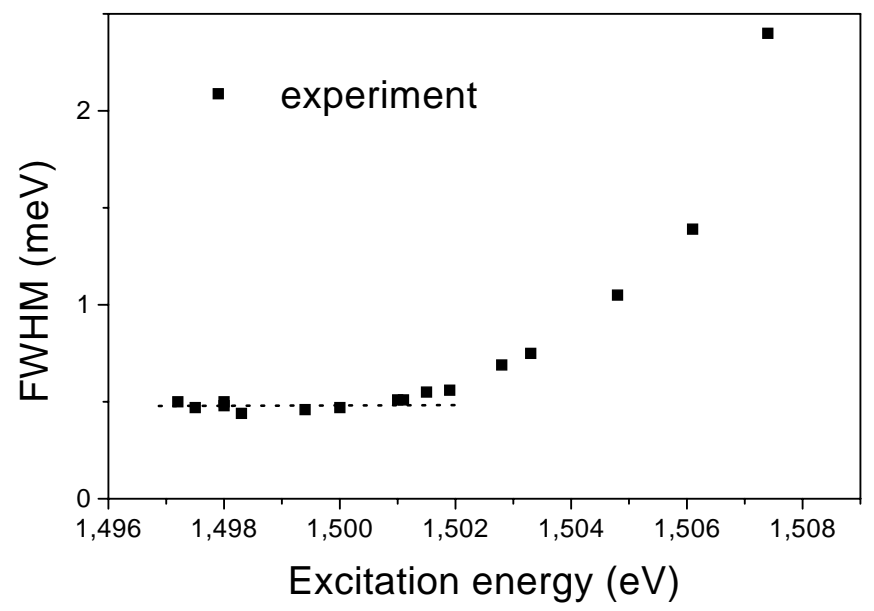

Figure 6.16: The dependence of the linewidth of the dispersive scattering line on the exciting energy. A dashed line is drawn as a visual guide of the measured data. There is no pronounced change of linewith when the excitation is below $1.502 \mathrm{eV}$. Above $1.502 \mathrm{eV}$, the linewidth increases considerably: an indication of mixed n-LA-scattering processes.

To simulate the correct line shape of the scattering spectra, it is necessary to consider the intensity of the scattering. As discussed above, the scattering rate of an electron in a parabolic and nondegenerate band is $\boldsymbol{q}$-dependent. When such an electron emits one LA phonon of $q$ under the deformation potential mechanism, the rate is Yu75

$$
R_{L A}(q, k) \propto \int_{0}^{q} q^{\prime 3}\left(1 / q^{\prime}\right) d q^{\prime} \int \delta\left\{\left[\hbar^{2} q^{\prime} /\left(2 m_{e}\right)\right]\left(k \cos \Theta+q^{\prime}\right)\right\} d(\cos \Theta) \approx q^{2} / k \quad,
$$

where $\Theta$ is the angle between $\boldsymbol{k}$ and $\boldsymbol{q}^{\prime}$. The integration is done over a range of wave vectors which is allowed in the acoustical scattering. For $\boldsymbol{q}^{\prime}$ choice, we plot the LA scattering in the 2-dimentional $\boldsymbol{k}$ space schematically, see Fig. 6.17. 


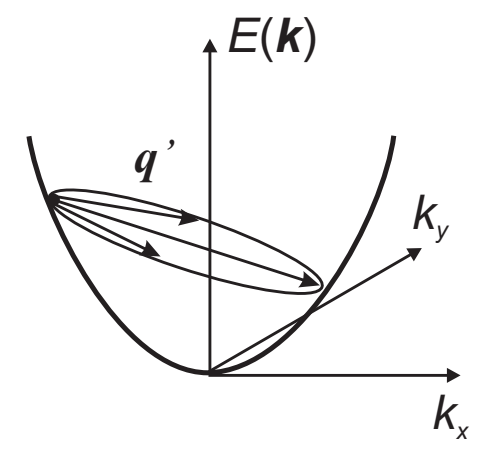

Figure 6.17: The acoustical scattering of an electron in the conduction band. For the intraband acoustic scattering, the final electronic state must still belong to the same band. Thus, the scattered acoustic phonon has a choice of range of wave vectors which are allowed in the scattering, as depicted by the ellipse in the figure. The scattering by a LA phonon with the maximal $q$ has the biggest scattering rate.

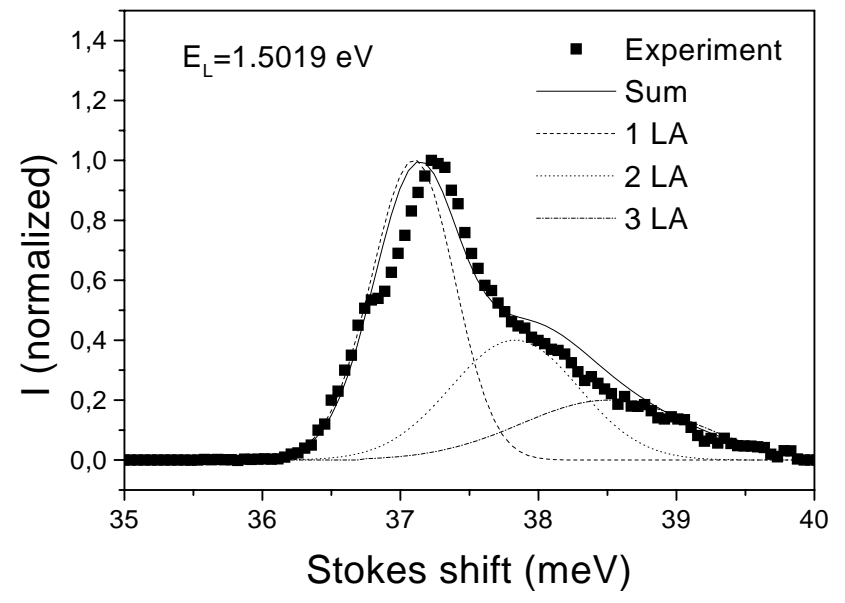

Figure 6.18: An example of simulation of the line shape of the dispersive line with exciting energy $1.5019 \mathrm{eV}$. The dashed curve is only for the pure (1-LO+1-LA)-scattering process, the dotted curve is for the pure $(1-\mathrm{LO}+2$-LA)-process, and the dot-dashed curve is for $(1-\mathrm{LO}+$ 3 -LA)-process. The solid curve is the combination of the three processes, thus the sum of all the contributions. We chose the intensity ratios as $I_{1 L A}: I_{2 L A}: I_{3 L A}=1: 0.4:(0.4)^{2}$ in the simulation.

In analogy with the fomulation of $\mathrm{Yu}$ and Shen for $\mathrm{Cu}_{2} \mathrm{O}$ [Yu75], by substituting Eq. 6.3 into Eq. 6.6 for the one LA phonon scattering, one obtains a qualitative formula to estimate the line shape for the scattering:

$$
I_{1 L A}\left(\delta=\omega_{l}-\omega_{s}\right) \propto \int_{0}^{\omega_{\max }} d \omega^{\prime}\left(\omega^{\prime}\right)^{2} \exp \left\{-\left[\left(\omega^{\prime}-\delta \omega\right) / \Delta\right]^{2}\right\} \quad .
$$


Here a Gaussian spectrometer transmission function is convoluted with $\Delta \approx 3 \mathrm{~cm}^{-1}$. $\omega_{\text {max }}=2 v_{L A}\left(k-m_{e} v / \hbar\right)$, where $k$ is determined by Eq. 6.3. The dotted line is obtained according to this evaluation.

For scattering involved with two or more LA phonons, the evaluation can be obtained in a similar way. But the scattering rate for the higher order processes decrease dramatically with the increase of the phonon number involved. As an example, the overlapped line due to three combined scattering processes is simulated for the case $E_{L}=1.5019 \mathrm{eV}$. Fig. 6.18 qualitatively illustrates the overlapping by setting a variable intensity ratio in simulation. It is seen in Fig. 6.18 that this analysis can explain not only the peak position and the linewidth, but also the line's asymmetric line shape. With overlapping consideration, the "tail", which is tended to the larger shift side, is thus simulated in good agreement with the experimental line's profile.

As mentioned in Chapter 1, the multi-phonon RRS by LA phonons has been observed only via discrete exciton states in semiconductors. In the exciton case, the scattering starts with an e-h pair in an intermediate state with $K=0$, then the e-h pair is excited resonantly into an exciton-polariton state assisted by emitting a LA phonon. After scattered by additional LO phonons, the recombination happens, giving out the Raman scattering light. Such a LA-Phonon-assisted absorption in the scattering is denied in our case, because the $\left(e, A^{0}\right)$ transition is localized at acceptors thus involves large $k$. We also have tried to fit the Stokes shift of the dispersive line by assuming that the transition of $\left(e, A^{0}\right)$ is non-localized with $k=0$, see the scattering model in Fig. 6.19. No convincing and consistent agreement with the experimental data can be obtained in the simulation. Therefore, we have found a new kind of LA-phonon-involved Raman scattering mediated by free-to-bound transition. It is non-discrete-exciton mediated and it is the scattering by one or more acoustic phonons before the e-h recombination happens.

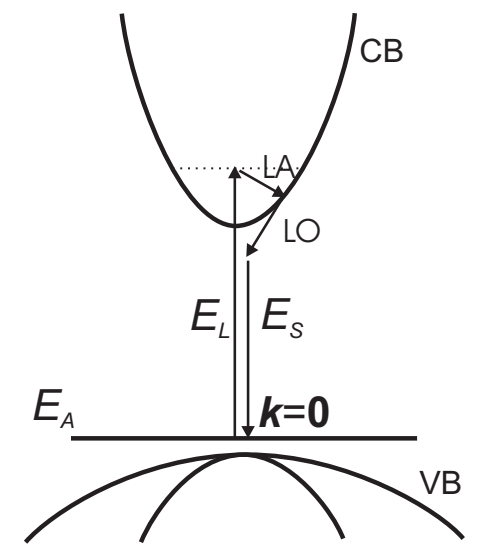

Figure 6.19: An assumed model for the multi-phonon scattering at $\left(e, A^{0}\right)$, where the excitation happens at $k=0$, then scattered by a LA phonon into the conduction band, followed by the LO scattering and the recombination.

To summarize this section, we have analyzed that impurity-induced scattering involves 
acoustic phonons when the excitation energy is above the $\left(e, A^{0}\right)$ threshold. Thus we have found that LA-mediated scattering dominates the relaxation of photo-excited hot electrons.

\subsection{First-order RRS at $\left(A^{0}, X\right)$}

This section briefly discusses Raman scattering with resonance at $\left(A^{0}, X\right)$. The RRS at bound exciton has been studied in CdS by Damen, Colwell et al. before [Col70, Dam71]. Although the impurity concentration was low, they found that the scattering efficient was fairly high. They explained the scattering enhancement by considering the "giant" oscillator strength of the bound exciton. Here we analyze the RRS at $\left(A^{0}, X\right)$ in GaAs in a similar way.

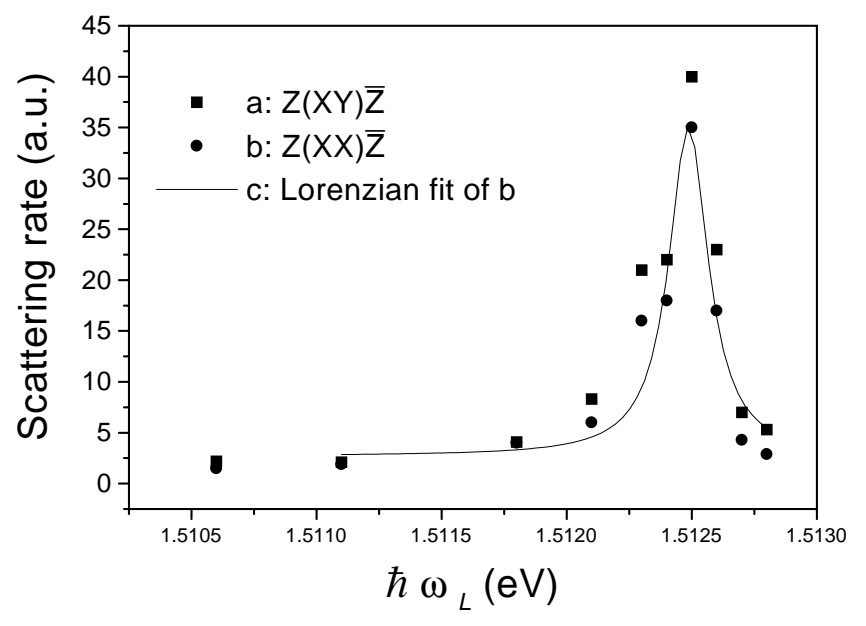

Figure 6.20: Resonance Raman scattering at $\left(A^{0}, X\right)$. The presented data are scattering rate recorded in the experiment directly, without the correction of light absorption, transmission and etc., as done in the $\left(e, A^{0}\right)$ case. A Lorentzian fit of the measured points in the $Z(X X) \bar{Z}$ configuration is tried so as to explain the exciton's F-induced scattering.

Fig. 6.20 shows a sharp resonance at $1.5125 \mathrm{eV}$, which corresponds exactly to the impurity bound exciton transition: $\left(A^{0}, X\right)$. The scattering intensities have been measured in the different configurations, however, no polarization selection rules have been essentially obeyed. As assumed that the F-induced scattering plays a dominant role in the configuration $Z(X X) \bar{Z}$ [Col70], we find that a Loranzian fit can simulate the data. From the fitting, a damping constant for the bound-exciton with value of $0.2 \mathrm{meV}$ is obtained. The explanation of such a fit is given below.

For the RRS at $\left(A^{0}, X\right)$, where the intermediate state is a bound exciton, an analogous analysis in Raman polarizability can be carried out by referring to the free exciton based RRS theory. Two correlations, however, should be made:

- the matrix element of $H_{E R}$ for bound exciton instead of free exciton; 
- the matrix element of $H_{E P}$ between bound exciton and phonon instead of between free exciton and phonon.

The intermediate state is the acceptor-bound exciton given by Eq. 5.29. The centerof-mass wave function in the acceptor-localized weak potential $\Phi_{B X}(\boldsymbol{R})$ is given by Eq. 5.38. For the first correlation, the intensity of e-h recombination in the scattering can be estimated by the oscillator strength in Eq. 5.31, which shows the enormous enhancement compared to the oscillator strength of free exciton: $f_{B X} / f_{X} \sim 10^{5}$. For the second correlation, the intermediate state is now a bound exciton, so the matrix element due to the Fröhlich interaction should be revised.

The Fröhlich interaction is given by Eq. 2.10. We re-write it here as

$$
H_{F}(q, r)=\sum_{q}\left(i C_{F} / \sqrt{N v_{0}} q\right)\left\{c_{q}\left(e^{i q r_{e}}-e^{i q r_{h}}\right)-c . c .\right\}
$$

The matrix element between two 1-s free exciton states is

$$
<X\left|H_{F}(q, r)\right| X>=i C_{F} F(q) / q\left(c_{-q}^{*}-c_{q}\right)
$$

where $F(q)$ is given by

$$
F(q)=\int \phi(r)^{2}\left(e^{-i q r m_{e} /\left(m_{e}+m_{h}\right)}-e^{-i q r m_{h} /\left(m_{e}+m_{h}\right)}\right) d^{3} r
$$

with $\phi(r)$ the envelop wave function of the free exciton:

$$
\phi(r)=\sqrt{\frac{1}{\pi a_{X}^{3}}} e^{-r / a_{X}} .
$$

The matrix element between two bound exciton states thus is modified as

$$
<B X\left|H_{F}(q, r)\right| B X>=i C_{F} F(q) / q\left(c_{-q}^{*}-c_{q}\right) G(q)
$$

where $G(q)$ is given by

$$
G(q)=\int \Phi_{B X}(R)^{2} e^{i q R} d^{3} R
$$

with $\Phi_{B X}$ given by Eq. 5.32 or Eq. 5.38 .

As a rough estimate, $G(q) \sim \pi / q R_{e} \sim a_{X} / R_{e} \sim 1$ Col70], where $R_{e}$ denotes the radius of the mass center movement. So this modification would not result in much change for the scattering intensity if taking the bound exciton effect into account.

With the above two correction, we can estimate the absolute value of Raman intensity of bound exciton $\left(A^{0}, X\right)$ scattering. Obviously, the total Raman intensity of the crystal is a function of oscillator strength and impurity density:

$$
a^{2} \propto f^{2} N_{A} \Sigma
$$


where $\Sigma$ is the quantity summing over all the possible scattering with different $q$

$$
\Sigma=\sum_{q}|<q| H_{F}|0>|^{2}
$$

Neglecting the possible scattering enhancement due to the $q$-relaxation effect, we find that the "giant" oscillator strength effect can yield the net gain of several orders in magnitude alone compared to the scattering of free excitons:

$$
\frac{a_{B X}^{2}}{a_{X}^{2}} \sim \frac{f_{B X}^{2}}{f_{X}^{2}} N_{A} v_{0} \sim 10^{3}
$$

Although we did not get the reliable absorption coefficient for the $\left(A^{0}, X\right)$ because the transmission light through the bulk sample $(450 \mu \mathrm{m}$ in thickness $)$ in the bound exciton absorption energy region near $1.51 \mathrm{eV}$ is too weak to be detected, we have identified the RRS at the $\left(A^{0}, X\right)$ evidently in the experiment, and the enhancement order given by Eq. 6.16 is in agreement with our rough evaluation of the measured intensity. The data in Fig. 6.20 are just the measured intensity outside the crystal without the correction, but they can be fitted by the Lorentzian function well because of this bound-exciton is similar the RRS of free exciton, to which the theory introduced in the section 2.5 .2 can be applied (i.e., the dominant discrete-to-discrete term). 


\section{Chapter 7}

\section{Other spectral features due to impurity spatial distribution effect}

This chapter discusses some spectral features due to many-particle effects. The spatial distribution of donors and acceptors in the samples is taken into account.

When the laser excites impurity transitions resonantly, both laser stray light and impurity luminescence are emitted. The laser stray light is only due to the roughness of the sample surface thus has little usage in the spectral analysis. However, the stray light may be so strong that it creates difficulty to record the so-called resonant impurity luminescence, i.e., the impurity luminescence emitted near the laser exciting energy. A method to circumvent this difficulty is to record and analyze the luminescence phonon replicas instead the luminescence itself. This method can investigate resonant impurity luminescence efficiently owing to two reasons:

- the laser stray light can be rejected efficiently by the spectrometer when recorded with a relatively large frequency shift, i.e., the phonon side-band;

- the replica signals just reflect those impurity transitions, keeping the similar spectral shapes of the direct PL signal in the regime of excitation energies. See Fig. 4.1. where the 1-LO-replica and 1-LO-replica remain the same profile as their original luminescence.

Therefore, in the following we will discuss spectra of luminescence's phonon replica instead of resonant impurity luminescence itself, while keeping in mind that the discussion relates to the impurity transitions directly.

So this chapter begins with the discussion of the phonon replicas of luminescence. Then, the spectral properties due to impurity spatial distribution are explained thereafter.

\subsection{Phonon replicas of luminescence}

The impurities inside the crystal can induce lattice distortions. Therefore the recombinations at the impurities are often accompanied by absorption or generation of phonons. 
Reflected in the PL spectra, the phonon replicas of luminescence can be observed.

This phenomenon, which is actually due to the coupling between phonons and electrons in the localized transitions, can be described conveniently on the basis of the configurationnormal-coordinate model, as treated in the references [Mal71, Toy70]. The configuration coordination represents the movement of the nuclei in the lattice. The movements of the nuclei are dependent on each other and can be identified as many different and uncoupled normal-vibration-modes. In the configuration-normal-coordinate model, the normal coordinates are simply introduced to describe the collective movement of the nuclei.

Because this model has taken the coupling between electron and phonon into account directly, the appearance of phonon replica in the electronic transitions can be understood in an intuitive way, as depicted schematically in Fig. 7.1. Notice that the equilibrium position of the excited state $Q_{0}$ has a shift $\Delta$ towards the outside with respective to that of ground state. This is just an impurity-induced lattice distortion effect. The electron are coupled by LO phonons, mainly due to Fröhlich mechanism.

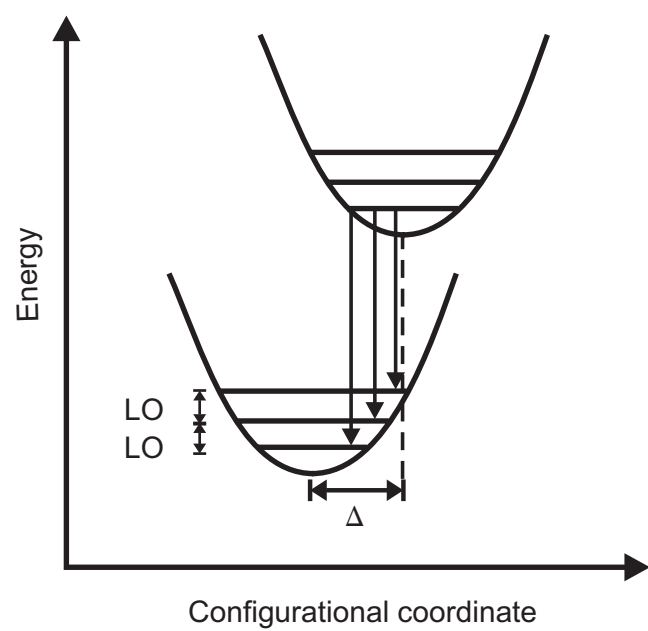

Figure 7.1: Adiabatic potentials in a one-dimensional configurational coordinate model for localized transitions. Vertical arrows represent LO-phonon-assisted emissions.

In the adiabatic approximation, the combined electron-lattice wave function $\Psi_{i \alpha}(r, Q)$ may be written as

$$
\Psi_{i \alpha}(r, Q)=\phi_{i}(r, Q) \chi_{i \alpha}(Q),
$$

where $\phi_{i}(r, Q)$ refers to the electronic part of the wave function and $\chi_{i \alpha}(Q)$ refers to the vibrational part of the lattice. Here $r$ denotes electron coordinator, and $Q$ the normal coordinator.

The total Hamiltonian $H(r, Q)$ can be expressed with three distinct parts:

$$
H(r, Q)=H_{E}(r)+H_{P}(Q)+H_{E P}(r, Q),
$$

where $H_{E}, H_{P}$ and $H_{E P}$ represent the Hamiltonians of the electrons, phonons and the electron-phonon interaction, respectively. 
The Schrödinger's equation then separates as follows [Mal71]:

$$
\left[H_{E}(r)+H_{E P}(r, Q)\right] \phi_{i}(r, Q)=E_{i}(Q) \phi_{i}(r, Q)
$$

and

$$
\left[H_{P}(Q)+E_{i}(R)\right] \chi_{i \alpha}(R)=E_{i \alpha}(R) \chi_{i \alpha}(R) \quad .
$$

If harmonic vibration is assumed, the ground state $E_{g}=\frac{1}{2} M \omega_{0}^{2} Q^{2}$. The excited state $E_{e}=\frac{1}{2} \omega_{0}^{2}\left(Q-Q_{0}\right)^{2}+E_{e}^{0}$, where $Q$ is normal mode coordinator, $\omega_{0}$ is vibrational frequency,

The number of phonons $S$ emitted just after the absorption transition at $Q=0$ is

$$
S=\frac{1}{2} \omega_{0}^{2} Q_{0}^{2} / \hbar \omega_{0}=\omega_{0} \Delta^{2} / 2 \hbar
$$

$S$ is regarded as a replica scattering coefficient expressing the strength of the electronlattice interaction.

The transition probability is proportional to the square of the dipole matrix element: $P=\left|\left\langle\Psi_{e j}|r| \Psi_{g i}\right\rangle\right|^{2}$. Using the Frank-Condon assumption, which states that the dipole moment is independent of $Q$, we can separate $P$ as $\left|\left\langle\phi_{e}|r| \phi_{g}\right\rangle\right|^{2}\left|\left\langle\chi_{e j} \mid \chi_{g i}\right\rangle\right|^{2}$. Thus, the spectra of replicas are determined by calculating the overlap integral of $\left|\left\langle\chi_{e j} \mid \chi_{g i}\right\rangle\right|^{2}$. When emission from the state $j=0$ to states $i$ happens as shown in Fig. 7.1, the emission probability $P_{0 i}$ is proved to has a Poisson distribution:

$$
P_{0 i}=e^{-S} \frac{S^{i}}{i !}
$$

To handle the problem of LO-phonon-replicas of luminescence $\left(e, A^{0}\right)$, one needs to know both the lattice-phonon interaction and the the electron (hole) wave functions in ground and excited state. The interaction $H_{E P}$ may be expanded in terms of the normal coodinators $Q_{i}$. Keeping the linear term only, one has

$$
H_{E P}=\sum_{l} T_{l}(r) Q_{i}
$$

with $T_{l}(r)$ the expansion coefficient.

Thus the equilibrium position shift can be calculated as

$$
\Delta=\frac{1}{\omega_{0}^{2}} \int\left[\left|\phi_{g}\right|^{2}-\left|\phi_{e}\right|^{2}\right] T_{l}(r) d^{3} r
$$

For the case $\left(e, A^{0}\right)$, the LO replicas are mainly due to Fröhlich interaction, so the coefficients $T_{l}$ in Eqs. 7.7 and 7.8 is taken as

$$
T_{l}=\left[\frac{4 \pi \omega_{0}^{2} e^{2}}{N v_{0}}\left(\frac{1}{\epsilon_{\infty}}-\frac{1}{\epsilon_{0}}\right)\right]^{1 / 2} \frac{1}{k} e^{i k r} .
$$

The excited $\left(e, A^{0}\right)$ state is just at acceptor level with an approximated hydrogenic wave function given by Eq. 5.19. We rewrite the envelope function as 


$$
\phi_{e}=\left(\frac{1}{\pi a_{A}^{3}}\right)^{1 / 2} \exp \left(-\frac{r}{a_{A}}\right),
$$

Inserting Eqs. 7.9 and 7.10 into Eq. 7.8, one thus can figure out Eq. 7.5 with a good approximation:

$$
S \simeq \frac{e^{2}}{\hbar \omega_{0}}\left(\frac{1}{\epsilon_{\infty}}-\frac{1}{\epsilon_{0}}\right) \frac{5}{16 a_{A}}
$$

For the case $\left(D^{0}, A^{0}\right)$, because donor state has a much more extended wave function than acceptor state, the localization is mainly controlled by acceptor state. Therefore, Eq. 7.11 still holds valid to compute the replica intensity from $\left(D^{0}, A^{0}\right)$, too.

Evaluating Eq. 7.11 with the parameter values of GaAs (Appendix A), we have $S \sim$ 0.05. In the experiment, $S / 2=\frac{I_{2 L O}}{I_{1 L O}} \sim 0.024$ was obtained, as shown in Fig. 4.1 in Chapter 3. So it is in agreement with the theoretically estimated value 0.05 .

\subsection{Donor pairs}

The peak around $1.454 \mathrm{eV}$ corresponds to the $\left(D^{0}, A^{0}\right)$ recombination. As analyzed in Chapter 3, if the recombination only occurs for DAP, there should be a lower-limit for the spectrum of $\left(D^{0}, A^{0}\right)$ at low energy side, which corresponds to the pair separation approaching the mean separation distance. However, no such a down-limit can be identified in in the PL spectrum. Instead, there is a "tail" extending to the low energy side, see Fig. 4.1 .

To understand this spectral feature, one should take impurity spatial distribution feature into account. Here we provide an example by considering donor pairs, which may suffice to illustrate some detailed features of the spectra.

Let us consider the system with an electron bound to a close ionized donor pair. If without the appearance a second adjacent ionized donor, i.e., without the perturbation of another charged particle, the electron is bound to the donor with Bohr radius about $100 \AA$. But if the second donor ion appears and being close to the first one with separation less than $100 \AA$, thus forming a donor pair, an electron could be trapped by this closely paired donors.

This case is similar to a $\mathrm{H}_{2}^{-}$ion. See Fig. 7.2. Because the donors have fixed positions in the lattice, the Coulomb potential between the donor pairs need not be included in the Hamiltonian to solve the electron state. The Schrödinger's equation for the electron in the three-particle system is thus simplified as (in atomic units)

$$
\left(-\frac{1}{2} \nabla^{2}-\frac{1}{r_{a}}-\frac{1}{r_{b}}\right) \phi=E_{s} \phi
$$

The ground state for system can be constructed with approximation in the form of

$$
\phi=c_{1} \phi_{a}+c_{2} \phi_{b}
$$




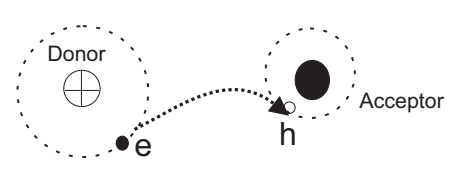

(a)

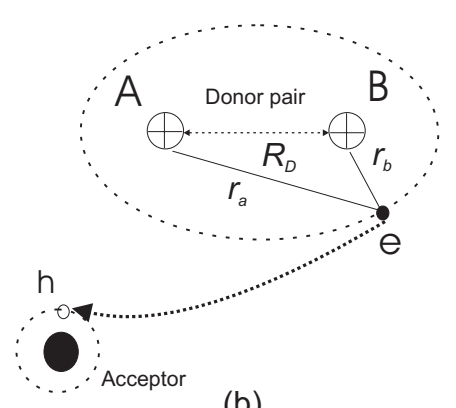

(b)

Figure 7.2: An illustration of donor pair effect on the luminescence spectrum. (a) Recombination in a single donor-acceptor pair, giving out luminescence of $\left(D^{0}, A^{0}\right)$. (b) Due to closed donors (donor pair) in the real space, recombination also exists between an acceptor and a donor pair complex, which emits light with energy below $\left(D^{0}, A^{0}\right)$.

with

$$
\phi_{a(b)}=\frac{1}{\sqrt{\pi r_{D}^{3}}} e^{-r_{a(b)} / a_{D}}
$$

For the bound state, the normalized eigen-state and eigen-energy are

$$
\phi_{s}=\frac{1}{\sqrt{2+2 O}}\left(\phi_{a}+\phi_{b}\right), \quad E_{s}(R)=\frac{H_{a a}+H_{a b}}{1+O},
$$

where

$$
O=\operatorname{Re} \int \phi_{a}\left(r_{a}\right) \phi_{b}\left(r_{b}\right) d^{3} r, \quad H_{a a}=\int \phi_{a}^{*} H \phi_{a} d^{3} r_{a}, \quad H_{a b}=\int \phi_{a}^{*} H \phi_{b} d^{3} r_{b}
$$

Hence, the relation of $R_{D}$ and $E_{s}$ is established by Eq. 7.15. The analytic solution of $\mathrm{H}_{2}^{-}$ ion is found in standard text books of quantum mechanics, for example, in book [Dem96]. Here we just have different parameters like Bohr radius $a_{D}$ and dielectric constant. If the distribution of donor pairs is known, which is a function of $R_{D}$ and corresponds to definite emission energy, the spectrum thus can be simulated.

Approximately, for the randomly distributed donors in a 3-dimensional lattice, the number of configurations for a pair with distance $R_{D}$ is proportional to $R_{D}^{2}$. With the relationship between $R_{D}$ and $E_{s}$ given by Eq. 7.15, we obtain the density of donor pair states versus the luminescence energy. The simulated is shown in Fig. 7.3, where we see clearly that a "tail" extended to the lower side of DAP luminescence $\left(D^{0}, A^{0}\right)$. So the spectral "tail" at lower energy side is simply understood as one of many-particle effects on the spectrum. 


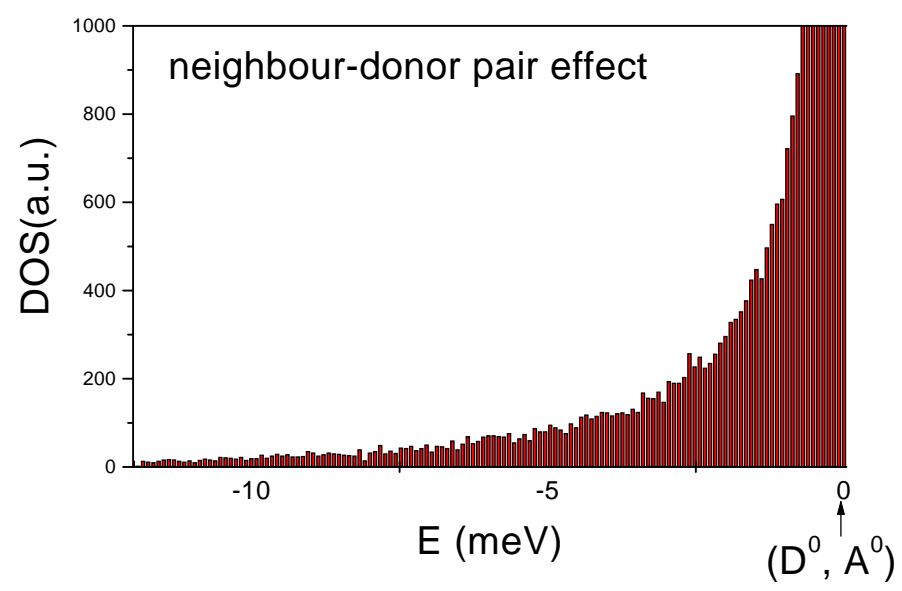

Figure 7.3: Simulation of density of states for donor-pairs: the spectral "tail" below $\left(D^{0}, A^{0}\right)$ is due to donor pair effect.

\subsection{Selective pair luminescence}

It was observed that, when resonantly exciting the impurity transitions, namely, the $\left(D^{0}, A^{0}\right)$ and $\left(e, A^{0}\right)$, the emission happens with a relatively much extended energy in spectrum, both to lower energy side and higher energy side of the excitation energy. See Figs. 6.1, 6.2 and 6.3. The RRS siganl overlaps the replica signal and the replica signal is not symmetrical in the line shape. We explain this phenomenon in terms of "selective pair luminescence" (SPL).

At first we clarify our definition of the term of "selective pair luminescence". In the literature, SPL stands for a spectral method which was developed to identify the excited impurity states under resonant excitation conditions [Ash75, Kis83, Mac89, Tew79a, Tew79b, Wag86. The proposal is based on such a recombination mechanism: first, the incident phonon creates a donor-excited acceptor pair at a particular pair separation; secondly, the excited acceptor is rapidly de-excited to its ground state since the relaxation time of the hole is much shorter than the DAP lifetime; finally, the initially created DAP recombines, emitting a luminescence photon. In such a scheme, the pair is produced at excited acceptor state, and the excitation and recombination occurs within the same DAP. The line shape and lifetime of the pair luminescence due to this mechanism dependent of the DAP spatical distribution have been studied as well [in88, Iin90, Har90].

In our definition, we refer the term selective pair luminescence to the case where emission involes only ground states but among different transition centers in the real space. Because in the experiment high intensity excitation was used in obtaining the PL spectrum, a huge number of DAP were excited simultaneously, with the separation determined by excitation energy, see Eq. 5.42. On the other hand, these DAP are not "isolated" in the space, i.e., in recombination they may lose the memory of their excitation history. This concept has been 


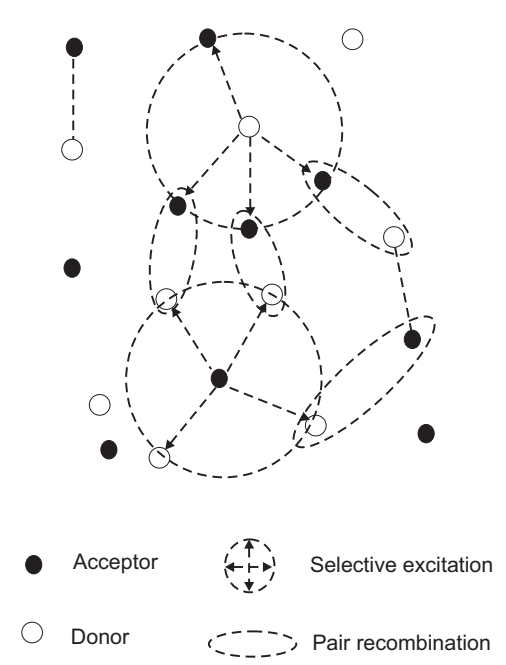

Figure 7.4: A diagram to explain the "selective pair luminescence". Excitation of DAP with a special separation is determined by the laser energy. After the excitation, a special distribution is formed and then the recombination can happen for the nearest DAP with the biggest probability. Therefore, the excitation and recombination may take place amongst different DAP.

schematically demonstrated in Fig. 7.4. So in our case, the influence of the surrounding particles to the recombination of one selectively excited DAP should be taken into account.

We still use the same term as in the literature because the basic meaning of the term holds for both definitions: the DAP are excited selectively in space by laser; the excitation energy determines the separation of the excited DAP.

This so-defined selective pair luminescence has naturally an extended spectrum. In Fig. 7.5, we simulate the situations when laser is tuned to excited differently-separated DAP. In the simulation, impurities are randomly distributed in a two-dimensional lattice. Excitation energy determines the DAP with a corresponding pair distance determined by Eq. 5.42 (the $E_{l u m}$ in Eq. 5.42, however, should be replaced here by excitation energy $E_{s l}$ ), thus creating selectively a definite DAP distribution. Then, these DAP start to recombine, giving out luminescence. However, in the recombination, they lose the memory of excitation history, with the radiative rate only dependent on the wave function overlap between the acceptor and donor in a closed pair. Therefore, the existence of many surrounding particles makes luminescence peak broader. In Fig. 7.5, one can see a "tail" extended to the lower side (left side) of the excitation energy when the laser excites the DAP with a mean separation (600 Åin the simulation) resonantly.

\subsection{Up-converted luminescence}

In the case of the SPL discussed above, the excitation and recombination can take place in different donor acceptor pairs with consideration of impurity spatial distribution. Although 

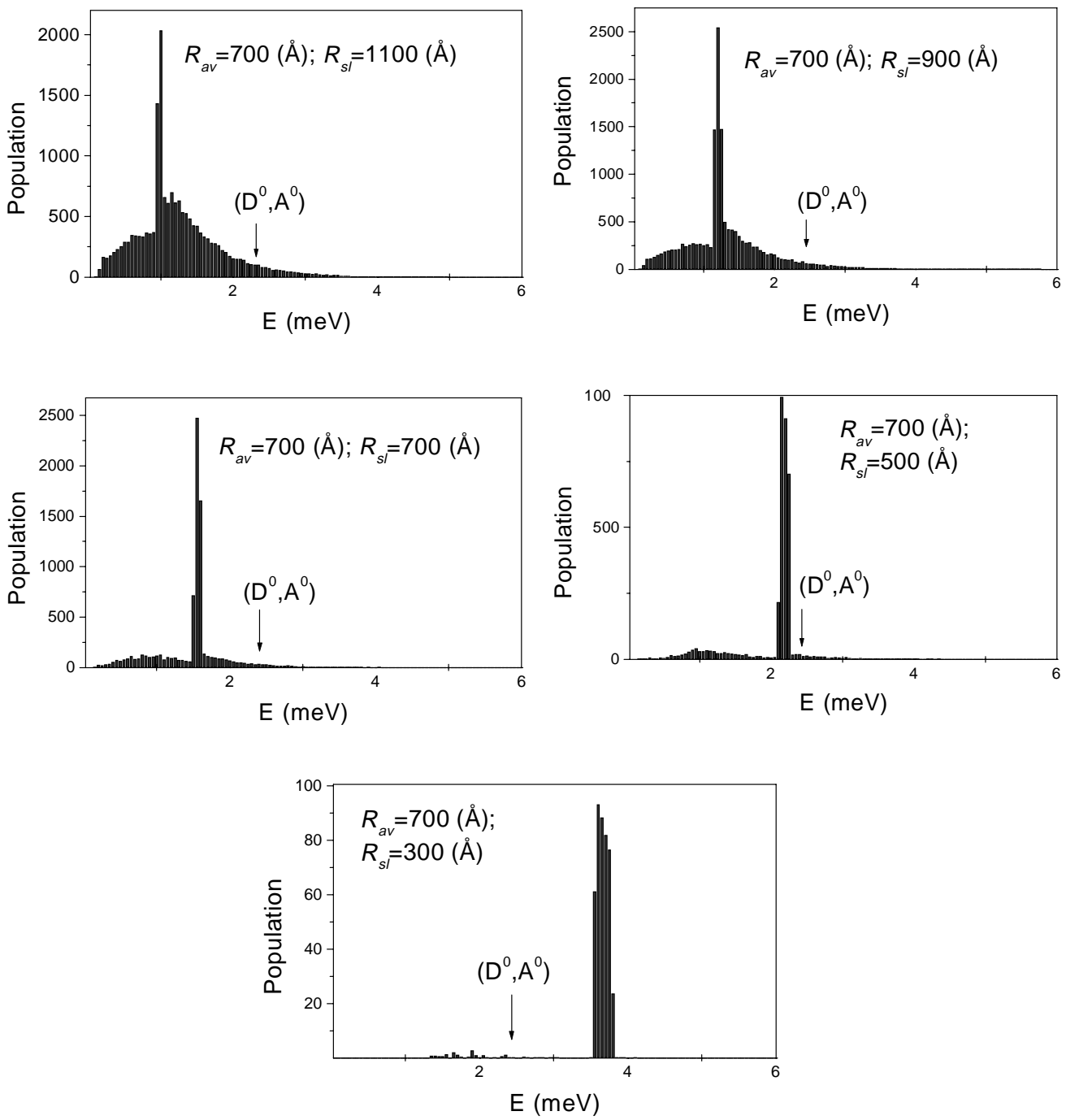

Figure 7.5: Simulation of the closed DAP population contributing to emission band in a 2dimensional lattice. Before the recombination, those DAP are created selectively by excitation energy, accordingly, are selected by separation $R_{s l} . R_{a v}$ is the mean separation of DAP. The $\left(D^{0}, A^{0}\right)$ position in the plots just correspond to DAP with the mean separation $600 \AA$. The peak position corresponds to excitation energy. The spectrum is extented to both sides of the peak.

closer pairs have smaller occurrence in the crystal, the recombination rate is higher. Thus the possibility for up-converted luminescence exists. So there is luminescence extended beyond the excitation energy, too. This luminescence with energy higher (right side) than the excitation energy has actually been observed under the resonant excitation condition, see Fig. 6.2 and simulation results in Fig. 7.5. Because this up-converted luminescence is produced when laser excites the impurity transitions resonantly, it is also called resonant 
impurity luminescence.

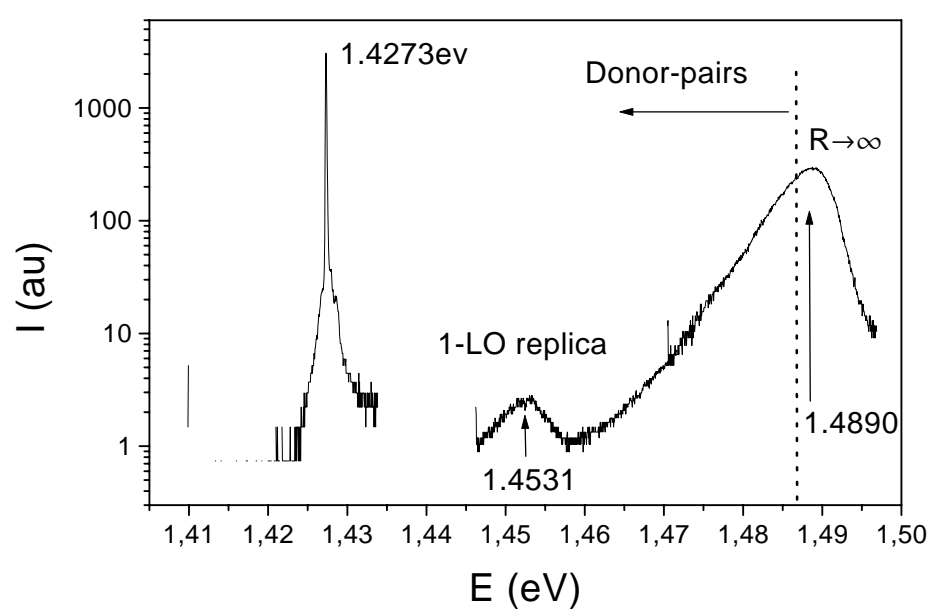

Figure 7.6: Spectrum of up-converted luminescence. The laser at $1.4273 \mathrm{eV}$ is far below the up-converted luminescence peak of $\left(D^{0}, A^{0}\right)$ at $1.4890 \mathrm{eV}$, which corresponds to the DAP with a large separation, or, $R \rightarrow \infty$. This up-converted luminescence is due to two-step absorption via a deep center. Also notice that an long extended "tail" below the up-converted $\left(D^{0}, A^{0}\right)$ peak, which just stems from the emission of donor pairs which has been explained in Section 7.2 .

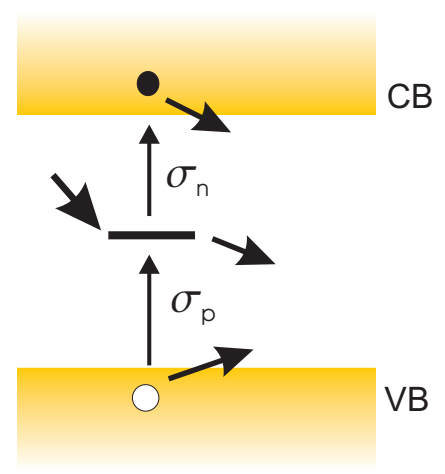

Figure 7.7: Schematic model of two-step absorption via a deep center, which may be the main mechanism for the observed up-converted luminescence.

Here we focus on another kind of phenomenon of up-converted luminescence which has a totally different mechanism. When the GaAs sample was excited by excitation energy much lower than $\left(D^{0}, A^{0}\right)$, thus the DA cannot be resonantly excited, the DAP luminescence was still observed. This up-converted luminescence cannot be ascribed to resonant impurity luminescence and instead, it is called anti-Stokes emission, characterized by a band edge emission with a relatively fixed energy position independent of exciting 
energy and doing little with the spatially selective effect. Fig. 7.6 gives an example of such an up-convered PL spectrum. Here the up-converted luminescence is actually by means of a two-step optical excitation process which creates electron-hole pairs via an intermediate deep center. This up-converted luminescence due to deep center has been studied in Refs. Mor91, Ale97, Joh82, Qua84.

One of possible mechanisms for the two-step process for the electron-hole recombination assisted by an intermediate state is suggested as (Fig. 7.7):

- in step A, an electron is excited from the valence band to the deep center X: $X^{n+}+\hbar \omega \longrightarrow X^{(n-1)+}+h$.

- in step B, the electron transfers from the center $\mathrm{X}$ to the conduction band: $X^{(n-1)+}+\hbar \omega \longrightarrow X^{n+}+e$;

In such a two-step process, electrons and holes are thus generated respectively in the conduction and valence band, whereas the deep center remains unchanged.

The deep center could be a donor or an acceptor due to doping effect. However, for semi-isolating undoped GaAs, it has been proposed that EL2 (antisite defect) Kau89] can be an most attractive candidate as the deep center. It is located at $0.75 \mathrm{eV}$ above the valence band. It could be responsible for the up-conversion phenomenon in our experiment.

The intensity dependence of the up-converted luminescence on the excitation power shows a more or less linear dependence, which is a suggestion that saturation of one of the two transitions takes place.

In conclusion, in this chapter we have accounted for the detailed spectral features of RRS signal which is influenced by impurity-related luminescence by considering the impurity spatial effect and other factors responsible for the observed luminescence. 


\section{Chapter 8}

\section{Summary}

This thesis has focused on the interpretation of the impurity-induced resonance Raman scattering below the $E_{0}$ gap at low temperature in GaAs. Spectral analysis related to the phenomena of the impurity-induced RRS has been carried out in detail.

Central results of the research are highlighted in the following:

1. The first-order impurity-induced RRS below the $E_{0}$ gap has been investigated in GaAs at $2 \mathrm{~K}$. The resonance at the threshold of $\left(e, A^{0}\right)$ has been confirmed and analyzed. Intraband Fröhlich interaction is one of the main mechanisms for the impurity-induced LO scattering. It is found that the impurity-induced scattering is quite strong compared to the intrinsic scattering. The large scattering cross-sections can be understood by considering the large oscillator strength of the $\left(e, A^{0}\right)$ transition. In the impurity-induced scattering, the selection rules are broken due to the $\boldsymbol{q}$-relaxation effect. We have also attempted to explain the scattering intensity quantitatively by referring to RRS theory for uncorrelated e-h pairs with the assumption of "acceptor-valence-band" and have found the fitting above the $\left(e, A^{0}\right)$ threshold is in agreement of the experimental data. However, we have also found that theory based on the uncorrelated pairs cannot explain the sharpness of the resonance below the $\left(e, A^{0}\right)$ threshold. So we think that the exciton effect, i.e., inclusion of Coulomb interaction between the e-h pairs, may explain the rapid increase of scattering intensity near resonance.

2. Multi-phonon scattering has been studied, which takes place above the $\left(e, A^{0}\right)$ threshold. The dispersive scattering line is ascribed to the scattering by LA phonons. The electrons are scattered by acoustic phonons before successively being scattered by one LO phonon and finally recombining with the holes bound to the acceptors. Therefore we have clarified the mechanism of energy relaxation by acoustical scattering in the free-to-bound Stokes shifted recombination. Furthermore, we have found that when the exciting energy is below $1.502 \mathrm{eV},(1-\mathrm{LO}+1-\mathrm{LA})$-scattering predominate; when the incident light energy is above $1.502 \mathrm{eV}$, more acoustic phonons may be involved in the impurity scattering so that other higher-order processes like $(1-\mathrm{LO}+(\mathrm{n})-\mathrm{LA})$ $(\mathrm{n}>1)$ scattering should also be taken into account. 
3. Some other spectral features related to hot luminescence and its phonon replicas have been found and discussed. We have observed the low energy "tail" in the PL spectrum and selective pair luminescenceas as well as the up-conversion DAP luminescence. We have interpreted the luminescence "tail" and SPL as due to the impurity spatial distribution effect on the spectra. The many particle effect leads to the line broadening and line shift. By considering deep centers in the GaAs, we have also explained the up-converted luminescence.

At this point, we would like to point out that the distinction between RRS and hot luminescence is not sharp in the case of impurity-induced RRS. For the intrinsic Raman scattering, there exists an explicit distinction which rests upon the fact that the scattering light is "polarized" while the phonon-assisted luminescence is "depolarized". But for the impurity-induced RRS, as shown in this thesis, the selection rules do not hold thus there is no essentially definite polarization for the scattering light. Notice that the impurity states are bound states without a definite $\boldsymbol{k}$, and they are highly degenerate at the same energy level, we then conclude that elastic impurity scattering, or scattering by low energy acoustic phonons, to the degenerate states with different polarization proceeds in the RRS, which makes that the RRS loses the phase memory of the states. So impurity-induced RRS can be regarded as a two- or more- step process and actually can be theoretically treated by using fourth-order perturbation theory Gog76. In such a sense, it has no fundamental difference from the phonon-assisted luminescence, or called luminescence-phonon replicas in the thesis, which may relate to more energy transfer and more steps in the process.

At last, the meaning of the word "extrinsic" in this thesis is relative because even in high-purity GaAs the impurity-induced phenomena are essentially strong (we have also done experiments on other high-purity GaAs samples). This study of impurity-induced scattering gains importance because the impurity scattering may show profound effects at low temperature in other low-impurity semiconductors. 


\section{Appendix A: Parameters for GaAs $(\mathrm{T}=2 \mathrm{~K})$}

\section{Parameters}

Carbon acceptor binding energy

Band gap

Damping constant of hh-exciton

Damping constant of lh-exciton

Damping constant of os-exciton

Deformation potential constant

Donor binding energy

Effective mass of the conduction band

Heavy hole effective mass

Light hole effective mass

LO phonon energy

TO phonon energy

Fröhlich constant

Higher band-band transition energies

Interband momentum matrix-element

Lattice constant

Optical dielectric constant

Reduced mass of primitive cell

Refractive index

Spin-orbit splitting

Static dielectric constant

1-s exciton Bohr radius

1-s exciton bind energy

Symbols
$E_{A}$
$E_{0}$
$\Gamma_{l h}$
$\Gamma_{h h}$
$\Gamma_{o s}$
$d_{0}$
$E_{D}$
$m_{e}$
$m_{h h}$
$m_{l h}$
$\hbar \omega_{L O}$
$\hbar \omega_{T O}$
$\left|c_{F}\right|$
$E_{1}, E_{1}+\Delta_{1}$
$2 p_{c v}^{2} / 3 m$
$a_{0}$
$\epsilon_{\infty}$
$M$
$\eta$
$\Delta_{0}$
$\epsilon_{0}$
$a_{X}$
$E_{X}$

Values

$27 \mathrm{meV}$

$1.520 \mathrm{eV}$

$2.5 \mathrm{meV}$

$2.5 \mathrm{meV}$

$5 \mathrm{meV}$

$31.5 \mathrm{eV}$

$5.8 \mathrm{meV}$

$0.067 \mathrm{~m}$

$0.57 \mathrm{~m}$

$0.082 \mathrm{~m}$

$36.5 \mathrm{meV}$

$33.8 \mathrm{meV}$

$0.14 \mathrm{eV}^{1 / 2}$

$3.028,3.246 \mathrm{eV}$

$13.9 \mathrm{eV}$

$5.65 \AA$

10.6

$6.63 \times 10^{4} \mathrm{~m}$

$\sqrt{\epsilon_{0}}$

$340 \mathrm{meV}$

12.56

$120 \AA$

$4.2 \pm 0.2 \mathrm{meV}$
Reference

Ulb73

Sch72

Can89a

Can89a

Can89a

Tro78,

Sti71

Fet71

Ulb73

Vre68

Iwa64

Iwa64

Can89b

Soo87

Bla82

Bla82

Sti71]

Men85

Men85

Sti71

Can89b

Sel72 


\title{
Appendix B: Symbols and Abbreviations
}

\author{
bcc Body-centered lattice \\ BZ Brillouin zone \\ CB Conduction band \\ CCD Charge-coupled device \\ DAP Donor-acceptor pair(s) \\ DOS Density of states \\ DP Deformation potential (interaction) \\ e-h Electron-hole (pairs) \\ EO Electric-optic (interaction) \\ F Fröhlich (intraband interaction) \\ fcc Face-centered lattice \\ F.H. Faust-Henry (coefficient) \\ FWHM Full width of half maximum \\ hh Heavy hole(s) \\ LA Longitudinal acoustical (phonon) \\ lh Light hole(s) \\ LO Longitudinal optical (phonon) \\ LPB Lower polariton branch \\ O.D. Optical density \\ PC Primitive cell \\ PL Photoluminescence \\ TA Transverse acoustical (phonon) \\ TO Transverse optical (phonon) \\ rhs Right hand side \\ RRS Resonance Raman scattering \\ so Spin-orbit \\ SPL Selective pair luminescence \\ UPB Upper polariton branch \\ VB Valence band
}




\begin{tabular}{|c|c|}
\hline$a_{0}$ & Lattice constant \\
\hline$a_{D P}$ & DP-induced Raman polarizability \\
\hline$a_{F}$ & F-induced Raman polarizability \\
\hline$a_{H}$ & Bohr radius of hydrogen atom \\
\hline$a_{X}$ & Bohr radius of 1-s free exciton \\
\hline$c$ & Speed of light in vacuum \\
\hline$\left|c_{F}\right|$ & Fröhlich constant \\
\hline$c_{q}^{+}, c_{q}$ & Phonon creation and annihilation operators \\
\hline$d_{0}$ & Constant of deformation potential \\
\hline$d_{V A}$ & Volume deformation potential for LA phonons \\
\hline$e$ & Elementary charge \\
\hline$\hat{\mathbf{e}}_{l(s)}, \hat{\mathbf{e}}_{q}$ & $\begin{array}{l}\text { Polarization unit vectors of incident (scattered) light photons and } \\
\text { phonons, respectively }\end{array}$ \\
\hline $\mathrm{e}_{\mathrm{m}}$ & Electromechanical tensor due to piezoelectric interaction \\
\hline$E_{0}$ & Direct band gap energy \\
\hline$E_{1}$ & The higher band-band transition energy around critical point \\
\hline$E_{l}$ & Incident photon energy \\
\hline$E_{s}$ & Scattered photon energy \\
\hline$E_{A}$ & Acceptor binding energy \\
\hline$E_{B X}$ & Bound exciton additional binding energy \\
\hline$E_{D}$ & Donor binding energy \\
\hline$E_{X}$ & Free exciton binding energy \\
\hline$f_{A}$ & Oscillator strength of $\left(e, A^{0}\right)$ \\
\hline$f_{B X}$ & Oscillator strength of bound exciton \\
\hline$f_{c v}$ & Oscillator strength of uncorrelated e-h pairs \\
\hline$f_{X}$ & Oscillator strength of 1-s free exciton \\
\hline$H_{D P}$ & Hamiltonian of the deformation potential \\
\hline$H_{D P-V A}$ & Hamiltonian of the acoustic volume deformation potential \\
\hline$H_{P E}$ & Hamiltonian of the piezoelectric interaction \\
\hline$H_{D P-O}$ & Hamiltonian of the optical deformation potential \\
\hline$H_{E}$ & Electronic Hamiltonian \\
\hline$H_{E R}$ & Hamiltonian of electron-radiation interaction \\
\hline$H_{E P}$ & Hamiltonian of electron-phonon interaction \\
\hline$H_{F}$ & Hamiltonian of the intraband Fröhlich interaction \\
\hline$H_{L}$ & Lattice Hamiltonian \\
\hline $\boldsymbol{k}$ and $k$ & Electron (hole) wave vector and wave number \\
\hline $\boldsymbol{k}_{l(s)}$ & Wave vectors of incident (scattered) photons \\
\hline$M$ & Reduced mass of primitive cell \\
\hline$m, m_{e}, m_{h}$ & $\begin{array}{l}\text { Free electron rest mass, electron effective mass, and hole effective } \\
\text { mass, respectively }\end{array}$ \\
\hline$N$ & Number of unit cells in a crystal \\
\hline$N_{A(D)}$ & Concentration of unfilled acceptors (donors) \\
\hline$N_{A-}$ & Concentration of ionized acceptors \\
\hline
\end{tabular}




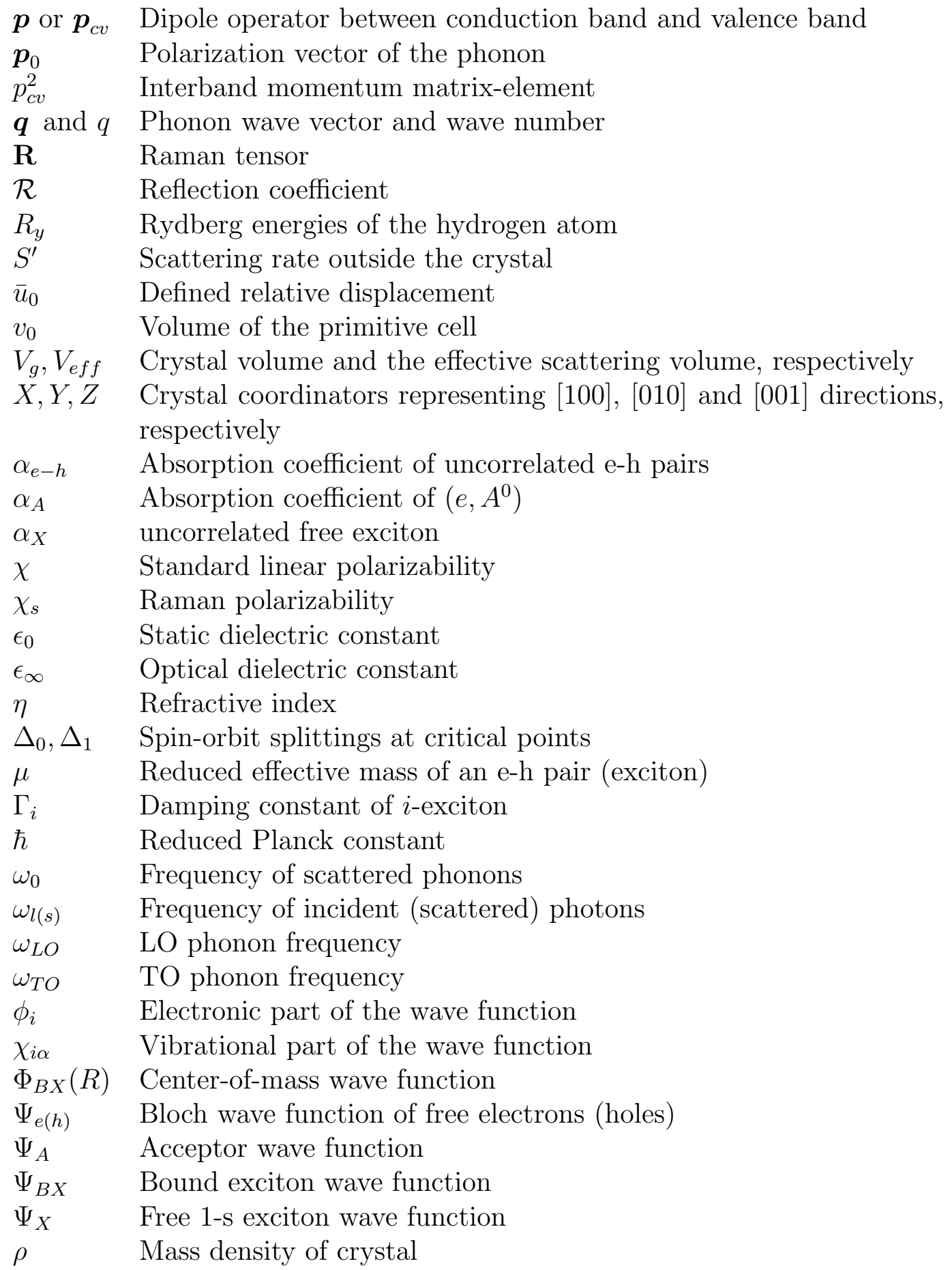

It is valid for all vector $\boldsymbol{c}: c=|\boldsymbol{c}|$ 


\section{References}

[Ada94] S. Adachi, GaAs and Related Materials, World Scientific, Singapore (1994).

[Ale97] V. Alex, T. Iino, J. Weber, EL2-Induced Upconversion Luminescence in GaAs, Mat. Res. Soc. Symp. Proc. 442, 441 (1997).

[Ash75] D. J. Ashen, P. J. Dean, D. T. J. Hurle, J. B. Mullin, A. M. White, and P. D. Greene, The Incorporation and Characterisation of Acceptors in Epitaxial GaAs, J. Phys. Chem. Solids 36, 1041 (1975).

[Asp83] D. E. Aspnes and A. A. Studna, Dielectric Functions and Optical Parameters of Si, Ge, GaP, GaAs, GaSb, InP, InAs, and InSb from 1.5 to $6.0 \mathrm{eV}$, Phys. Rev. B 27, 985 (1983).

[Bec86] S. M. Beck and J. E. Wessel, Observation of Strongly Wave-Vector-Dependent Raman Scattering by LO Phonons in GaAs Near the $E_{0}$ Band Gap, Phys. Rev B 33, 5946 (1986).

[Ber87] R.S. Berg and P.Y. Yu, Resonant Raman Study of Intrinsic Defect Modes in Electron and Neutron-Irradiated GaAs, Phys. Rev. B 35, 2205 (1987).

[Bla82] J. S. Blakemore, Semiconducting and Other Major Properties of Gallium Arsenide, J. Appl. Phys. 53, 123 (1982).

[Bog68] E. H. Bogardus and H. B. Bebb, Bound-Exciton, Free-Exciton, Band-Acceptor, Donor-Acceptor, and Auger Recombination in GaAs, Phys. Rev. 176, 993 (1968).

[Can89a] A. Cantarero, C. Trallero-Giner, and M. Cardona, Excitons in One-Phonon Resonant Raman Scattering: Deformation-Potential Interaction, Phys. Rev. B 39, 8388 (1989).

[Can89b] A. Cantarero, C. Trallero-Giner, and M. Cardona, Excitons in One-Phonon Resonant Raman Scattering: Froehlich and Interference Effects, Phys. Rev. B 40, 12290 (1989).

[CG75-91] Light Scattering in Solids, Topics in Applied Physics, edited by M. Cardona and G. Guentherodt: Vol. 8 (1975), Vol. 50 (1982), Vol. 51 (1982), Vol. 54 (1984), Vol. 66 (1989), Vol. 68 (1991).

[Car82] M. Cardona, Light scattering in Solids, Topics in Applied Physics Vol. 50, edited by M. Cardona and G. Guentherodt, Springer, Heidelberg, pp19-178 (1982). 
[Col70] P. J. Colwell and M. V. Klein, Wave Vector Dependence and Numerical Value of the Scattering Efficiency for the Resonant Raman Effect in CdS, Solid State Commun. 8, 2095 (1970).

[Dam71] T. C. Damen and J. Shah, Bound Exciton Resonance in Raman Cross Sections in CdS, Phys. Rev. Lett. 27, 1506 (1971).

[Das55] W.C. Dash and R. Newman, Intrinsic Optical Absorption in Single-Crystal Germanium and Silicon at $77 \mathrm{~K}$ and $300 \mathrm{~K}$, Phys. Rev. 99, 1151 (1955).

[Dea67] P.J. Dean, W.F. Flood, and G. Kaminsky, Absorption due to Band Exciton in Silicon, Phys. Rev. 163, 721 (1967).

[Dem96] W. Demtroeder, Experimental Physik 3: Atom, Molekuele und Festkoeper, Springer-Verlag, Berlin (1996).

[Dum63] W. P. Dumke, Optical Transitions Involving Impurities in Semiconductors, Phys. Rev. 132, 1998 (1963).

[Eag60] D. M. Eagles, Optical Absorption and Recombination Radiation in Semiconductors due to Transitions between Hydrogen-like Acceptor Impurity Level and the Conduction Band, J. Phys. Chem. Solids 16, 76 (1960).

[Ell57] R. J. Elliott, Intensity of Optical Absorption by Excitons, Phys. Rev. 108, 1384 (1957).

[Fet71] H. R. Fetterman, D. M. Larsen, G. E. Stillman, P. E. Tannenwald, J. Waldman, Phys. Rev. Lett. 26, 975 (1971).

[Fil74] S. P. C. Filho, N. Jannuzzi, E. A. Farah and R. C. C. Leite, Electron-Phonon Coupling in Donor-Acceptor Pair Recombination in CdSe, Solid State Commun. 15, 1749 (1974).

[Fin86] E. Finkman, M. D. Sturge and R. Bhat, Oscillator Strength, Lifetime and Degeneracy or Resonatly Excited Bound Excitons in GaAs, J. Luminescence 35, 235 (1986).

[Gan67] A. K. Ganguly and J. L. Birman, Phys. Rev. 162, 806(1967).

[Gog76] A. A. Gogolin and E. I. Rashba, Mechanism of Strong Resonant 1 LO Raman Scattering, Solid State Commun. 19, 1177 (1976).

[Gri79] M. Grimsditch, D. Olego, and M. Cardona, Absolute Cross-Section of Firstorder Scattering in GaAs, Phys. Rev. B 20, 1758 (1979).

[Gri80] M. Grimsditch and M. Cardona, Absolute Cross-Section for Raman Scattering by Phonons in Silicon, Phys. Stat. Sol. (b) 102, 155 (1980).

[Har90] T. D. Harris, J. K. Trautman and J. I. Colonell, Dynamics of Selectively Excited Donor Acceptor Pairs in GaAs, Materials Science Forum 65-66, 21 (1990).

[Hay78] W. Hayes and R. Loudon, Scattering of Light by Crystals, John-Wiley \& Sons, Inc. (1978). 
[Hei74] U. Heim and P. Hiesinger, Luminescence and Excitation Spectra of Exciton Emission in GaAs, Phys. Stat. Sol. (b) 66, 461 (1974).

[Hwa73] C. J. Hwang, Lifetime of Free and Bound Excitons in High-purity GaAs, Phys. Rev. B 8, 646 (1973).

[Icc99] Abstracts of International Conference on Solid State Spectroscopy (ICSSS), Germany, 1999.

[Iin88] T. Iino, M. Tajima, and K. Ishida, Spectral Shape Analysis of Selective Pair Luminescence in GaAs, J. Appl. Phys. 32, 5454 (1988).

[Iin90] T. Ino, Determination of Residual Donor Concentration by Selective Pair Luminescence in Semi-insulating GaAs, Mat. Sci. Forum, 65-66, 11 (1990).

[Iwa64] S. Iwasa, I. Balslev, and Burstein, Proceedings of the 7th International Conference on Physics of Semiconductors, Paris 1964, edited by M.Hulin, 1077, (1964).

[Joh82] E. J. Johnson, J. Kafalas, R. W. Davis, and W. A. Dyes, Deep Center EL2 and Anti-Stokes Luminescence in Semi-insulating GaAs, Appl. Phys. Lett. 40, 993 (1982).

[Kam76] T. Kamiya and E. Wagner, Shallow Acceptor Binding Energy and Lifetime of Donor-acceptor Pairs in Gallium Arsenide, J. Appl. Phys. 47, 3219 (1976).

[Kan56] E.O. Kane, J. Phys. Chem. Solids 1, 249 (1956).

[Kau89] U.Kaufmann, The Spectroscopic Evidence for the Identity of EL2 and the AsGa Antisite in As-grown GaAs, Festkoeperprobleme 29, 183(1989).

[Kau87a] W. Kauschke, A. K. Sood, M. Cardona, and K. Ploog, Resonance Raman Scattering in GaAs- $\mathrm{Al}_{x} \mathrm{Ga}_{1-x} \mathrm{As}$ Supperlattices: Impurity-Induced FroehlichInteraction Scattering, Phys. Rev. B 36, 1612 (1987).

[Kau87b] W. Kauschke and M. Cardona, Resonant Raman Scattering by LO Phonons near the $E_{0}+\Delta_{0}$ Gap of GaSb, Phys. Rev. B 35, 9619 (1987).

[Kau87c] W. Kauschke, V. Vorlicek, and M. Cardona, Resonant Raman Scattering in GaP: Excitonic and Interference Effects near the $E_{0}$ and $E_{0}+\Delta_{0}$ Gaps, Phys. Rev. B 36, 9129 (1987).

[Kau87d] W. Kauschke, V. Vorlicek, M. Cardona, L. Vina and W.I. Wang, Interference Effects of Raman Scattering by LO-phonons near the $E_{0}+\Delta_{0}$-gap Studied on $(\overline{1} \overline{1} \overline{3}),(111)$, and $(\overline{1} \overline{1} \overline{1})$ Faces of GaAs, Solid State Commun. 61, 487 (1987).

[Kau86] W. Kauschke and M. Cardona, Resonant Raman Scattering and Interference Effects of LO phonons at the $E_{0}+\Delta_{0}$ Gap of InP, Phys. Rev. B 33, 5473 (1986).

[Kis83] D. W. Kisker, H. Tews, and W. Rehm, Luminescence Study of C, Zn, Si, and Ge Acceptors in GaAs, J. Appl. Phys. 54, 1332 (1983).

[Kot79] E. S. Koteles and G. Winterling, Resonant Scattering of Exciton Polaritons by LO and Acoustic Phonons, Phys. Rev. B 20, 628 (1979). 
[Lei67] R.C.C. Leite, Radiative Recombination in n-type InP, Phys. Rev. 157, 672 (1967).

[Lou64] R. Loudon, the Raman Effect in Crystals, Advances in Physics 13, 423 (1964).

[Mac89] M. Maciaszek, D. W. Rogers, and R. P. Bult; and T. Steiner, Y. Zhang, S. Charbonneau, and M.L.W. Thewalt, Photoluminescence for Characterization of Commercial Semi-insulating GaAs, Can. J. Phys. 67, 384 (1989).

[Mal71] H. L. Malm and R. R. Haering, Franck-Condon Effects in the Luminescence of CdS, Canadian J. Phys. 49, 2970 (1971).

[Mar71a] R. M. Martin and C. M. Varma, Cascade Theory of Inelastic Scattering of Light, Phys. Rev. Lett. 26, 1241 (1971).

[Mar71b] R. M. Martin, Phys. Rev. B 4, 3677 (1971).

[Men85] J. Menendez and M. Cardona, Interference Effects: A Key to Understanding Forbidden Raman Scattering by LO Phonons in GaAs, Phys. Rev. B 31, 3696 (1985).

[Mor91] Y. Mori, K. Onozawa, H. Ohkura and Y. Chilba, Anti-Stokes Emission Related to EL2 in GaAs, J. Luminescence 48-49, 88 (1991).

[Oka79] Y. Oka and M. Cardona, Resonance Raman Scattering of Excitonic Polaritons, Solid State Commun. 30, 447 (1979).

[Osb79] G. C. Osbourn and D. L. Smith, Auger and Radiative Transition Rates for Acceptor Bound Excitons in Direct-gap Semiconductors, Phys. Rev. 20, 1556 (1979).

[Pey93] N. Peyghambarian, S. Koch, A. Mysyrowicz, Introduction to Semiconductor Optics, Prentice-Hall, Inc., New Jeysey, 1993.

[Pol66] F. H. Pollak, C. W. Higginbotham, M. Cardona, J.Phys. Soc. Jap. Suppl. 21, 20 (1966).

[Qua84] L. G. Quagliano, H. Nather, Up-conversion of Luminescence via Deep Centers in High-purity GaAs and GaAlAs Epitaxial Layers, Appl. Phys. Lett. 45, 555 (1984).

[Ras62] E. I. Rashba and G. E. Gurgenishvili, Edge Absorption Theory in Semiconductors, Soviet Phys. Solid State 4, 759 (1962).

[Rey93] D. C. Reynolds, D. N. Talwar, M. O. Manasreh, and C. E. Stutz, Phonon Coupling Associated with Free-to-Bound and Bound-to-Bound Transitions in GaAs Layers Grown by Molecular-Beam Epitaxy, Phys. Rev. B 47, 13304 (1993).

[Roe91] T. Roedle, Diplomarbeit: Resonante Lichtstreuung an Galliumarsenid im Bereich des Band-Stoerstellen-Ueberganges, Goettingen (1991) (unpublished).

[Ros70] J. A. Rossi, C. M. Wolfe, and J. O. Dimmock, Acceptor Luminescence in Highpurity n-Type GaAs, Phys. rev. Lett. 25, 1614 (1970). 
[San83] G. D. Sanders and Y. Chang, Radiative Decay of the Bound Exciton in Directgap Semiconductors: the Correlation Effect, Phys. Rev. B 28,5887(1983).

[Sch72] W. Schairer and N. Stath, J. Appl. Phys 43, 447 (1972).

[Seg68] B. Segall and G.D. Mahan, Phonon-Assisted Recombination of Free Excitons in Compound Semiconductors, Phys. Rev. 171, 935 (1968).

[Sel72] D. D. Sell, Resolved Free-exciton Transitions in the Optical-absorption Spectrum of GaAs, Phys. Rev. B 6, 3750 (1972).

[Soo87] A. K. Sood, W. Kauschke, J. Menendez, and M. Cardona, Resonance Raman Scattering by Optical Phonons in GaAs near the $E_{0}$ Band Gap, Phys. Rev. B 35, 2886 (1987).

[Sti71] G. E. Stillman, D. M. Larsen, C. M. Wolfe, and R. C. Brandt, Solid State Commun. 9, 2245 (1971).

[Str90] D. Strauch, B. Dorner, J. Phys.: Condens. Matt. 2, 1457(1990).

[Tew79a] T. Tews, H. Venghaus, and P. J. Dean, Excited States of Shallow Acceptors in ZnSe, Phys. Rev. B 19, 5178 (1979).

[Tew79b] T. Tews and H. Venghaus, Selective Pair Luminescence in Semiconductors, Solid State Commun. 30, 219 (1979).

[Tho64] D. G. Thomas, M. Gershenzon and F. A. Trumbore, Pair Spectra and "Edge" Emission in Gallium Phosphide, Phys. Rev. A 133, 269 (1964).

[Tho65] D. G. Thomas, J. J. Hopfield, and W. M. Augustyniak, Kinetics of Radiative Recombination at Randomly Distributed Donors and Acceptors, Phys. Rev. A 140, 202 (1965).

[Toy70] Y. Toyozawa, Phonon Structures in the Spectra of Solids, J. Luminescence, 1-2, 732 (1970).

[Tra89] C. Trallero-Giner, A. Cantarero, and M. Cardona, One-phonon Resonant Raman Scattering: Froehlich Exciton-phonon Interaction, Phys. Rev. B 40, 4030 (1989).

[Tri88] M. Trinn, Diplomarbeit: Hochaufloesende Absorptionsspectroskopie an Galliumarsenid, Goettingen, 1988 (unpublished).

[Tro78] T. Trommer and M. Cardona, Resonant Raman Scattering in GaAs, Phys. Rev. B 17, 1865 (1978).

[Ulb71] R. Ulbrich, Capture of Hot Electrons by Ionized Donors in GaAs, Phys. Rev. Lett. 27, 1512 (1971).

[Ulb73] R. Ulbrich, Energy Relaxation of Photoexcited Hot Electrons in GaAs, Phys. Rev. B 8, 5719 (1973).

[Ulb78] R. G. Ulbrich, Low Density Photoexcitation Phenomena in Semiconductors: Aspects of Theory and Experiment, Solid-State Electronics 21, 51 (1978). 
[Ulb89] R. G. Ulbrich, Hot-electron Recombination at Neutral Acceptors in GaAs: a CW Probe of Femtosecond Intervalley Scattering, Phy. Rev. Lett. 62, 949 (1989).

[Vin73] A. T. Vink, R. L. A. Van der Heyden and J. A. W. Van der Dose de Bye, The Dielectric Constant of GaP from a Refined Analysis of Donor-Acceptor Pair Luminescence, and the Deviation of the Pair Energy from the Coulomb Law, J. of Luminescence 8, 105 (1973).

[Vre68] Q.H.F. Vrehen, J. Phys. Chem. Solids 29, 129 (1968)

[Wag83] J. Wagner and M. Cardona, Absolute Efficiency and Dispersion of Raman Scattering by Phonons in Silicon, Solid State Commun. 48, 301 (1983).

[Wag86] J. Wagner and M. Ramsteiner, Residual Acceptor Assessment in As-Grown Bulk GaAs by Raman and Selective Pair Luminescence Spectroscopiy: A Comparative Study, Appl. Phys. Lett. 49, 1369 (1986).

[Wag87] J. Wagner and M. Ramsteiner, Binding Energies of Shallow Donors in Semiinsulating GaAs, J. Appl. Phys. 62, 2148 (1987).

[Wau63] J.L.T. Waugh G. Dolling, Phys. Rev. 132, 2410 (1963).

[Whi74] A. M. White, D. J. Dean, and B. Day, On the Origin of Bound Exciton Lines in Indium Phosphide and Gallium Arsenide, J. Phys. C 7, 1401 (1974).

[Wil70] M.L. Williams and J. Smit, Multiple-Phonon Resonant Raman Scattering Theory, Solid State Commun. 8, 2009 (1970).

[Yu75] P. Y. Yu and Y. R. Shen, Resonance Raman Studies in $\mathrm{Cu}_{2}$ O. I: The Phononassisted 1s Yellow Excitonic Absorption Edge, Phys. Rev. B 12, 1377 (1975).

[Yu99] P. Y. Yu, M. Cardona, Fundamentals of Semiconductors: Physics and Materials Properties (second edition), Springer Verlag, Heidelberg (1999).

[Zey75] R.Zeyher, Theory of Multiphonon Raman Spectra above the Energy Gap in Semiconductors, Solid State Commun. 16, 49 (1975). 


\section{Acknowledgment}

Here I wish to express my sincere thanks to every person who has ever helped me with this work in the past.

At first I would like to record my indebtedness to Professor Dr. R. G. Ulbrich. His continuos support, pertinent advice and valuable criticism are greatly appreciated for the essential progress of my study and research carried out in Germany. It is him who has introduced me to the semiconductor science and brought me to the insight into many aspects in solid state physics. I also acknowledge with thanks the hospitality offered by him when I came to Germany as a "VW-Stipentiat".

It is my pleasant duty to thank Professor Dr. W. Felsch for his kind readiness to appraise this work.

Gratitude also goes to my many colleagues in the IV. Physics Institute. Before my starting the research, many German researchers and students have helped me to get familar with the institute: the laboratories, the instruments, and the personnel. These people merit special thanks: T. Rödle for introducing and handing over the Lab. of Raman Spectroscopy to me, and S. Piezonka, J. Glander, and K. Engel for the helpful collabration in my study of Au-Si interface under annealing condition. For my thesis work, although I have been the unique person in the Lab. of Raman Spectroscopy for the last three years, I benefited from some technical assistance from P. Blome. I also wish to thank Dr. M. Hübner, Dr. M. Wenderoth and many others for their interest in my work as shown in both group discussions and personal talks.

I am also indebted to all those persons who can promptly grant the help in many administration or routine matters: Mr. Sonnenberg for the help with mechanical instruments, Mr. Langohr for the help with electrical apparatus, Ms. Edith Rohrmoser and Dr. H. D. Schulte for administration facilities.

The financial support from Volkswagen-Stiftung is gratefully acknowledged.

Last but not least, I would like to express the gratitude towards my parents S. B. Huang and X. S. Gu, and my wife Q. Guo: their encouragement and understanding have sustained me through many difficulties and challenging moments during my study abroad. 
